\title{
A Far Ultraviolet Spectroscopic Explorer Survey of Interstellar Molecular Hydrogen in the Galactic Disk
}

\author{
J. Michael Shull, Charles W. Danforth \\ Department of Astrophysical and Planetary Sciences and CASA \\ University of Colorado, 389-UCB, Boulder, CO 80309 \\ Katherine L. Anderson \\ Green Mountain High School, Lakewood, CO 80228, USA \\ michael.shull@colorado.edu, charlesdanforth@gmail.com, \\ kalander@jeffcoschools.us
}

\begin{abstract}
We report results from a Far Ultraviolet Spectrographic Explorer (FUSE) survey of interstellar molecular hydrogen $\left(\mathrm{H}_{2}\right)$ in the Galactic disk toward 139 O-type and early B-type stars at Galactic latitudes $|b| \leq 10^{\circ}$, with updated photometric and parallax distances. The $\mathrm{H}_{2}$ absorption is measured using the far-ultraviolet Lyman and Werner bands, including strong $\mathrm{R}(0), \mathrm{R}(1)$, and $\mathrm{P}(1)$ lines from rotational levels $J=0$ and $J=1$ and excited states up to $J=5$ (sometimes $J=6$ and 7). For each sight line, we report column densities $N_{\mathrm{H} 2}, N_{\mathrm{HI}}, N(J), N_{\mathrm{H}}=N_{\mathrm{HI}}+2 N_{\mathrm{H} 2}$, and molecular fraction, $f_{\mathrm{H} 2}=2 N_{\mathrm{H} 2} / N_{\mathrm{H}}$. Our survey extends the 1977 Copernicus $\mathrm{H}_{2}$ survey up to $N_{\mathrm{H}} \approx 5 \times 10^{21} \mathrm{~cm}^{-2}$. The lowest rotational states have excitation temperatures and rms dispersions, $\left\langle T_{01}\right\rangle=88 \pm 20 \mathrm{~K}$ and $\left\langle T_{02}\right\rangle=77 \pm 18 \mathrm{~K}$, suggesting that $J=0,1,2$ are coupled to the gas kinetic temperature. Populations of higher- $J$ states exhibit mean excitation temperatures, $\left\langle T_{24}\right\rangle=237 \pm 91 \mathrm{~K}$ and $\left\langle T_{35}\right\rangle=304 \pm 108 \mathrm{~K}$, produced primarily by UV radiative pumping. Correlations of $f_{\mathrm{H} 2}$ with $E(B-V)$ and $N_{\mathrm{H}}$ show a transition to $f_{\mathrm{H} 2} \geq 0.1$ at $N_{\mathrm{H}} \gtrsim 10^{21} \mathrm{~cm}^{-2}$ and $E(B-V) \gtrsim 0.2$, interpreted with an analytic model of $\mathrm{H}_{2}$ formation-dissociation equilibrium and attenuation of the far-UV radiation field by self-shielding and dust opacity. Results of this disk survey are compared to previous FUSE studies of $\mathrm{H}_{2}$ in translucent clouds, at high Galactic latitudes, and in the Magellanic Clouds. Using updated distances to the target stars, we find average sightline values $\left\langle f_{\mathrm{H} 2}\right\rangle=0.20$ and $\left\langle N_{\mathrm{H}} / E(B-V)\right\rangle=6.07 \times 10^{21} \mathrm{~cm}^{-2} \mathrm{mag}^{-1}$.
\end{abstract}

\section{Introduction}

Molecular hydrogen $\left(\mathrm{H}_{2}\right)$ is the most abundant molecule in the universe, constituting the majority of the interstellar molecular clouds that eventually form stars. Even though $\mathrm{H}_{2}$ plays an important role in the chemistry of the interstellar medium (ISM), many questions remain about its distribution, formation, and destruction in both diffuse and protostellar clouds. The Copernicus satellite in the 1970s provided the first large-scale survey of interstellar $\mathrm{H}_{2}$ (Spitzer et al. 1974; Savage et al. 1977). With the 1999 launch of the Far Ultraviolet Spectrographic Explorer (FUSE) satellite, astronomers once again gained access to the far-ultraviolet (FUV) wavelengths needed to study $\mathrm{H}_{2}$ in its resonance absorption lines. This survey extends these studies to 139 OB-type stars, many of them fainter and more distant than observed by Copernicus.

From the inception of the FUSE mission, $\mathrm{H}_{2}$ studies were part of the science plan. The FUSE satellite, its mission, and its on-orbit performance were described in Moos et al. (2000) and Sahnow et al. (2000). 
Initial FUSE studies of $\mathrm{H}_{2}$ were reported in papers from the Early Release Observations (Snow et al. 2000; Shull et al. 2000). Later studies included a survey of 70 sight lines to $\mathrm{OB}$ stars probing $\mathrm{H}_{2}$ in the Large and Small Magellanic Clouds (Tumlinson et al. 2002), surveys of 38 sight lines through translucent clouds (Rachford et al. 2002, 2009), studies of chemical relationships of $\mathrm{H}_{2}$ with other molecules $\left(\mathrm{CO}, \mathrm{CH} \mathrm{CH}^{+}\right.$, $\mathrm{CN}$ ) and atomic species (Burgh et al. 2007; Sheffer et al. 2008; Jensen et al. 2010), and observations of $\mathrm{H}_{2}$ in the low Galactic halo (Gillmon et al. 2006; Wakker 2006; Gillmon \& Shull 2006). At high redshift, $\mathrm{H}_{2}$ Lyman/Werner lines have been detected in damped Ly $\alpha$ absorbers (Noterdaeme et al. 2008; Jorgenson et al. 2014; Balashev et al. 2019).

We present the results of a FUSE survey of interstellar $\mathrm{H}_{2}$ absorption in the Galactic disk, using transitions from the ground electronic state, $X^{1} \Sigma_{g}^{+}$, to excited electronic states, $B^{1} \Sigma_{u}^{+}$(Lyman bands) and $C^{1} \Pi_{u}$ (Werner bands). These lines are rovibrational transitions from lower states $\left(v_{l}, J_{l}\right)$ to upper states $\left(v_{u}, J_{u}\right)$. In the cold, low-density ISM, essentially all the molecules are in the ground vibrational state $\left(v_{l}=0\right)$. Absorption lines were observed up to $J_{l}=5-6$ and occasionally $J_{l}=7$. Because the wavefunction for the homonuclear $\mathrm{H}_{2}$ is anti-symmetric under interchange of the identical (fermionic) protons, the evenparity rotational states $(J=0,2,4 \ldots)$ have total nuclear spin $S=0$ (spin anti-symmetric para- $\left.\mathrm{H}_{2}\right)$ while odd-parity states $(J=1,3,5 \ldots)$ have $S=1$ (spin-symmetric ortho- $\mathrm{H}_{2}$ ). The statistical weights of these states are $g_{J}=(2 S+1)(2 J+1)$, with odd- $J$ states having a factor of three higher weight. Absorption bands leading to the upper level are identified by changes in vibrational state $\left(v_{u}-v_{l}\right)$ and rotational angular momentum $\left(J_{u}-J_{l}\right)$. The upper electronic state $\left({ }^{1} \Sigma_{u}^{+}\right)$of the Lyman bands has angular momentum $\Lambda=0$ along the internuclear axis. Dipole-allowed changes in rotational state $(\Delta J= \pm 1)$ are denoted as R-branch $\left(J_{u}=J_{l}+1\right)$ and P-branch $\left(J_{u}=J_{l}-1\right)$. The upper electronic state $\left({ }^{1} \Pi_{u}\right)$ of Werner bands has $\Lambda=1$, allowing a Q-branch $\left(J_{u}=J_{l}\right)$ in addition to the $\mathrm{R}$ and $\mathrm{P}$ branches.

We observed multiple lines in the Lyman and Werner bands in the FUV (930-1126 ^) towards background OB stars located near the Galactic disk plane at $|b| \leq 10^{\circ}$. Several target stars were outside this latitude range, but all show strong $\mathrm{H}_{2}$ absorption. Lines from $J=0$ and $J=1$ nearly always exhibit damping wings. Analysis of absorption-line equivalent widths and damping wings yields column densities, $N(J)$, in individual rotational states. Figure 1 shows a FUSE spectrum of the sight line to HD 46150, an O5 Vf star at $1.5 \mathrm{kpc}$ distance and $E(B-V)=0.45$, with the Lyman and Werner bands labeled including a close-up of the (4-0) Lyman band. The total $\mathrm{H}_{2}$ column density, $N_{\mathrm{H} 2}$, is found by summing over all observed $J$ states. Typically, $98 \%$ to $99 \%$ of the molecules reside in the lowest two levels, $J=0$ and $J=1$.

Observations of $\mathrm{H}_{2}$ column densities, the molecular fraction in diffuse clouds, and its rotational excitation provide diagnostics of diffuse ISM (Shull \& Beckwith 1982). The excitation temperature, $T_{01}$, of the lowest rotational states (para- $\mathrm{H}_{2}$ in $J=0$ and ortho- $\mathrm{H}_{2}$ in $J=1$ ) is an approximate measure of the gas kinetic temperature. In many cases, the three lowest levels $(J=0,1,2)$ appear to be thermalized, with both $T_{01}$ and $T_{02}$ coupled to the kinetic temperature (Gry et al. 2002; Le Petit et al. 2006). In diffuse interstellar clouds, thermal equilibration requires sufficiently large gas densities for proton interchange collisions to produce ortho-para conversion between $J=0$ and $J=1$ (Dalgarno et al. 1973; Gerlich 1990). Column densities $N_{\mathrm{H} 2} \geq 10^{18} \mathrm{~cm}^{-2}$ are needed to produce strong self-shielding in absorption lines from the dissociating FUV radiation. The current observations show that both $T_{01}$ and $T_{02}$ provide estimates of the heating and cooling processes in the diffuse ISM. The excitation temperature, $T_{\text {exc }}$, of higher rotational states $(J=3-6)$ is influenced by the FUV radiation field, which excites and photodissociates $\mathrm{H}_{2}$ (Jura 1974; Black \& Dalgarno 1976). From FUSE data on these excited states, we compute temperatures, $T_{24}$ and $T_{35}$, based on population ratios of $J=2$ to 4 and $J=3$ to 5 , respectively.

Section 2 begins with a description of the OB-star sample and our methods of data acquisition and 
analysis. Section 3 provides the survey results, including $\mathrm{H}_{2}$ and $\mathrm{H}$ I column densities, molecular fractions, rotational excitation temperatures, and gas-to-dust ratio, $N_{\mathrm{H}} / E(B-V)$. We also present a simple analytic model of the transition from $\mathrm{H}$ I to $\mathrm{H}_{2}$, including $\mathrm{H}_{2}$ formation rates, FUV dissociation, self-shielding, and dust opacity. The transition occurs at $f_{\mathrm{H} 2} \approx 0.1$ at dust optical depth $\tau_{d} \approx 1$ and is controlled primarily by the ratio of FUV flux to gas density. The lowest rotational states exhibit mean excitation temperatures $\left\langle T_{01}\right\rangle=88 \pm 20 \mathrm{~K}$ and $\left\langle T_{02}\right\rangle=77 \pm 18 \mathrm{~K}$. Higher rotational states $(J \geq 3)$ have larger excitation temperatures, $T_{\text {exc }} \approx 150-650 \mathrm{~K}$, arising from fluorescent cascade following FUV radiative excitation in the Lyman and Werner bands. Section 4 summarizes our findings, with comparisons to the Copernicus survey of $\mathrm{H}_{2}$ (Savage et al. 1977) and the $N_{\mathrm{H}} / E(B-V)$ ratio (Bohlin et al. 1978). The FUSE mean value for the Galactic disk, $\left\langle N_{\mathrm{H}} / E(B-V)\right\rangle=(6.07 \pm 1.01) \times 10^{21} \mathrm{~cm}^{-2} \mathrm{mag}^{-1}$, is lower than estimates from 21-cm/far-IR studies at high Galactic latitudes (Liszt 2014a,b), suggesting that gas and dust have different spatial distributions above the disk.

\section{Data Acquisition and Reduction}

\subsection{FUSE Observations}

The 139 targets in this survey were drawn primarily from FUSE programs designed to study OB stars and interstellar gas in the Milky Way. Figure 2 shows the target distribution in Galactic longitude and latitude, with color coding for their distances. Most stars are O-type and early B-type (B0, B0.5) and generally more distant than those in the 1977 Copernicus survey. Many were part of FUSE science team projects to study O VI from hot gas in the Galactic disk (Bowen et al. 2008), interstellar D/H (Moos et al. 2002; Hoopes et al. 2003; Hébrard et al. 2005), and hot stars and their winds (Massa et al. 2003). Several stars were analyzed specifically for their interstellar $\mathrm{H}_{2}$ (Shull et al. 2000; Snow et al. 2000).

Table 1 lists information on the 139 stellar targets and their observational characteristics. These stars have updated spectral types (SpT) and both photometric and Gaia-DR2 parallax distances determined by Shull \& Danforth (2019) using new information from the Galactic O-star Spectroscopic Survey (Maíz Apellániz et al. 2004). The GOS project generated a large sample of O stars within several kiloparsecs of the Sun with updated spectral classification (Sota et al. 2011, 2014), together with digital photometry and optical-NIR dust extinction (Maíz-Apellániz \& Barbá 2018). The first ten columns of Table 1 provide our internal target ID, star name, Galactic coordinates $(\ell, b)$, photometry ( $B$ and $V$ magnitudes), color excess $E(B-V), \mathrm{SpT}$, and both photometric and parallax distances. The last two columns list the FUSE program ID and exposure time of the primary observation. In some cases, we used other FUSE observations to supplement or confirm our measurements. Footnotes explain the sources for photometry and distances.

All column densities are expressed in units of $\mathrm{cm}^{-2}$ and often quoted in logarithmic format $(\log N)$. The total hydrogen column density, $N_{\mathrm{H}}=N_{\mathrm{HI}}+2 N_{\mathrm{H} 2}$, is used to derive the molecular fraction, $f_{\mathrm{H} 2}=2 N_{\mathrm{H} 2} / N_{\mathrm{H}}$, and explore its correlations with $E(B-V)$ and line-of-sight pathlength. Most $\mathrm{H}$ I column densities, $N_{\mathrm{HI}}$, were taken from previous Ly $\alpha$ profile-fitting surveys (Shull \& Van Steenberg 1985; Diplas \& Savage 1994; Jenkins 2019) supplemented by a few individual Ly $\alpha$ measurements. Table 2 presents our adopted values of $N_{\mathrm{HI}}$ and a comparison to previous measurements. We adopted $N_{\mathrm{HI}}$ in priority order of: (J19) 57 stars from Jenkins (2019); (DS94) 51 stars from Diplas \& Savage (1994); (FM90) two stars from Fitzpatrick \& Massa (1990); and three stars from other sources. A comparison between J19 and DS94 found generally good agreement, within $0.03-0.08$ in $\log N$. Only seven sight lines differed by larger amounts $(0.09-0.19)$. For 26 sight lines with no available no Ly $\alpha$ fits, we estimated $N_{\mathrm{HI}}$ from the scaling relation (Bohlin et al. 
1978) of total hydrogen column density with color excess, $N_{\mathrm{H}}=\left(5.8 \times 10^{21} \mathrm{~cm}^{-2} \mathrm{mag}^{-1}\right) E(B-V)$, where $N_{\mathrm{HI}}=N_{\mathrm{H}}-2 N_{\mathrm{H} 2}$.

\subsection{Data Analysis}

The data analysis for this survey used numerous absorption lines from $\mathrm{H}_{2}$ Lyman and Werner transitions between 930 - $1126 \AA$. Our methodology is identical to that used in the FUSE survey of $\mathrm{H}_{2}$ in the LMC and SMC (Tumlinson et al. 2002), and we refer readers to that paper for details. We search for absorption lines from $J=0-7$ and typically detect lines up to $J=5$. Most lines exhibit shifts in wavelength $(0.03-0.13 \AA)$ with offsets determined by measuring narrow interstellar metal lines such as Ar I(1048.218, $1066.660 \AA)$, Fe II (1055.269 $\AA, 1063.177 \AA, 1144.938 \AA)$, Si II (1020.699 $\AA)$, P II (1152.818 $\AA$ ), and other lines of Fe II, O I, N I. This procedure is similar to that employed by Tumlinson et al. (2002), Wakker (2006), and Gillmon et al. (2006). We also considered the R(0) lines of HD in its (3-0), (4-0), (5-0) bands at 1066.271 $\AA, 1054.286 \AA$ and $1042.847 \AA$, respectively. However, the HD lines were often offset in velocity from $\mathrm{H}_{2}$, possibly because of its presence in only one of the components of the $\mathrm{H}_{2}$ line profile.

The most accurate determinations of column densities $N$ come from weak lines where equivalent width $W_{\lambda} \propto N$, or from strong lines with damping wings where $W_{\lambda} \propto N^{1 / 2}$. In both limits, the column density can be determined without knowledge of the Doppler parameter $(b)$. Between these two regimes, from line-center optical depth $\tau_{0} \approx 1$ up to $\tau_{\text {damp }} \approx 10^{3-4}$, one must employ curve-of-growth (CoG) methods $\rrbracket^{1}$ that require an estimation of $b$. The linear relation between $W_{\lambda}$ and $N$ and the onset of line saturation are measured by optical depth at line center,

$$
\begin{aligned}
W_{\lambda} & =\left(\frac{\pi e^{2}}{m_{e} c}\right) \frac{N f \lambda^{2}}{c}=(88.53 \AA) N_{15} \lambda_{1000}\left[\frac{f \lambda}{10 \AA}\right] \\
\tau_{0} & =\left(\frac{\pi e^{2}}{m_{e} c}\right) \frac{N f \lambda}{\sqrt{\pi} b}=(1.497) N_{15} b_{10}^{-1}\left[\frac{f \lambda}{10 \AA}\right] .
\end{aligned}
$$

Here, we scaled to column density $N=\left(10^{15} \mathrm{~cm}^{-2}\right) N_{15}$, Doppler parameter $b=\left(10 \mathrm{~km} \mathrm{~s}^{-1}\right) b_{10}$, and wavelength $\lambda=(1000 \AA) \lambda_{1000}$. Line strengths are normalized to $f \lambda=10 \AA$, a typical value for many lines in the Lyman and Werner bands.

Equivalent widths of the highly saturated lines from $J=2$ and $J=3$ are frequently quite large, with dimensionless values $W_{\lambda} / \lambda \approx(1-3) \times 10^{-4}$. On the flat portion of the CoG, these widths are primarily determined by the effective $b$-value, with asymptotic values $W_{\lambda} / \lambda \approx(2 b / c)\left[\ln \left(\tau_{0} / \ln 2\right)\right]^{1 / 2}$. As shown in the CoG suite prepared by McCandliss (2003), strong lines on the equivalent width plateau often require Doppler parameters, $b=10-20 \mathrm{~km} \mathrm{~s}^{-1}$, much higher than the expected thermal values, $b_{\mathrm{th}}=(2 \mathrm{kT} / \mathrm{m})^{1 / 2} \approx$ $\left(0.9 \mathrm{~km} \mathrm{~s}^{-1}\right) T_{100}^{1 / 2}$, for $\mathrm{H}_{2}$ at temperatures $T=(100 \mathrm{~K}) T_{100}$. They likely result from velocity components within the absorber and micro-turbulence. Several previous studies (Jenkins \& Peimbert 1997; Gry et al. 2002; Lacour et al. 2005) suggested that $b$-values increase with rotation level. Although we found several

\footnotetext{
${ }^{1}$ The equivalent width $\left(W_{\lambda}\right)$ of a saturated absorption line rises slowly with increasing column density $N$ and line-center optical depth $\tau_{0}$. As shown by asymptotic analysis, $W_{\lambda} / \lambda \approx(2 b / c)\left[\ln \left(\tau_{0} / \ln 2\right)\right]^{1 / 2}$ for large $\tau_{0}$ and a Doppler-broadened Gaussian velocity profile, $\phi(v) \propto \exp \left(-v^{2} / b^{2}\right)$. Draine (2011) noted that this formula is accurate to $5 \%$ for $1.254<\tau_{0}<\tau_{\text {damp }}$, up to the onset of damping wings. Inverting this relation, we find $\tau_{0} \approx(0.693) \exp \left[\left(W_{\lambda} / \lambda\right)^{2} /(2 b / c)^{2}\right]$. Clearly, $\tau_{0}$ and $N$ are exponentially sensitive to measurements of $W_{\lambda}$ and $b$. When equivalent widths of the accessible lines lie on a flat CoG, errors can exceed \pm 0.40 for $\log N=16-17$ and \pm 0.50 for $\log N=17-19$.
} 
cases where the higher- $J$ lines required larger $b$-values, we saw no evidence of a uniform trend in the current survey or in our study of $\mathrm{H}_{2}$ in the Magellanic Clouds (Tumlinson et al. 2002).

For absorbers with $N(J) \leq 10^{18} \mathrm{~cm}^{-2}$, we measured equivalent widths of the accessible $\mathrm{H}_{2}$ lines, producing a CoG that yields a $b$ value and column densities, $N(J)$, in rotational states $J=0-5$, and occasionally $J=6$ and $J=7$. Table 3 lists column densities in $J=0-6$ and the inferred $b$-value when measurable. Most column densities range from $\log N(J) \approx 19.0-21.5$ in $J=0-1$ to $\log N(J) \approx 13.7-15.5$ in $J=4-6$. In 48 targets, we found detectable column densities in $J=6$. For other sight lines, we quote upper limits, which range from $\log N(6)<13.85-14.40$ depending on the data quality. Six targets had detectable column densities in $J=7$, described in footnote (a) to Table 3.

For absorbers with damping wings in $\mathrm{R}(0), \mathrm{R}(1)$, and $\mathrm{P}(1)$ lines, we fitted line profiles to derive $N(0)$ and $N(1)$, a technique used in previous studies of $\mathrm{H}_{2}$ (Savage et al. 1977; Tumlinson et al. 2002; Jenkins 2019). The errors on these column densities depend on data quality, both the signal-to-noise $(\mathrm{S} / \mathrm{N})$ and our ability to define the continuum on either side of the blended $R(0), R(1), P(1)$ complex (see the bottom panel of Figure 1 for an example). Based on our experience with fitting damping wings, we define three levels of $\mathrm{S} / \mathrm{N}$ ratio and corresponding errors, $\sigma_{\log N}$, on $\log N(0)$ and $\log N(1):(1) S / N \geq 15$ (0.03-0.05 errors); (2) $5 \leq S / N \leq 15(0.05-0.10)$; and $(3) S / N \leq 5(0.10-0.20)$.

A key aspect of our analysis software is the rapid and consistent measurement of as many individual $\mathrm{H}_{2}$ absorption lines as possible in each sight line. We then use CoGs to derive column densities for the undamped lines in $J \geq 2$. The programs are written to consistently measure all available (unblocked) absorption lines in the spectra. In many instances, lines from $J=2$ and $J=3$ were strongly saturated, with equivalent widths lying on the flat (Doppler-broadened) portion of the CoG where column densities are difficult to determine. In this case, $W_{\lambda}$ depends primarily on the Doppler parameter, which is often much larger than the thermal value because of multiple velocity components. The process of measuring the numerous lines that enter the CoG fitting cannot be automated completely. Our software requires the user to decide on a line-by-line basis which $\mathrm{H}_{2}$ lines will be fitted. We ignore $\mathrm{H}_{2}$ lines in spectral regions near strong interstellar absorption or bright geocoronal emission. For example, the $\mathrm{H}_{2}$ Lyman (6-0) band is obscured by strong damping wings of the interstellar Ly $\beta$ line (1025.722 $\AA$ ), and the Lyman (5-0) band lies among the resonance absorption lines of C II (1036.337 $\AA)$ and C II* (1037.018 $\AA)$. We neglect these bands except when the higher- $J$ lines are separated from the intervening absorption.

For the higher- $J$ lines, the software steps through the expected positions of the lines, band by band, shifted by the approximate velocity offset from the metal lines. Going through each complete band of lines consecutively, the routine displays the area where a line should appear. If the line is present and unblended with any other transitional or metal line, it is fitted to a Gaussian, and its equivalent width, errors, wavelength, full width half maximum, and velocity offset are entered into a table. The Lyman (6-0) band is typically omitted, and the Lyman (5-0) band is measured only partially, because of overlap with C II $\lambda 1036$ absorption. In these bands, lines that are observable and undistorted are measured and included in the tables. This routine is executed for each detector segment, creating four tables of data, with maximum redundancy over the critical range between $1000 \AA$ and $1126 \AA$. The four tables are then merged, and a CoG is generated with a single Doppler parameter. An error routine is run to calculate the smallest errors over a range of $b$-values. Asymmetric error bars are generated for $b$ and column densities.

As noted above, column densities from $J=2$ and $J=3$ are difficult to measure, because most of their lines are highly saturated. To alleviate CoG uncertainties and determine an accurate $b$-value it is helpful to measure the weakest available lines, with strengths $f \lambda \leq 4 \AA$. These weak lines are useful in anchoring the 
CoG for more saturated lines. Unfortunately, we were unable to measure the weakest lines from $J=2$ : $(0-0)$ $\mathrm{P}(2)$ at $1112.495 \AA(f \lambda=0.740 \AA) ;(0-0) \mathrm{R}(2)$ at $1110.120 \AA(f \lambda=1.199 \AA)$; and $(1-0) \mathrm{R}(2) 1094.244 \AA$ $(f \lambda=4.016 \AA)$. These lines are blended with (0-0) $\mathrm{P}(1),(0-0) \mathrm{R}(3)$, and (1-0) $\mathrm{P}(1)$, respectively. For $J=3$, we were unable to measure the two weakest lines: $(0-0) \mathrm{P}(3)$ at $1115.896 \AA(f \lambda=0.784 \AA)$ blended with $(0-0)$ $\mathrm{R}(4)$ at $1116.013 \AA$, and $(0-0) \mathrm{R}(3)$ at $1112.584 \AA(f \lambda=1.135 \AA)$ blended with $(0-0) \mathrm{P}(2)$ at $1112.495 \AA$. The weakest line that could be measured was (1-0) $\mathrm{P}(3)$ at $1099.788 \AA(f \lambda=2.639 \AA)$. For $J=4$, the weakest lines, $(0-0) \mathrm{P}(4)$ at $1120.247 \AA(f \lambda=0.808 \AA)$ and $(0-0) \mathrm{R}(4)$ at $1116.013 \AA(f \lambda=1.116 \AA)$, are blended with (0-0) $\mathrm{P}(3) 1115.896 \AA$ and $(0-0) \mathrm{R}(5) 1120.300 \AA$, respectively. In some cases we were able to separate (0-0) $\mathrm{P}(4)$. In general the most accessible lines were the $\mathrm{P}(4)$ lines in (1-0), (2-0), (3-0), (4-0), (5-0), (7-0) bands and the R(4) lines in (3-0), (4-0), (5-0) bands. For $J=5$, we found a sufficient number of lines with a range of strengths for a reliable CoG.

A systematic uncertainty in our results comes from the possibility of multiple components in the absorption lines caused by more than one cloud in the line of sight. Many spectra $(\sim 40$ sight lines $)$ have velocity components that make the neighboring lines visually identifiable, but not separable without careful profile fitting using other information from higher resolution optical lines. Some absorbers in this survey have components that are not resolvable; those lines are treated as though they are a single component. The spectra of 15 targets exhibited obvious multiple components separated by $\Delta v \geq 20 \mathrm{~km} \mathrm{~s}^{-1}$. We measured those individually using a double Voigt profile fit. The components have their values entered into separate data tables, enabling us to generate two CoGs with individual $b$ values. In these cases, we report the total column densities measured for saturated lines or lines without visible structure and individual column densities where components are measurable. Lines at $J \leq 3$ are often saturated and too strong to show component structure. These lines are fitted as a single component, and a total column density is returned.

\section{Results}

\subsection{Molecular Abundances}

The FUSE survey finds $\mathrm{H}_{2}$ everywhere in the disk of the galaxy (Figure 2) at typical Galactic latitudes $|b|<4^{\circ}$. The $\mathrm{H}_{2}$ column density rises rapidly above $N_{\mathrm{H} 2} \geq 10^{19.5} \mathrm{~cm}^{-2}$ for sight lines with color excess $E(B-V) \gtrsim 0.2$, as illustrated in Figure 3. The molecular fraction, $f_{\mathrm{H} 2}=2 N_{\mathrm{H} 2} / N_{\mathrm{H}}$, quantifies the number of hydrogen nuclei bound into $\mathrm{H}_{2}$ molecules, where $N_{\mathrm{H}}=N_{\mathrm{HI}}+2 N_{\mathrm{H} 2}$ is the total hydrogen column density. Later in this section, we will adopt $N_{\mathrm{H} 2}=10^{19.5} \mathrm{~cm}^{-2}$ and $f_{\mathrm{H} 2}=0.1$ as nominal values of the atomic-to-molecular transition in the absorbing gas. With the exception of sight lines toward HD 3827 $\left(\log N_{\mathrm{H} 2}=17.48\right), \mathrm{HD} 201638\left(\log N_{\mathrm{H} 2}=18.23\right)$, and HD $92554\left(\log N_{\mathrm{H} 2}=18.93\right)$, all targets in the survey have $\log N_{\mathrm{H} 2} \geq 19.0$. Table 4 summarizes the column densities $\left(N_{\mathrm{HI}}, N_{\mathrm{H} 2}, N_{\mathrm{H}}\right)$ together with the molecular fractions and rotational excitation temperatures inferred from populations in levels $J=0-5$. Rotational excitation temperatures, $T_{01}, T_{02}, T_{24}$, and $T_{35}$, are discussed further in Sections 3.4 and 3.5.

The atomic-to-molecular transition arises from both $\mathrm{H}_{2}$ self-shielding in optical thick lines and dust attenuation of the dissociating FUV radiation (Browning et al. 2003; Krumholz et al. 2008, 2009; Sternberg et al. 2014). The Copernicus survey of 61 stellar targets (Savage et al. 1977) noted that $f_{\mathrm{H} 2}$ rises rapidly above $1 \%$ at $E(B-V) \geq 0.08$ and $N_{\mathrm{H}} \geq 5 \times 10^{20} \mathrm{~cm}^{-2}$. A transition to $f_{\mathrm{H} 2}=0.1$ appeared at lower hydrogen column densities ( $\left.\log N_{\mathrm{H}} \approx 20.4\right)$ in sight lines at high Galactic latitude (Gillmon et al. 2006). In the lower-metallilcity LMC and SMC (Tumlinson et al. 2002; Browning et al. 2003) the transition shifted to higher column densities $\left(\log N_{\mathrm{H}} \approx 21.3-22.0\right.$ ), consistent with models with lower metallicity but a larger 
ratio of FUV radiation to gas density. A lower grain abundance, owing to fewer refractory heavy elements, will reduce the $\mathrm{H}_{2}$ formation rate. It also allows deeper penetration of FUV photons into the cloud, resulting in more $\mathrm{H}_{2}$ dissociation. These effects are discussed further in Section 3.3.

Figure 4 presents the distributions of molecular fraction vs. $E(B-V)$ and $N_{\mathrm{H}}$ for 139 targets in the FUSE survey. Figure 5 plots mean values of $f_{\mathrm{H} 2}$ and hydrogen density $n_{\mathrm{H}}=N_{\mathrm{H}} / D$ along the sight lines vs. target distance $D$. These averages were evaluated as $\left\langle n_{\mathrm{H}}\right\rangle=\sum N_{\mathrm{H}} / \sum D$ and $\left\langle f_{\mathrm{H} 2}\right\rangle=\sum 2 N_{\mathrm{H} 2} / \sum\left[N_{\mathrm{HI}}+\right.$ $\left.2 N_{\mathrm{H} 2}\right]$. Both parallax and photometric distance are shown in the figure, with error bars reflecting formal parallax uncertainties from Gaia-DR2. With many sight lines to bright OB stars ( $D \leq 2 \mathrm{kpc}$ ), the Copernicus survey showed an increase of $f_{\mathrm{H} 2} \geq 0.01$ at $E(B-V) \geq 0.08$ (Savage et al. 1977). The FUSE survey includes more distant sight lines, with column densities up to $\log N_{\mathrm{H}} \approx 21.65$. We found a transition at $f_{\mathrm{H} 2} \geq 0.1$ at $E(B-V) \gtrsim 0.2, N_{\mathrm{H}} \gtrsim 10^{21} \mathrm{~cm}^{-2}$ and $N_{\mathrm{H} 2} \gtrsim 10^{19.5} \mathrm{~cm}^{-2}$. The molecular fractions rise from $f_{H 2} \approx 1 \%$ up to $40-75 \%$ in translucent sight lines with $\log N_{\mathrm{H}} \approx 21.40$ to 21.65 . Using new photometric distances toward 129 stars at $D_{\text {phot }} \leq 5 \mathrm{kpc}$, we find a sightline-averaged hydrogen density $\left\langle n_{\mathrm{H}}\right\rangle=0.50 \mathrm{~cm}^{-3}$ and molecular fraction $\left\langle f_{\mathrm{H} 2}\right\rangle=0.20$. These mean values shift toward higher values (Table 5) in sub-samples with $D_{\text {phot }} \leq 2 \mathrm{kpc}$, owing to bias in distant targets that avoid heavily reddened gas. For stars at $D \leq 2 \mathrm{kpc}$, $\left\langle n_{\mathrm{H}}\right\rangle=0.81 \mathrm{~cm}^{-3}$ and molecular fraction $\left\langle f_{\mathrm{H} 2}\right\rangle=0.27$.

\subsection{Hydrogen Gas vs. Extinction: $N_{\mathrm{H}} / E(B-V)$ Ratio}

The optical extinction along Galactic sight lines is often taken to be proportional to the dust column density, and therefore to the gas column density. This "gas-to-dust ratio" assumes a homogeneous mixture of interstellar hydrogen and grains, which may be a good assumption for most regions of the diffuse ISM. Deviations can be produced by changes in the grain size distribution and other physical properties that arise within dark clouds such as $\rho$ Oph (Bohlin et al. 1978; Green et al. 1992) or in regions where shock waves have sputtered or destroyed some of the grains (Seab \& Shull 1983). The total hydrogen column density, $N_{\mathrm{H}}=N_{\mathrm{HI}}+2 N_{\mathrm{H} 2}$, is often compared to dust content through its ratio to color excess, $N_{\mathrm{H}} / E(B-V)$, derived from UV surveys of $\mathrm{H} \mathrm{I}(\mathrm{Ly} \alpha)$ and $\mathrm{H}_{2}$ toward early-type stars (Bohlin et al. 1978; Savage et al. 1977).

Our FUSE survey of the Milky Way disk should be more robust, with more stellar sight lines, updated O-star photometry and SpTs from the GOS survey, and newly derived values of $E(B-V)$ and target distances (Shull \& Danforth 2019). Figure 6 shows the distribution of $N_{H} / E(B-V)$ vs. $E(B-V)$. For 129 stars at $D \leq 5 \mathrm{kpc}$, we find a mean ratio, $\left\langle N_{\mathrm{H}} / E(B-V)\right\rangle=(6.07 \pm 1.01) \times 10^{21} \mathrm{~cm}^{-2} \mathrm{mag}^{-1}$ with (rms) variations shown as blue wash. A sub-sample of 56 stars at $D \leq 2 \mathrm{kpc}$ (Table 5 ) has $\left\langle N_{\mathrm{H}} / E(B-V)\right\rangle=$ $6.00 \times 10^{21} \mathrm{~cm}^{-2} \mathrm{mag}^{-1}$. Both values are slightly above the values $5.8 \times 10^{21} \mathrm{~cm}^{-2} \mathrm{mag}^{-1}$ in the Copernicus survey of 75 stars (Bohlin et al. 1978) and $5.94 \times 10^{21} \mathrm{~cm}^{-2} \mathrm{mag}^{-1}$ in the FUSE survey of 38 translucent sight lines with $A_{V} \approx 0.5-4.7$ (Rachford et al. 2009).

Recent studies find a larger ratio when $N_{\mathrm{HI}}$ is measured from 21-cm emission and $E(B-V)$ is inferred from all-sky maps (Schlegel et al. 1998; Schlafly \& Finkbeiner 2011) of far-infrared (FIR) dust emission from IRAS and $C O B E / D I R B E$. Liszt $(2014 \mathrm{a}, \mathrm{b})$ found $N_{\mathrm{HI}} / E(B-V)=8.3 \times 10^{21} \mathrm{~cm}^{-2} \mathrm{mag}^{-1}$ in high-latitude sight lines $\left(|b|>20^{\circ}\right)$ with low extinction, $0.015<E(B-V)<0.075$. Lenz et al. (2017) found a similar large value, $8.8 \times 10^{21} \mathrm{~cm}^{-2} \mathrm{mag}^{-1}$. These studies used only $\mathrm{H} \mathrm{I}$, but as the authors comment, corrections for $\mathrm{H}_{2}$ are normally small for $E(B-V)<0.08$.

We investigated whether sight lines with low $E(B-V)$ have different dust-to-gas ratios in the UV surveys. For 25 stars with $0.01 \leq E(B-V) \leq 0.08$ in the Copernicus/IUE survey (Bohlin et al. 1978) the 
mean ratio is $4.0 \times 10^{21} \mathrm{~cm}^{-2} \mathrm{mag}^{-1}$. For the 21 stars in our FUSE survey with $E(B-V) \leq 0.25$, we find a mean ratio $N_{\mathrm{H}} / E(B-V)=5.83 \times 10^{21} \mathrm{~cm}^{-2} \mathrm{mag}^{-1}$. We omitted one outlier (HD 3827) with $\log N_{\mathrm{H}}=20.55$, with an uncertain $E(B-V) \approx 0.02$, and a high ratio $N_{\mathrm{H}} / E(B-V) \approx 17 \times 10^{21} \mathrm{~cm}^{-2} \mathrm{mag}^{-1}$. Located at photometric distance $D_{\text {phot }} \approx 1.88 \mathrm{kpc}$, HD 3827 lies $700-800 \mathrm{pc}$ below the Galactic plane at $b=-23.21^{\circ}$. Its color excess, $E(B-V)=0.02$, was based on magnitudes $B=7.76$ and $V=8.01$ (Deutschman et al. 1976) and intrinsic color $(B-V)_{0}=-0.27$. Jenkins (2019) listed $E(B-V)=0.05$ for this star, based on $B=7.76$ and $V=7.95$, which would reduce the ratio to $7 \times 10^{21} \mathrm{~cm}^{-2} \mathrm{mag}^{-1}$.

The difference between the two techniques (UV absorption and radio/FIR emission) appears to be an effect only seen at high Galactic latitudes (Liszt 2014b; Hensley \& Draine 2021). Elevated ratios from UV data do not appear toward disk stars with low $E(B-V)$. We suggest that the high ratios in 21$\mathrm{cm} /$ FIR measurements result from different distributions of gas and dust above the disk. Dust grains are produced by stars in the disk and grow in the ISM through accretion of refractory elements. Some grains are transported above the disk plane by radiation pressure and supernova-driven outflows. Other grains may settle gravitationally into lower scale-height distributions, separating from the high-latitude H I. Dust at high latitudes may also come into contact with hot coronal gas at $10^{6-7} \mathrm{~K}$ and experience erosion by thermal sputtering and destruction by fast interstellar shock waves (Jones et al. 1996; Slavin et al. 2004). In hot, low-density halo gas, grain lifetimes from sputtering are $t_{\mathrm{sp}} \approx(1 \mathrm{Gyr})\left(10^{-3} \mathrm{~cm}^{-3} / n_{e}\right)$. Thus, the 21-cm and far-IR surveys at $|b|>20^{\circ}$ likely probe systematically lower dust-to-gas ratios.

\subsection{Atomic to Molecular Transition}

As we will describe, the molecular transition from $\mathrm{H}$ I to $\mathrm{H}_{2}$ is consistent with models involving $\mathrm{H}_{2}$ self-shielding and efficient $\mathrm{H}_{2}$ formation by atomic processes on grain surfaces (Hollenbach et al. 1971; Jura 1975a,b; Shull \& Beckwith 1982). In equilibrium, the abundance ratio, $n_{\mathrm{H} 2} / n_{\mathrm{H}}$, can be expressed as a balance between $\mathrm{H}_{2}$ formation and photo-dissociation. For number densities $n_{\mathrm{HI}}, n_{\mathrm{H} 2}$, and total hydrogen $n_{\mathrm{H}}$, molecule formation occurs at a rate per unit volume, $R n_{\mathrm{H}} n_{\mathrm{HI}}$. The total hydrogen density serves as a proxy for dust grains, whose surfaces are catalysts for $\mathrm{H}_{2}$ formation. The coefficient $R$ depends on the dust-to-gas ratio, metallicity, and atom-grain collisional rates, and it likely varies with gas temperature, grain temperature, and surface physics (Hollenbach \& McKee 1979).

Previous studies (Browning et al. 2003; Krumholz et al. 2008, 2009; Sternberg et al. 2014; Bialy \& Sternberg 2016) analyzed the molecular transition with radiative transfer. Here, we present a simple analytic description of the transition (at $f_{\mathrm{H} 2} \approx 0.1$ ) tied directly to parameters that control the $\mathrm{H}_{2}$ equilibrium between formation and destruction and the attenuation of FUV flux by $\mathrm{H}_{2}$ self-shielding and dust opacity. In equilibrium,

$$
n_{\mathrm{HI}} n_{\mathrm{H}} R=\left(f_{\mathrm{diss}} G \beta_{0}\right) n_{\mathrm{H} 2} S_{\mathrm{H} 2} e^{-\tau_{d}},
$$

where $\beta_{0}$ is the average unshielded absorption rate of $\mathrm{H}_{2}$ in the Lyman and Werner bands. For the local ISM, Jura (1974) estimated $\beta_{0}=5 \times 10^{-10} \mathrm{~s}^{-1}$ with $f_{\text {diss }} \approx 0.11$. With updated line-dissociation data and models of self-shielding, Draine \& Bertoldi (1996) found a mean fraction $\left\langle f_{\text {diss }}\right\rangle \approx 0.15$ for all $\mathrm{H}_{2}$ absorptions inside the cloud. The parameter $G$ allows for local elevation of the FUV radiation relative to its average value (Habing 1968), $\tau_{d}$ is the dust optical depth at 930-1130 $\AA$, and the factor $S_{\mathrm{H} 2}$ accounts for $\mathrm{H}_{2}$ selfshielding as the absorption lines become optically thick. For clarity, we define the local molecular fraction $f=2 n_{\mathrm{H} 2} / n_{\mathrm{H}}$, with $n_{\mathrm{HI}}=(1-f) n_{\mathrm{H}}$. This fraction varies with depth into the cloud, whereas the observed 
fraction, $f_{\mathrm{H} 2} \equiv 2 N_{\mathrm{H} 2} / N_{\mathrm{H}}$, depends on the integrated column densities. This leads to an expression,

$$
\frac{f}{(1-f)}=\left(\frac{2 n_{\mathrm{H}} R}{f_{\mathrm{diss}} \beta_{0} G}\right) S_{\mathrm{H} 2}^{-1} e^{\tau_{d}}
$$

Draine \& Bertoldi (1996) provided a reliable approximation, $S_{\mathrm{H} 2}=\left[N_{\mathrm{H} 2} / 10^{14} \mathrm{~cm}^{-2}\right]^{-0.75}$, for $N_{\mathrm{H} 2} \geq$ $10^{14} \mathrm{~cm}^{-2}$. Combining $S_{\mathrm{H} 2}=A N_{\mathrm{H} 2}^{-0.75}\left(A=3.16 \times 10^{10} \mathrm{~cm}^{3 / 2}\right)$ with the approximation, $f \approx 2 n_{\mathrm{H} 2} / n_{\mathrm{H}}$ for $f \leq 0.1$, we can write the local molecular density as

$$
n_{\mathrm{H} 2}=\left(\frac{R n_{\mathrm{H}}^{2}}{f_{\mathrm{diss}} \beta_{0} G A}\right) N_{\mathrm{H} 2}^{0.75} e^{\tau_{d}} .
$$

In a planar slab of constant density $n_{\mathrm{H}}$, with column density $N_{\mathrm{H} 2}$ at a distance $x$ into the absorber, we can write $n_{\mathrm{H} 2}=d N_{\mathrm{H} 2} / d x$. The dust optical depth is $\tau_{d}(x) \approx n_{\mathrm{H}} x / N_{d}$, where $N_{d} \approx 4.5 \times 10^{20} \mathrm{~cm}^{-2}$ at $\lambda \approx 1000-1100 \AA$ (Draine 2011). This leads to a differential equation,

$$
\frac{d N_{\mathrm{H} 2}}{d x}=\left(\frac{R n_{\mathrm{H}}^{2}}{f_{\mathrm{diss}} \beta_{0} G A}\right) N_{\mathrm{H} 2}^{0.75} e^{n_{\mathrm{H}} x / N_{d}},
$$

with the analytic solution

$$
N_{\mathrm{H} 2}=\left(\frac{R N_{d} n_{\mathrm{H}}}{4 f_{\text {diss }} \beta_{0} G A}\right)^{4}\left[e^{\tau_{d}(x)}-1\right]^{4} .
$$

The power-law approximation for $S_{\mathrm{H} 2} \propto N_{\mathrm{H} 2}^{-0.75}$ breaks down at low column densities, since $S_{\mathrm{H} 2}=1$ at $N_{\mathrm{H} 2}<10^{14} \mathrm{~cm}^{-2}$. However, this only occurs in a small surface layer $\left(f_{\mathrm{H} 2}<10^{-5}\right)$. Thus, the above expressions are valid up to to $f_{\mathrm{H} 2}=0.1$ for the $\mathrm{H}_{2}$ absorbers observed with FUSE. The observed atomic-tomolecular transition at $N_{\mathrm{H} 2} \approx 10^{19.5} \mathrm{~cm}^{-2}$ occurs at dust optical depth $\tau_{d}=N_{\mathrm{H}} / N_{d}$ given by

$$
\left[e^{\tau_{d}}-1\right]=(1.755)\left(\frac{N_{\mathrm{H} 2}}{10^{19.5} \mathrm{~cm}^{-2}}\right)^{1 / 4} G n_{30}^{-1}\left(\frac{3 \times 10^{-17} \mathrm{~cm}^{3} \mathrm{~s}^{-1}}{R}\right)\left(\frac{4.5 \times 10^{20} \mathrm{~cm}^{-2}}{N_{d}}\right)
$$

Here, we adopted $f_{\text {diss }}=0.15$ and $A / N_{d}=7.03 \times 10^{-11} \mathrm{~cm}^{1 / 2}$ for metallicities and grain opacities in the local ISM. We scaled the cloud density to $n_{\mathrm{H}}=\left(30 \mathrm{~cm}^{-3}\right) n_{30}$, appropriate for thermal pressures, $P / k \approx 3000 \mathrm{~cm}^{-3} \mathrm{~K}$, inferred from observations of $\mathrm{C}$ I fine-structure populations (Jenkins \& Tripp 2011). Setting the parenthetical terms equal to unity, we find a dust optical depth $\tau_{d}=1.013$. The right-hand side of equation (8) scales as $\left(G / R N_{d} n_{\mathrm{H}}\right)$, which is insensitive to metallicity if the product $\left(R N_{d}\right)$ remains constant. This would be expected if grain abundances decrease at lower metal abundances. In that case, $N_{d}$ would increase and $R$ would decrease. Thus, the transition should be governed by the FUV/density ratio $\left(G / n_{\mathrm{H}}\right)$. In low-metallicity environments such as the LMC/SMC, the transition will occur at similar $\tau_{d} \approx 1$, but at higher total hydrogen column density, $N_{\mathrm{H}}=\tau_{d} N_{d}$.

Previous studies of $\mathrm{H}_{2}$ abundances estimated that $R \approx 3 \times 10^{-17} \mathrm{~cm}^{3} \mathrm{~s}^{-1}$ (Jura 1975a). The observed transition implies a rate coefficient,

$$
R=\left[\frac{4 f_{\mathrm{diss}} \beta_{0} G A}{N_{d} n_{\mathrm{H}}}\right] N_{\mathrm{H} 2}^{1 / 4}\left[e^{\tau_{d}}-1\right]^{-1} \approx\left(3.0 \times 10^{-17} \mathrm{~cm}^{3} \mathrm{~s}^{-1}\right) G n_{30}^{-1}\left[\frac{N_{\mathrm{H} 2}}{10^{19.5}}\right]^{1 / 4},
$$

for $\tau_{d} \approx 1$, as found above. This result suggests that absorbers with densities $n_{\mathrm{H}}>30 \mathrm{~cm}^{-3}$ are associated with elevated radiation fields $(G>1)$ from their proximity to hot stars.

We see that dust can be an important factor, along with $\mathrm{H}_{2}$ self-shielding, in the onset of the molecular transition. Our results show that the transition occurs when $\tau_{d} \approx 1$. Because the dust-to-gas ratio depends 
on abundances of $\mathrm{C}, \mathrm{Si}, \mathrm{O}$, and other refractory heavy elements, changes in metallicity will have offsetting effects on the $\mathrm{H}_{2}$ fractions through the formation coefficient $(R)$ and radiative attenuation $\left(\tau_{d}\right)$. This analysis may explain the observed hydrogen column densities of the molecular transition at $f_{\mathrm{H} 2}=0.1$, which occurs at $\log N_{\mathrm{H}} \approx 21.0$ in the Milky Way, $\log N_{\mathrm{H}} \approx 21.3$ in the LMC, and $\log N_{\mathrm{H}} \approx 22.0$ in the SMC. Sight lines at high Galactic latitude (Gillmon et al. 2006) showed a transition at lower columns ( $\log N_{\mathrm{H}} \approx 20.4$ ), likely because of lower radiation-to-density ratios, and possibly one-sided absorber illumination from the disk stars. The current FUSE survey of the Milky Way disk exhibits no obvious shift with distance, but large changes in metallicity are not expected over the range of target star distances.

More precise determinations of $R$ require knowledge of $n_{\mathrm{H}}$, FUV radiation field, and dust properties, which may depend on metallicity and environment. Interstellar clouds are inhomogeneous, with internal variations in temperature, hydrogen density, and metallicity. Irradiated cloud models have been constructed (Browning et al. 2003; Le Petit et al. 2006; Nehmé et al. 2008; Klimenko \& Balashev 2020) that follow the attenuation of UV radiation into the cloud and the resulting changes in gas temperature $\left(T_{g}\right)$, dust temperature $\left(T_{d}\right)$ and molecular fraction. These models depend sensitively on $n_{\mathrm{H}}, \beta_{0}, G$, and $R\left(T_{g}, T_{d}, Z\right)$. The best measures of the FUV radiation field and cloud density are the high- $J$ excitation ratios, $N(4) / N(2)$ and $N(5) / N(3)$. These issues are discussed further in Section 3.5.

\subsection{Rotational Excitation Temperatures $(J=0,1,2)$}

The excitation temperature $T_{01}$ of the lowest two rotational states, $J=0$ (para- $\mathrm{H}_{2}$ ) and $J=1$ (ortho$\mathrm{H}_{2}$ ) is frequently used as a measure of the kinetic temperature in diffuse clouds. This requires that the gas density and column density be sufficiently high for thermal proton collisions (Gerlich 1990) to couple the ortho and para forms of $\mathrm{H}_{2}$ and set the ratio $N(1) / N(0)$. Recent experiments suggest that ortho-para conversion might occur on silicate grain surfaces (Tsuge et al. 2021). The expectation is that ortho/para production on grains would be in the $3: 1$ spin-statistical ratio. The observed low values of $T_{01} \approx 70-90 \mathrm{~K}$ suggest that this formation channel is subdominant. Theoretical models of $\mathrm{H}_{2}$ formation and destruction in diffuse clouds find that the lowest three rotational states $(J=0,1,2)$ are usually thermalized, with populations depending primarily on gas temperature. We assume that the observed populations obey Boltzmann ratios, with $T_{01}$ and $T_{02}$ determined from the expressions,

$$
\begin{aligned}
T_{01} & =\frac{\Delta E_{01} / k}{\ln \left[\left(g_{1} / g_{0}\right) N(0) / N(1)\right]}, \\
T_{02} & =\frac{\Delta E_{02} / k}{\ln \left[\left(g_{2} / g_{0}\right) N(0) / N(2)\right]} .
\end{aligned}
$$

Here, $g_{1} / g_{0}=9$ and $g_{2} / g_{0}=5$ are ratios of statistical weights of the rotational levels, and $\Delta E_{01} / k=170.48 \mathrm{~K}$ and $\Delta E_{02} / k=509.86 \mathrm{~K}$ come from the rotational energies computed by Komasa et al. (2011).

The initial Copernicus study of $\mathrm{H}_{2}$ in 13 clouds with $N(0) \geq 10^{17} \mathrm{~cm}^{-2}$ found a mean temperature $\left\langle T_{01}\right\rangle=81 \pm 13 \mathrm{~K}$ (Spitzer \& Cochran 1973). A more extensive Copernicus survey (Savage et al. 1977) of 61 stars with $\log N_{\mathrm{H} 2} \geq 18$ found $\left\langle T_{01}\right\rangle=77 \pm 17 \mathrm{~K}$ (rms). In their study of $\mathrm{CO}$ and $\mathrm{H}_{2}$ in diffuse molecular clouds, Sheffer et al. (2008) tabulated total $\mathrm{H}_{2}$ column densities $N_{\mathrm{H} 2}$ along 58 sight lines studied with FUSE. For 24 stars in common with our 139, there was reasonable agreement in $\log N_{\mathrm{H} 2}$, typically within \pm 0.10 . We also compared our $\mathrm{H}_{2}$ column densities to those in the metallicity study (O, Ge, Kr) of Jenkins (2019). For 57 stars in common, we found near agreement (within \pm 0.03 ) for $\log N(0)$ and $\log N(1)$ in 22 and 18 sight lines, respectively. For the other stars, the column densities agreed within \pm 0.10 . We also compared 
the tabulated rotational temperatures in these two studies. Sheffer et al. (2008) quoted a mean rotational temperature, $\left\langle T_{01}\right\rangle=76 \pm 14 \mathrm{~K}$ for 56 sight lines, similar to the Copernicus result of $77 \pm 17 \mathrm{~K}$ (Savage et al. 1977). In our current FUSE survey of 139 stars, we find $\left\langle T_{01}\right\rangle=88 \pm 20 \mathrm{~K}$. Comparing to the 57 common sight lines with Jenkins (2019), we found only a few differences. These are attributable to different values of $N(0)$ and $N(1)$ arising from fitting the damping wings. Sheffer et al. (2008) did not tabulate individual column densities, $N(0)$ and $N(1)$, and we were unable to investigate the source of the differences.

Figure 7 shows the distributions of $T_{01}$ with $E(B-V)$ and $N_{\mathrm{H}}$. Removing the four labeled outliers reduces the mean slightly to $\left\langle T_{01}\right\rangle=87 \mathrm{~K}$. The distributions of $T_{02}$ are shown in Figure 8. For the reduced sample (four outliers removed) of 128 stars with measured $J=2$ column densities, we find $\left\langle T_{02}\right\rangle=77 \pm 18 \mathrm{~K}$. In their analysis of $\mathrm{H}_{2}$ in the LMC and SMC, Tumlinson et al. (2002) found $\left\langle T_{01}\right\rangle=82 \pm 21 \mathrm{~K}$ for 22 sight lines with $N_{\mathrm{H} 2} \geq 10^{16.5} \mathrm{~cm}^{-2}$. Kruczek et al. (2019) explored the contributions of higher rotational lines to the damping wings of $J=1$ lines. Their re-analysis of nine Copernicus sight lines and 13 from FUSE altered $N(0)$ and $N(1)$, resulting in a reduced $T_{01}=68 \pm 13 \mathrm{~K}$ (12\% lower than their previous values). Our FUSE survey extends to larger column densities and greater stellar distances than Copernicus and contains more than twice the number of stars. Given the dispersions in $T_{01}$ and $T_{02}$ distributions, their agreement suggests that the diffuse ISM has similar heating and cooling rates over a wide range of cloud densities, metallicities, and FUV radiation fields.

Even for the best determinations of $\log N(0)$ and $\log N(1)$, rotational temperatures $T_{01}$ have errors of $\sigma_{T_{01}} \approx 4 \mathrm{~K}$. Uncertainties on $T_{02}$ can be higher, when $\log N(2)$ is poorly determined. From errors on $\log N_{0}$, $\log N_{1}$, and $\log N_{2}$, and neglecting covariance, the propagated errors on $T_{01}$ and $T_{02}$ derived from equations (10) and (11) are:

$$
\begin{aligned}
\frac{\sigma_{T_{01}}}{T_{01}} & =2.303\left[\frac{T_{01}}{170.48 \mathrm{~K}}\right]\left[\sigma_{\log N_{0}}^{2}+\sigma_{\log N_{1}}^{2}\right]^{1 / 2} \\
\frac{\sigma_{T_{02}}}{T_{02}} & =2.303\left[\frac{T_{02}}{509.86 \mathrm{~K}}\right]\left[\sigma_{\log N_{0}}^{2}+\sigma_{\log N_{2}}^{2}\right]^{1 / 2} .
\end{aligned}
$$

For equal errors on $J=0$ and $J=1$ column densities, $\sigma_{\log N_{1}}=\sigma_{\log N_{0}}$, this expression simplifies to $\sigma_{T_{01}} \approx\left[T_{01}^{2} / 52.34\right] \sigma_{\log N_{0}}$. For the best fits to the damping wings $\left(\sigma_{\log N_{0}}=0.03\right)$ the temperature uncertainty is $\sigma_{T_{01}}=4.3 \mathrm{~K}$ at $T_{01} \approx 87 \mathrm{~K}$. In poorer quality data, with $\sigma_{\log N_{0}} \approx \sigma_{\log N_{1}}=0.07$, the uncertainty is higher, $\sigma_{T_{01}} \approx 10 \mathrm{~K}$. Errors on $J=2$ column densities are usually much larger than those for $J=0$. Thus, $\sigma_{T_{02}} \approx\left[T_{02}^{2} / 221.39\right] \sigma_{\log N_{2}}$. At $T_{02} \approx 77 \mathrm{~K}$, the uncertainty $\sigma_{T_{02}} \approx 5-11 \mathrm{~K}$ for $\sigma_{\log N_{2}}=0.20-0.40$.

\subsection{Rotational Excitation Temperatures $(J \geq 3)$}

The higher rotational levels $(J \geq 3)$ of $\mathrm{H}_{2}$ are generally believed to be populated by the fluorescent cascade following FUV radiative pumping in the Lyman and Werner bands (Black \& Dalgarno 1976; Spitzer \& Zweibel 1974; Jura 1974). The FUV radiation field includes the ambient Galactic radiation field (Jura 1974; Habing 1968; Draine 2011) augmented by local flux from O stars. Measurements by Copernicus (Spitzer et al. 1974; Morton 1975) found that levels $J \geq 3$ were populated in excess of predictions from $T_{01}$. The column densities in $J=3,4,5$ were fitted to excitation temperatures $T_{\text {exc }} \approx 200-500 \mathrm{~K}$, and sometimes as high as $1100 \mathrm{~K}$ near luminous early O-type stars such as Zeta Puppis (Morton \& Dinerstein 1976). Subsequent studies of $\mathrm{H}_{2}$ excitation using FUSE data (Browning et al. 2003; Sheffer et al. 2008; Nehmé et al. 2008; Jensen et al. 2010) also found $T_{\mathrm{exc}} \approx 200-500 \mathrm{~K}$ for $J \geq 3$. The mean rotational excitation temperature, fitted to $J \geq 3$ and averaged over our sample, is $T_{\text {exc }}=326 \pm 125 \mathrm{~K}$ with a typical 
range from 150-650 K. These temperatures are similar to those seen toward selected Copernicus targets, and they likely reflect fluorescent pumping of high- $J$ states. Some observations suggest that $J \geq 2$ levels may be influenced by collisional excitation (Gry et al. 2002; Nehmé et al. 2008; Ingalls et al. 2011) in a component of warm gas $(T>500 \mathrm{~K})$ heated by turbulent dissipation (Moseley et al. 2020). Observationally, $\mathrm{CH}^{+}$is correlated with rotationally-excited $\mathrm{H}_{2}$ (Jensen et al. 2010), leading to suggested production schemes for $\mathrm{CH}^{+}$involving hot $\mathrm{H}_{2}$ (Falgarone \& Puget 1995; Myers et al. 2015). Sheffer et al. (2008) tabulated several excitation temperatures to higher rotational states $\left(T_{03}\right.$ and $\left.T_{04}\right)$ for 56 FUSE sight lines. We chose not to tabulate these parameters, since our modeling experience (Browning et al. 2003) found them less useful than $T_{24}$ and $T_{35}$. Many of their values of $T_{04}$ seemed implausibly high (200-300 K) and inconsistent with our observed ratios of $N(4) / N(0)$. Because they did not tabulate individual values of $N(J)$, we could not investigate further.

In our survey, we choose to focus on individual pairs of upper $(u)$ and lower $(l)$ rotational states $\left(J_{l}, J_{u}\right)$, in particular $(0,2),(2,4)$, and $(3,5)$. These parameters capture the fact that $\mathrm{H}_{2}$ exists over a range of cloud temperatures, with radiative pumping changing throughout the cloud because of $\mathrm{H}_{2}$ self-shielding and attenuation by FUV extinction. In addition, the rate of ortho-para conversion may change, depending on cloud density. This will affect radiative pumping from $J=0, J=1$, and sometimes $J=2$, creating departures of rotational populations of $J \geq 3$ from a single excitation temperature. For these reasons, we tabulate pairwise excitation temperatures, $T_{02}, T_{24}$, and $T_{35}$, each remaining within para (even- $J$ ) and ortho (odd- $J$ ) forms of $\mathrm{H}_{2}$. The temperatures $T_{04}$ and $T_{15}$ are not as useful diagnostics. Detailed models of the excitation processes and radiative transfer may help to distinguish the relative contributions of FUV pumping and collisional excitation and to estimate the FUV radiation field and gas density.

Based on column densities $N(J)$ in the FUSE survey, Table 4 lists four excitation temperatures, $T_{01}$, $T_{02}, T_{24}$, and $T_{35}$, between upper and lower rotational states defined by

$$
T_{l u}=\frac{\left(E_{u}-E_{l}\right) / k}{\ln \left[\left(g_{u} / g_{l}\right)\left(N_{l} / N_{u}\right)\right]},
$$

corresponding to Boltzmann population ratios,

$$
\begin{aligned}
& N(1) / N(0)=(9 / 1) \exp \left[-170.48 \mathrm{~K} / T_{01}\right] \\
& N(2) / N(0)=(5 / 1) \exp \left[-509.86 \mathrm{~K} / T_{02}\right] \\
& N(4) / N(2)=(9 / 5) \exp \left[-1171.78 \mathrm{~K} / T_{24}\right] \\
& N(5) / N(3)=(11 / 7) \exp \left[-1488.66 \mathrm{~K} / T_{35}\right] .
\end{aligned}
$$

These excitation temperatures were derived from the relativistic quantum calculations of Komasa et al. (2011), using the $J$-level dissociation energies in their Table 1 . From the observed populations of higher- $J$ states, we find mean excitation temperatures, $\left\langle T_{24}\right\rangle=237 \pm 91 \mathrm{~K}$ and $\left\langle T_{35}\right\rangle=304 \pm 108 \mathrm{~K}$. Even with a wide range of these temperatures, they generally exhibit a correlation between the two parameters.

Figure 9 shows the rotational distributions in four of the six sight lines with detections up to $J=7$. These include Star \#48 (HD 93250) with $\log N(7)=15.54 \pm 0.15$; Star \#88 (HD 163892) with $\log N(7)=$ $14.14 \pm 0.06$; Star \#116 (HD 199579) with $\log N(7)=14.29 \pm 0.11$; and Star \#137 (HD 303308) with $\log N(7)=15.43 \pm 0.15$. The absorbers along these sight lines have different excitation temperatures. Two exhibit higher excitation temperatures for $J=4$ and $J=5$, likely produced by elevated FUV radiation fields from absorber proximity to hot stars.

Figure 10 displays distributions of the four excitation temperatures with stellar target distance. We see no obvious trend with increasing distance, although stars beyond $4-5 \mathrm{kpc}$ are unlikely to be an unbiased 
sample. The similar values of of $T_{01}$ and $T_{02}$ suggest that the $J=0,1,2$ levels are thermalized to the gas kinetic temperature. Both $T_{24}$ and $T_{35}$ show several outliers, well above their distribution means of $237 \mathrm{~K}$ and $304 \mathrm{~K}$, respectively. For this 10-15\% population, higher rotational excitation is expected from exposure to local FUV radiation above the background.

Figure 11 shows the relation of $T_{24}$ and $T_{35}$, color-coded by the SpT of the target star. With the exception of a few labeled outliers, the para- $\mathrm{H}_{2}$ levels $(J=2$ and 4$)$ and ortho- $\mathrm{H}_{2}$ levels $(J=3$ and 5$)$ have correlated excitation temperatures above $300 \mathrm{~K}$. Most sight lines have $T_{35}>T_{24}$, with data points above the dashed line of unit slope. This difference could arise from strong pumping of ortho- $\mathrm{H}_{2}$ populations out of $J=1$. Alternatively, thermalization of the $J=2$ population to the gas kinetic temperature may alter the pumping out of $J=2$. The expectation that high- $J$ populations would be greater toward hotter O-type stars is not consistently reflected in this plot. The labeled sight lines with $T_{35}>500 \mathrm{~K}$ include two early O-type stars (\#4, \#48), two later O-type stars (\#32, \#122), and two B0/B0.5 stars (\#2, \#59). A number of hot $(\mathrm{O} 2-\mathrm{O} 4)$ stars exhibit high excitation temperatures $\left(T_{35}>450 \mathrm{~K}\right)$, while others have $T_{35}<400 \mathrm{~K}$. This mixed distribution suggests that radiative pumping of $J \geq 3$ levels is sometimes enhanced by proximity of the molecular absorbers to nearby hot stars. Deriving the pertinent model parameters of FUV absorption rate and absorber density will require modeling of the ratios $N(4) / N(2)$ and $N(5) / N(3)$, as performed by Browning et al. (2003) and Klimenko \& Balashev (2020).

\section{Discussion and Summary}

This FUSE survey of interstellar $\mathrm{H}_{2}$ in the Milky Way disk complements the pioneering survey by Copernicus, with several important extensions. Because FUSE was a more sensitive spectrograph, the survey includes more OB-star targets at greater distances and larger $\mathrm{H}_{2}$ column densities. Many FUSE sight lines exhibit molecular fractions above the value, $f_{\mathrm{H} 2} \geq 0.01$ at $\log N_{\mathrm{H}} \geq 20.7$, noted in the Copernicus survey (Savage et al. 1977). We also measured $\mathrm{H}_{2}$ populations in higher rotational states $(J \geq 2)$ as well as $J=0$ and $J=1$. The FUSE sample includes the 139 OB-star targets with updated distances and $E(B-V)$ from Shull \& Danforth (2019) derived from updated spectral types, digital photometry, and optical-NIR dust extinction in the Galactic O-star Spectroscopic Survey (Maíz Apellániz et al. 2004; Maíz-Apellániz \& Barbá 2018). Our measurements of $\mathrm{H}_{2}$ populations in the lowest three rotational states $(J=0,1,2)$ found similar excitation temperatures, $T_{01} \approx T_{02} \approx 70-90 \mathrm{~K}$, suggesting thermal coupling to the gas kinetic temperature. Populations of higher rotational states $(J \geq 3)$ could be use to distinguish between radiative pumping and collisional excitation. The pairwise excitation temperatures, $T_{24}$ and $T_{35}$, are correlated at the high end of the distribution, with $T_{35}>T_{24}$. After deriving total hydrogen column densities, $N_{\mathrm{H}}$, from those of $\mathrm{H}_{2}$ and $\mathrm{H}$ I, we compared them to updated values of selective extinction, $E(B-V)$, to find a mean gas-to-dust ratio in the Galactic disk, $\left\langle N_{\mathrm{H}} / E(B-V)\right\rangle=(6.07 \pm 1.01) \times 10^{21} \mathrm{~cm}^{-2} \mathrm{mag}^{-1}$.

The FUSE survey finds that a typical atomic-to-molecular transition in the ISM of the Galactic disk occurs at molecular fraction $f_{\mathrm{H} 2} \approx 0.1$ and molecular column density $N_{\mathrm{H} 2} \approx 10^{19.5} \mathrm{~cm}^{-2}$. This transition can be understood with a simple analytic model describing its dependence on $\mathrm{H}_{2}$ formation on dust-grain surfaces and photo-dissociation by FUV radiation, including self-shielding and dust attenuation. This formulation shows that the transition depends on the ratio of FUV flux to gas density, analogous to the photoionization parameter used in the analysis of nebular lines. The transition occurs at dust optical depth $\tau_{d} \approx 1$ and total hydrogen column density $N_{\mathrm{H}}=\tau_{d} N_{d}$, where the dust-opacity parameter $N_{d} \approx 4.5 \times 10^{20} \mathrm{~cm}^{-2}$ at solar metallicity $\left(Z \approx Z_{\odot}\right)$. The $\mathrm{H}$ I-to- $\mathrm{H}_{2}$ conversion is mediated by both dust opacity and $\mathrm{H}_{2}$ self-shielding. The optical depth $\tau_{d} \approx 1$ is likely insensitive to metallicity and grain/gas ratio, because of offsetting effects 
of grain surface area on the $\mathrm{H}_{2}$ formation rate coefficient $(R)$ and dust opacity $\left(N_{d}\right)$.

With a mean fractional abundance $\left\langle f_{\mathrm{H} 2}\right\rangle \approx 0.2$ in diffuse interstellar clouds, radiative cooling by $28.22 \mu \mathrm{m}$ emission from the $J=2$ level of $\mathrm{H}_{2}(510 \mathrm{~K}$ excitation) augments the dominant cooling from the $157.74 \mu \mathrm{m}[\mathrm{C} \mathrm{II}]$ fine-structure line $\left(91.21 \mathrm{~K}\right.$ excitation). At low densities, the cooling rate from $\mathrm{H}^{\circ}-\mathrm{H}_{2}$ collisions is $\mathcal{L}_{\mathrm{H} 2}=\left(3.1 \times 10^{-28} \mathrm{erg} \mathrm{cm}^{3} \mathrm{~s}^{-1}\right) n_{\mathrm{HI}} n_{\mathrm{H} 2}$ at $T=100 \mathrm{~K}$ (Forrey et al. 1997), while that from [C II] is $\mathcal{L}_{\mathrm{CII}} \approx\left(3.0 \times 10^{-28} \mathrm{erg} \mathrm{cm}^{3} \mathrm{~s}^{-1}\right) n_{\mathrm{H}}^{2}$. We adopted an electron-impact collision strength $\Omega_{12}=1.56$, a solar carbon abundance $n_{C} / n_{\mathrm{H}}=2.69 \times 10^{-4}$, and electron density $n_{e} \approx 3.3 \times 10^{-4} n_{\mathrm{H}}$ donated by trace metal ions. Thus, $\mathcal{L}_{\mathrm{H} 2} / \mathcal{L}_{\mathrm{CII}} \approx 0.5 f_{\mathrm{H} 2}\left(1-f_{\mathrm{H} 2}\right)$ at $100 \mathrm{~K}$. In higher density clouds, the $\mathrm{H}_{2} 28.22 \mu \mathrm{m}$ emission will be reduced by collisional de-excitation. The $\mathrm{H}_{2}$ cooling rises at $T>100 \mathrm{~K}$, but drops off exponentially in lower temperature clouds at higher $N_{\mathrm{H}}$, owing to the $510 \mathrm{~K}$ excitation temperature of the $J=2$ level.

The following summarizes the results of the FUSE survey of interstellar $\mathrm{H}_{2}$ abundances and inferred physical parameters in the Milky Way disk:

1. The FUSE survey measured column densities of $\mathrm{H}_{2}$ in the Galactic disk toward 139 OB stars with recently updated SpT, photometry, and distances (Shull \& Danforth 2019). The survey extends the Copernicus $\mathrm{H}_{2}$ survey (Savage et al. 1977) up to total hydrogen column densities $N_{\mathrm{H}} \approx 5 \times 10^{21} \mathrm{~cm}^{-2}$ and complements FUSE surveys of $\mathrm{H}_{2}$ in the Magellanic Clouds (Tumlinson et al. 2002), translucent clouds (Rachford et al. 2002, 2009), and gas at high Galactic latitude (Gillmon et al. 2006; Wakker 2006).

2. For each sight line, we report column densities $N_{\mathrm{H} 2}, N_{\mathrm{HI}}, N(J), N_{\mathrm{H}}=N_{\mathrm{HI}}+2 N_{\mathrm{H} 2}$, and $f_{\mathrm{H} 2}=$ $2 N_{\mathrm{H} 2} / N_{\mathrm{H}}$, with mean values listed in Table 5 . The mean gas-to-dust ratio, $\left\langle N_{\mathrm{H}} / E(B-V)\right\rangle=(6.07 \pm$ $1.01) \times 10^{21} \mathrm{~cm}^{-2} \mathrm{mag}^{-1}$, is slightly above the value of $5.8 \times 10^{21} \mathrm{~cm}^{-2} \mathrm{mag}^{-1}$ in the Copernicus survey (Bohlin et al. 1978). The larger ratios seen in 21-cm/far-IR surveys at high Galactic latitudes (Liszt 2014a,b) suggest different distributions of gas and dust above the disk, produced by grain sedimentation to the disk plane or dust destruction when transported above the disk.

3. Using an analytic model of $\mathrm{H}_{2}$ formation-destruction equilibrium with dust opacity and $\mathrm{H}_{2}$ selfshielding, we derive an expression for the atomic-to-molecular transition, which occurs at optical depth $\tau_{d} \approx 1$, molecular fraction $f_{\mathrm{H} 2} \approx 0.1, N_{\mathrm{H} 2} \approx 10^{19.5} \mathrm{~cm}^{-2}$, and $N_{\mathrm{H}} \approx 10^{21} \mathrm{~cm}^{-2}$. An $\mathrm{H}_{2}$ formation rate coefficient $R \approx 3 \times 10^{-17} \mathrm{~cm}^{3} \mathrm{~s}^{-1}$ is consistent with the observed transition, with occasional elevated FUV radiation fields in 10-15\% of the sight lines. These parameters can be constrained with models of $\mathrm{H}_{2}$ rotational populations, supplemented by C I fine-structure abundances (Jenkins \& Tripp 2011; Klimenko \& Balashev 2020).

4. The lowest three rotational states $(J=0,1,2)$ appear to be thermally coupled by collisions to the gas kinetic temperature. The survey mean excitation temperatures are $\left\langle T_{01}\right\rangle=88 \pm 20 \mathrm{~K}$ and $\left\langle T_{02}\right\rangle=$ $77 \pm 18 \mathrm{~K}$. For sight lines with $E(B-V)>0.5$ and $\log N_{\mathrm{H}}>20.7$, these temperatures decrease to $50-70 \mathrm{~K}$.

5. Populations of higher- $J$ states are produced primarily by radiative pumping from FUV radiation. From column-density ratios of rotational levels $N(4) / N(2)$ and $N(5) / N(3)$, we find mean excitation temperatures, $\left\langle T_{24}\right\rangle=237 \pm 91 \mathrm{~K}$ and $\left\langle T_{35}\right\rangle=304 \pm 108 \mathrm{~K}$ (rms). In most cases, these two temperatures are correlated, with $T_{35}>T_{24}$, but we find no consistent connection with SpT (from O3 to B1). Elevated radiative pumping is likely produced by close proximity to hot stars, possibly with some collisional excitation from heating by turbulent dissipation. 
Acknowledgements. This work was supported by the FUSE mission, with financial support from NASA Contract NAS5-32985 to Johns Hopkins University and a sub-contract to the University of Colorado at Boulder. We thank Jason Tumlinson for developing software for FUSE studies of $\mathrm{H}_{2}$ and former CU undergraduates Teresa Ross and Kristen Gillmon for their assistance with data analysis during early stages of this project. We have benefitted from discussions on interstellar gas, molecules, and dust with Sergei Balashev, John Black, Bruce Draine, Kevin France, Ed Jenkins, Slava Klimenko, Harvey Liszt, Chris McKee, Blair Savage, and Don York.

\section{REFERENCES}

Balashev, S. A., Klimenko, V. V., Noterdaeme, P., et al. 2019, MNRAS, 490, 2668

Bialy, S., \& Sternberg, A. 2016, ApJ, 822, 83

Black, J. H., \& Dalgarno, A. 1976, ApJ, 203, 132

Bohlin, R. C., Savage, B. D., \& Drake, J. F. 1978, ApJ, 224, 132

Bowen, D. V., Jenkins, E. B., Tripp, T. M., et al. 2008, ApJS, 176, 59

Browning, M. K., Tumlinson, J., \& Shull J. M. 2003, ApJ, 582, 810

Burgh, E. B., France, K., \& McCandliss, S. R. 2007, ApJ, 658, 446

Dalgarno, A., Black, J. H., \& Weisheit, J. C. 1973, Astrophys. Letters, 14, 77

Deutschman, W. A, Davis, R. T., \& Schild, R. E. 1976, ApJS, 30, 97

Diplas, A., \& Savage, B. D. 1994, ApJS, 93, 211

Draine, B. T. 2011, Physical Processes in the Interstellar and Intergalactic Medium (Princeton: Princeton Univ. Press)

Draine, B. T., \& Bertoldi, F. 1996, ApJ, 468, 269

Falgarone, E., \& Puget, J.-L. 1995, A\&A, 293, 840

Fitzpatrick, E. L., \& Massa, D. 1990, ApJS, 72, 163

Forrey, R. C., Balakrishnan, N., Dalgarno, A., \& Lepp, S. 1997, ApJ, 489, 1000

Gerlich, D. J. 1990, J. Chem. Phys., 92, 2377

Gillmon, K., \& Shull, J. M. 2006, ApJ, 636, 908

Gillmon, K., Shull, J. M., Tumlinson, J., \& Danforth, C. 2006, ApJ, 636, 891

Green, J. C., Snow, T. P., Cook, T. A., Cash, W. C., \& Poplawski, O. 1992, ApJ, 395, 289

Gry, C., Boulanger, F., Nehmé, C., et al. 2002, A\&A, 391, 675

Habing, H. J. 1968, B.A.N., 19, 421

Hanson, M. M., Snow, T. P., \& Black, J. H. 1996, ApJ, 392, 571

Hébrard, G., Tripp, T. M., Chayer, P., et al. 2005, ApJ, 635, 1136

Hensley, B. S., \& Draine, B. T. 2021, ApJ, 906, 73

Hollenbach, D. J., \& McKee, C. F. 1979, ApJS, 44, 555

Hollenbach, D. J., Werner, M. W., \& Salpeter, E. E. 1971, ApJ, 163, 165

Hoopes, C. G., Sembach, K. R., Hébrard, G., Moos, H. W., \& Knauth, D. C. 2003, ApJ, 586, 1094

Ingalls, J. G., Bania, T.M., Boulanger, F., et al. 2011, ApJ, 743, 174

Jensen, A. G., Snow, T. P., Sonneborn, G., \& Rachford, B. L. 2010, ApJ, 711, 1236 
Jenkins, E. B. 2019, ApJ, 872, 55

Jenkins, E. B., \& Peimbert, A. 1997, ApJ, 477, 265

Jenkins, E. B., \& Tripp, T. M. 2011, ApJ, 734, 65

Jones, A. P., Tielens, A. G. G. M., \& Hollenbach, D. J. 1996, ApJ, 469, 740

Jorgenson, R. A., Murphy, M. T., Thompson, R., \& Carswell, R. F. 2014, MNRAS, 443, 2783

Jura, M. 1974, ApJ, 191, 375

Jura, M. 1975a, ApJ, 197, 575

Jura, M. 1975b, ApJ, 197, 581

Klimenko, V. V., \& Balashev, S. A. 2020, MNRAS, 498, 1531

Komasa, J., Piszczatowski, K., Lach, G., et al., 2011, J. Chem. Theory Comput., 7, 3105

Kruczek, N., France, K., Hoadley, K., Fleming, B., \& Nell, N. 2019, ApJ, 878, 77

Krumholz, M. R., McKee, C. F., \& Tumlinson, J. 2008, ApJ, 689, 865

Krumholz, M. R., McKee, C. F., \& Tumlinson, J. 2009, ApJ, 693, 216

Lacour, S., Ziskin, V., Hébrard, G., et al., 2005, ApJ, 627, 251

Le Petit, F., Nehmé, C., Le Bourlot, J., \& Roueff, E. 2006, ApJS, 164, 506

Lenz, D., Hensley, B. S., \& Doré, O. 2017, ApJ, 846, 38

Liszt, H. 2014a, ApJ, 780, 10

Liszt, H. 2014b, ApJ, 783, 17

Maíz Apellániz, J., \& Barbá, R. H. 2018, A\&A, 613, A9

Maíz Apellániz, J., Walborn, N. R., Galué, H. A., \& Wei, L. H. 2004, ApJS, 151, 103

Massa, D., Fullerton, A. J., Sonneborn, G., \& Hutchings, J. B. 2003, ApJ, 586, 996

McCandliss, S. R. 2003, PASP, 115, 651

Moos, H. W., Cash, W. C., Cowie, L. L., et al. 2000, ApJ, 538, L1

Moos, H. W., Sembach, K. R., Vidal-Madjar, A., et al. 2002, ApJS, 140, 3

Morton, D. C. 1975, ApJS, 197, 85

Morton, D. C., \& Dinerstein, H. L. 1976, ApJ, 204, 1

Moseley, E. R., Draine, B. T., Tomida, K., \& Stone, J. M. 2021, MNRAS, 500, 3290

Myers, A. F., McKee, C. F., \& Li, P. S. 2015, MNRAS, 453, 2747

Nehmé, C., Le Bourlot, J., Boulanger, F., et al., 2008, A\&A, 483, 485

Noterdaeme, P., Ledoux C., Petitjean, P., \& Srianand, R. 2008, A\&A, 481, 327

Rachford, B. L., Snow, T. P., Tumlinson, J., et al. 2002, ApJ, 577, 221

Rachford, B. L., Snow, T. P., Destree, J. D., et al. 2009, ApJS, 180, 125

Sahnow, D. J., Moos, H. W., Ake, T. B., et al. 2000, ApJ, 538, L7

Savage, B. D., Bohlin, R. C., Drake, J. F., \& Budich, W. 1977, ApJ, 216, 291

Schlafly, E. F., \& Finkbeiner, D. P. 2011, ApJ, 77, 103

Schlegel, D. J., Finkbeiner, D. P., \& Davis, M. 1998, ApJ, 500, 525

Seab, C. G., \& Shull, J. M. 1983, ApJ, 275, 652

Sheffer, Y., Rogers, M., Federman, S. R., et al. 2008, ApJ, 687, 1075 
Shull, J. M., \& Beckwith, S. 1982, ARA\&A, 20, 163

Shull, J. M., \& Danforth, C. W. 2019, ApJ, 882, 180

Shull, J. M., Tumlinson, J., Jenkins, E. B., et al. 2000, ApJ, 538, L73

Shull, J. M., \& Van Steenberg, M. E. 1985, ApJ, 294, 599

Slavin, J. D., Dwek, E., \& Jones, A .P. 2004, ApJ, 803, 7

Snow, T. P., Black, J. H., van Dishoeck, E. F., et al. 1992, ApJ, 465, 245

Snow, T. P., Rachford, B. L., Tumlinson, J., et al. 2000, ApJ, 538, L65

Sota, A., Maíz Apellániz, J., Morrell, N. I., et al., 2014, ApJS, 211, 10

Sota, A., Maíz Apellániz, J., Walborn, N. R., et al., 2011, ApJS, 193, 24

Spitzer, L., \& Cochran, W. D. 1973, ApJ, 186, L23

Spitzer, L., Cochran, W. D., \& Hirshfeld, A. 1974, ApJS, 28, 373

Spitzer, L., \& Zweibel, E. G. 1974, ApJ, 191, L127

Sternberg, A., Le Petit, F., Roueff, E., \& Le Bourlot, J. 2014, ApJ, 790, 10

Tsuge, M., Namiyoshi, T., Furuya, K., et al. 2021, ApJ, in press (arXiv:2101.02357)

Tumlinson, J., Shull, J. M., Rachford, B. L., et al. 2002, ApJ, 566, 857

Wakker, B. P. 2006, ApJS, 163, 282 

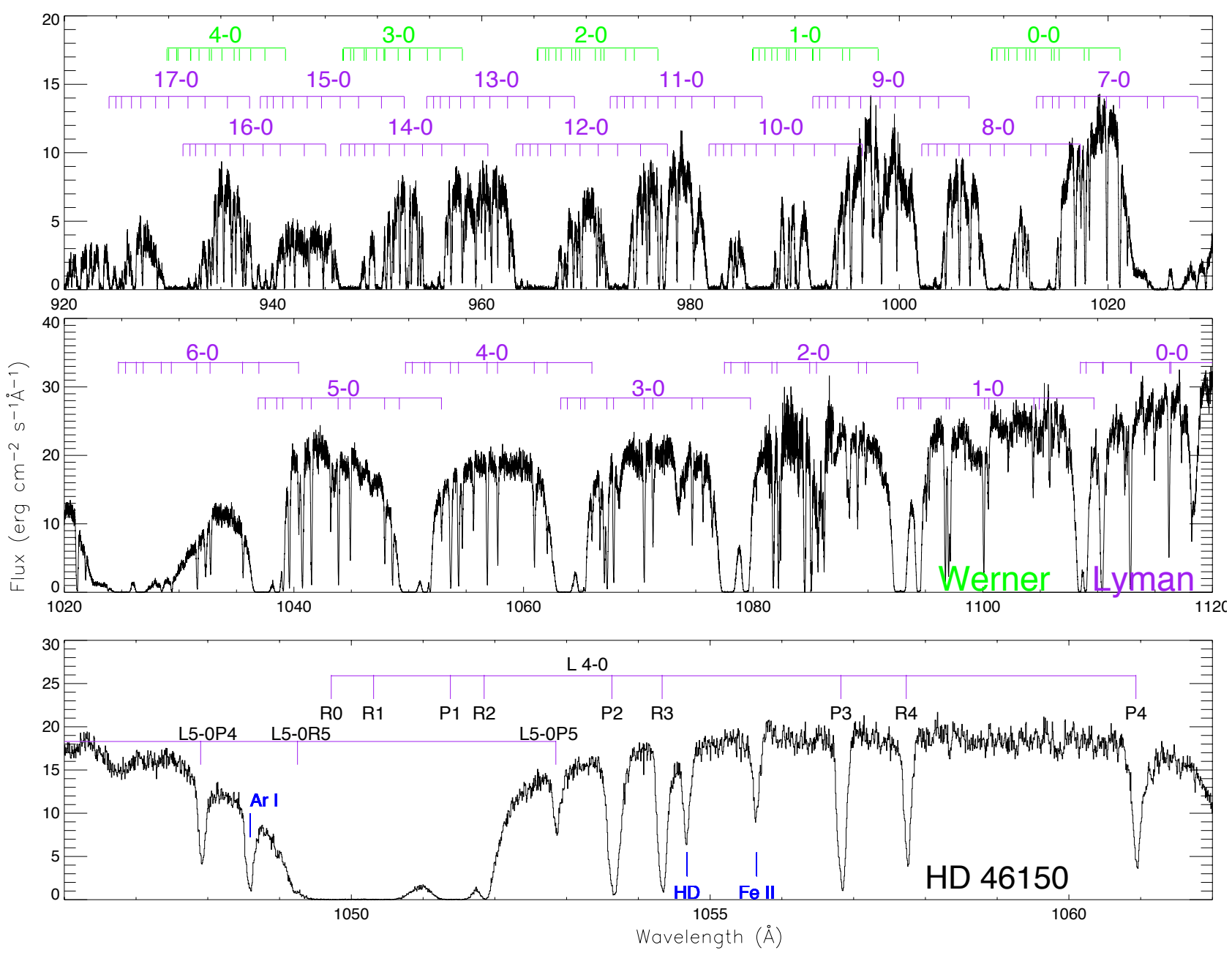

Fig. 1. - FUSE spectrum of the sight line to HD 46150, an O5 Vf star at $1.5 \mathrm{kpc}$ distance and $E(B-V)=$ 0.45 , located inside the Rosette Nebula. Locations of the $\mathrm{H}_{2}$ Lyman and Werner bands are shown as purple and green templates in the top two panels. Each template shows a different vibrational band $\left(v_{u}-v_{\ell}\right)$ with a comb of absorption lines from rotational levels $J$ in the ground vibrational state $\left(v_{\ell}=0\right)$ to the upper vibrational state $\left(v_{u}\right)$ in the excited electronic states, $B^{1} \Sigma_{u}^{+}$(Lyman bands) and $C^{1} \Pi_{u}$ (Werner bands). For each band, the template shows locations of R-branch and P-branch absorption lines from $J=0,1,2, \ldots$. The bottom panel shows a close-up of the (4-0) Lyman band, with nearby lines of Ar I (1048.220 $)$, Fe II (1055.262 $\AA$ ), HD (4-0) $\mathrm{R}(0)$ at $1054.294 \AA$, and $\mathrm{P}(4), \mathrm{R}(5), \mathrm{P}(5)$ lines from $J=4$ and $J=5$ levels of the (5-0) Lyman band. The $\mathrm{R}(0), \mathrm{R}(1), \mathrm{P}(1)$ lines have damping wings and are blended. In some bands, the $\mathrm{P}(1)$ and $\mathrm{R}(2)$ lines are separable. The P-branch and R-branch lines from higher- $J$ states are sufficiently shifted to be measured. We find $\log N_{\mathrm{H} 2}=20.64 \pm 0.04$, with $\log N(0)=20.23 \pm 0.05, \log N(1)=20.42 \pm 0.05$, and rotational temperatures $T_{01}=97 \pm 9 \mathrm{~K}$ and $T_{02}=55 \pm 4 \mathrm{~K}$. 


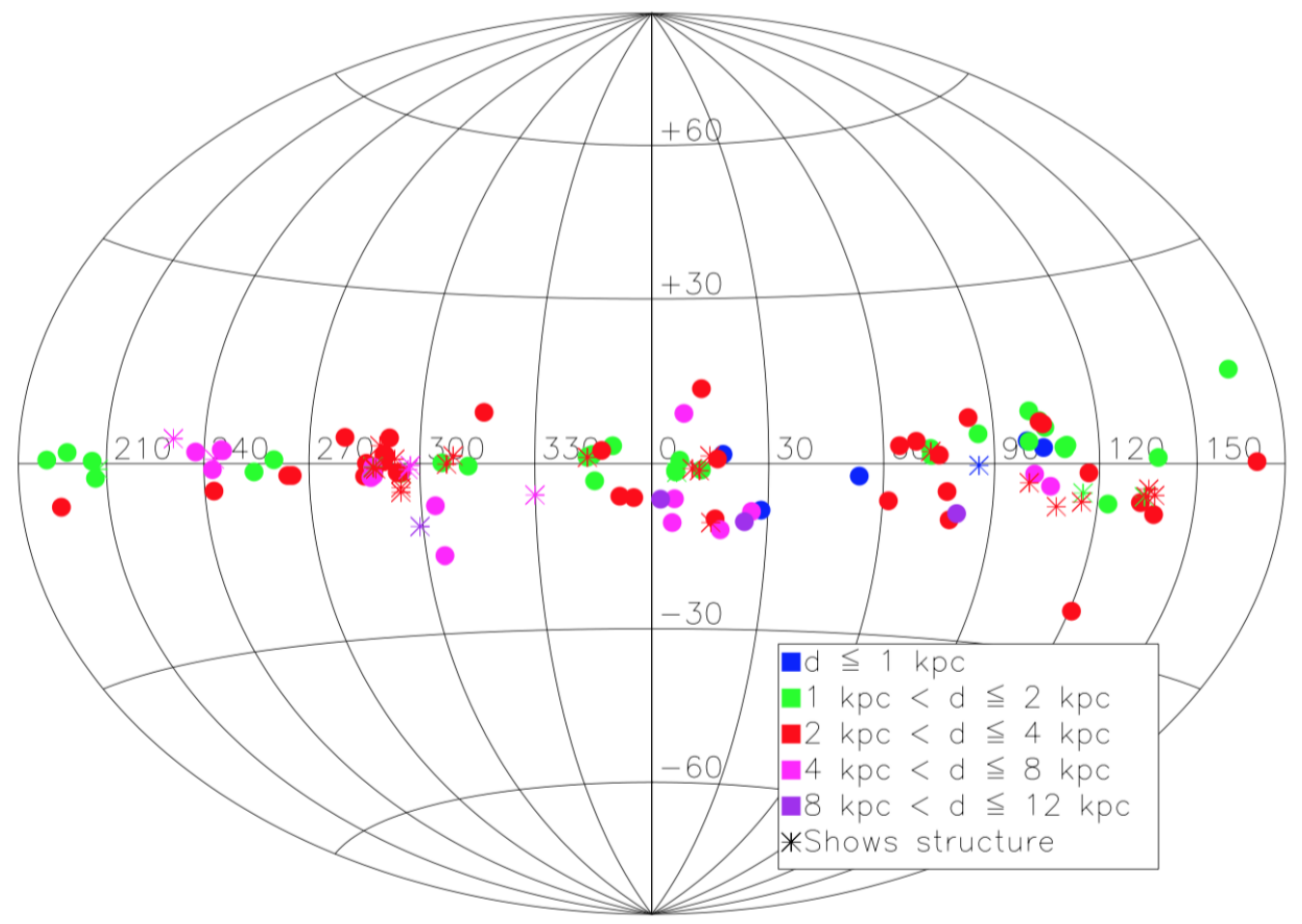

Fig. 2.- Aitoff projection plot of 139 target locations in Galactic coordinates $(\ell$ and $b)$ with distances coded by color. Several sight lines with multiple velocity components that are separably measurable $(\Delta v \geq$ $20 \mathrm{~km} \mathrm{~s}^{-1}$ ) are shown as asterisks. 


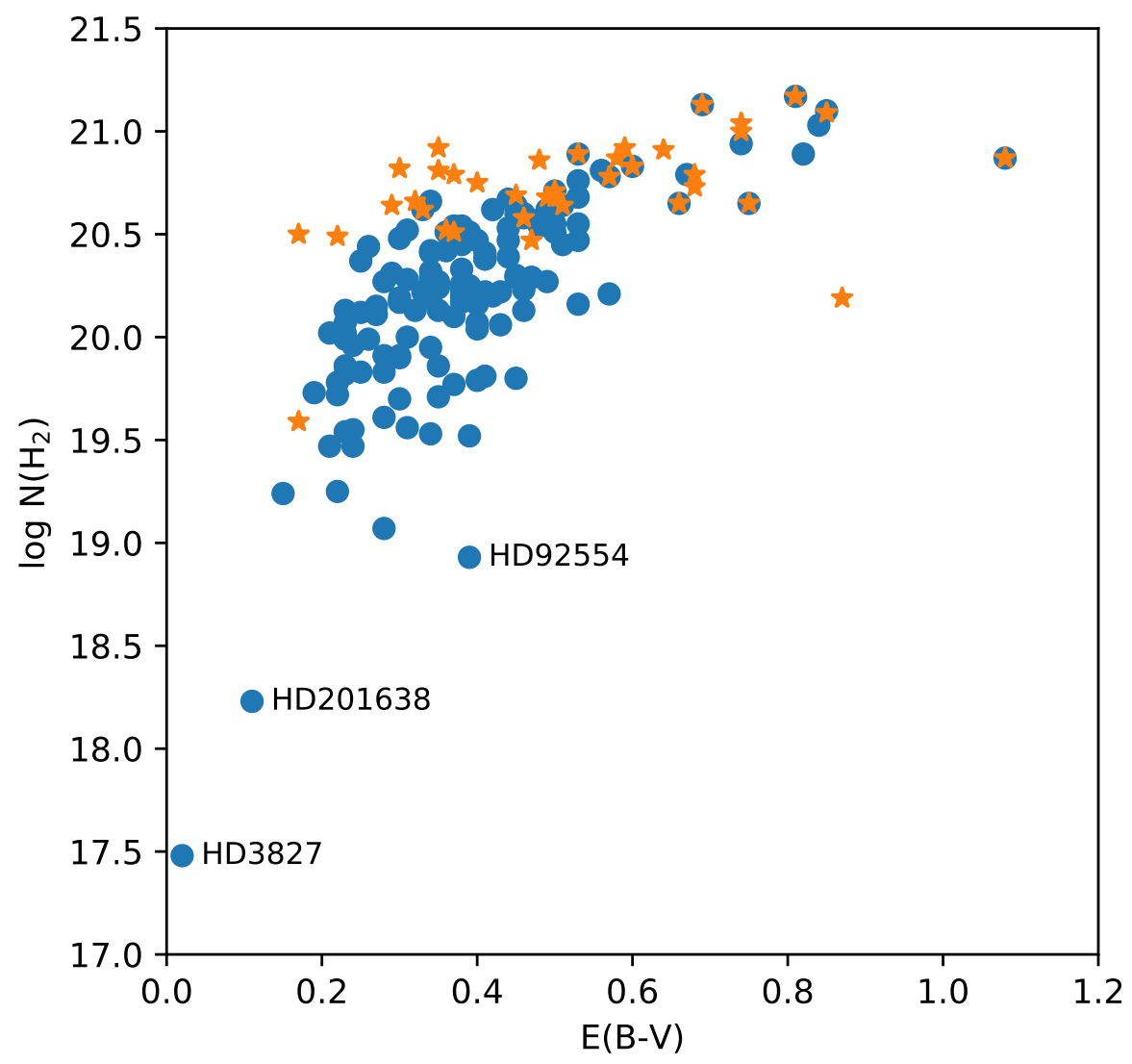

Fig. 3. - Distribution of $\mathrm{H}_{2}$ column density with color excess, labeling three sight lines with $\log N_{\mathrm{H} 2}<19.0$. Symbols are color-labeled for the current FUSE survey (blue circles) and translucent sight lines (orange stars) also studied by FUSE (Rachford et al. 2002, 2009). We re-analyzed 11 of these translucent sight lines with our $\mathrm{H}_{2}$ software for stars with new GOS photometry and SpTs (ID numbers 32, 82, 97, 98, 105, 107, $114,116,120,122,127)$. 

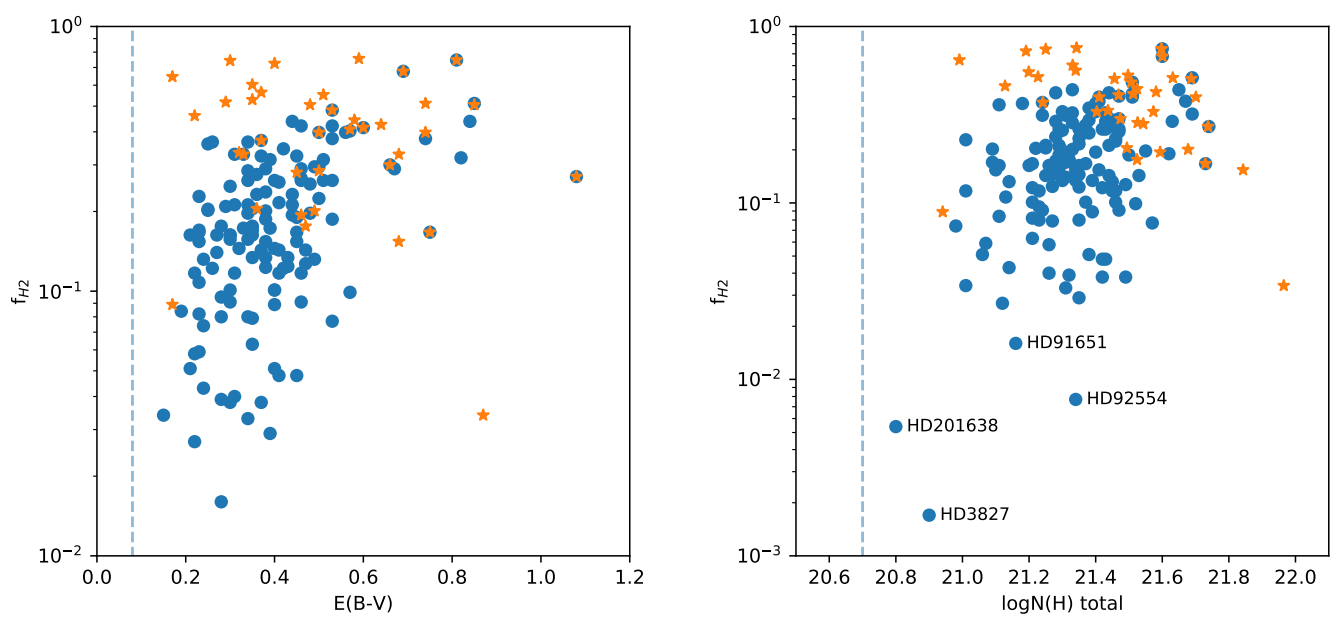

Fig. 4.- Molecular fraction $f_{\mathrm{H} 2}$ compared to color excess (left panel) and total hydrogen column density, $N_{\mathrm{H}}=N_{\mathrm{HI}}+2 N_{\mathrm{H} 2}$ (right panel). Vertical dashed lines show the transition to $f_{\mathrm{H} 2}>0.01$ at $E(B-V) \gtrsim 0.08$ and $\log N_{\mathrm{H}} \gtrsim 20.7$ seen in Copernicus data (Savage et al. 1977). Most of the FUSE targets are more distant and have $f_{\mathrm{H} 2}$ between $3 \%$ and $75 \%$. Symbols are color-coded as in Figure 3. The outlier (orange star) with $f_{\mathrm{H} 2}=0.034$ at $E(B-V)=0.87$ and $\log N_{\mathrm{H}}=21.96$ is the translucent sight line toward HD 164740 (Rachford et al. 2009).
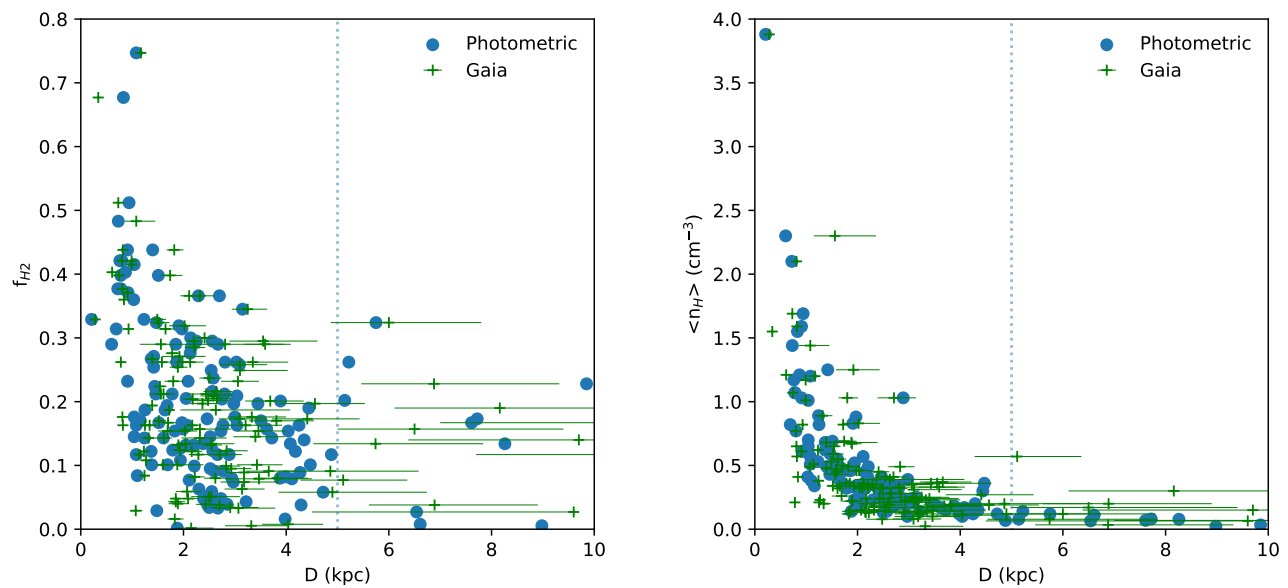

Fig. 5.- Molecular fraction $f_{\mathrm{H} 2}$ (left panel) and total hydrogen density $n_{\mathrm{H}}$ (right panel), averaged over photometric distance to target stars. Updated values (Shull \& Danforth 2019) are shown for both photometric and Gaia-DR2 parallax distances. For 129 stars with photometric distances $D \leq 5 \mathrm{kpc}$ (vertical dashed lines) the mean sight-line values are $\left\langle n_{\mathrm{HI}}\right\rangle=0.50 \mathrm{~cm}^{-3},\left\langle f_{\mathrm{H} 2}\right\rangle=0.20$, and $\left\langle N_{\mathrm{H}} / E(B-V)\right\rangle=6.07 \times$ $10^{21} \mathrm{~cm}^{-2} \mathrm{mag}^{-1}$. Table 5 lists these quantities for the full survey and for sub-samples $(D \leq 2 \mathrm{kpc}$ and $D \leq 5 \mathrm{kpc})$. 


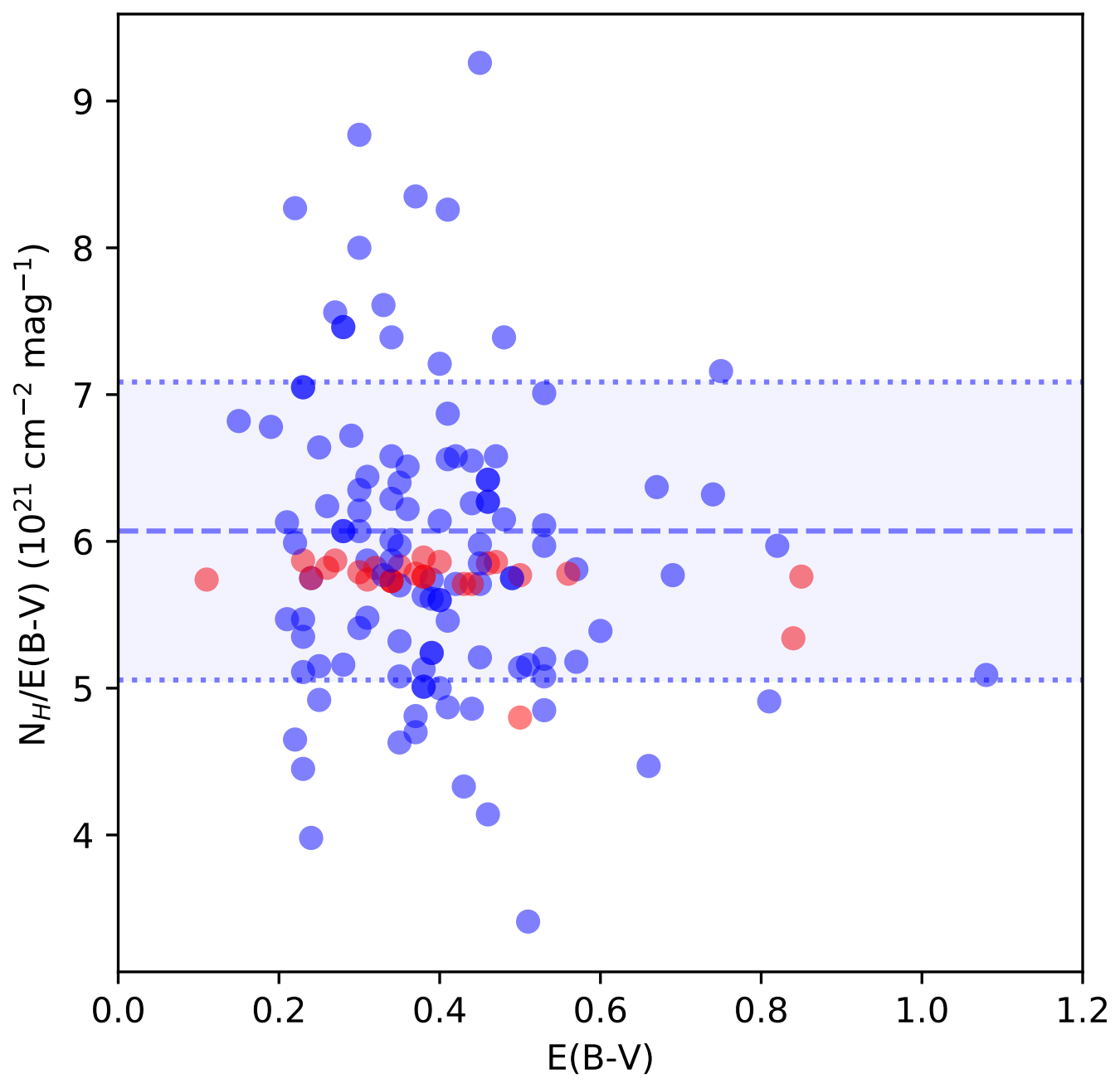

Fig. 6.- Distribution of the "gas-to-dust" ratio, $N_{H} / E(B-V)$, for 138 sight lines, in units of $10^{21}$ $\mathrm{cm}^{-2} \mathrm{mag}^{-1}$. One star (ID \#7, HD 3827) was omitted owing to its uncertain $E(B-V)$. Blue points are the 112 sight lines with $N_{\mathrm{HI}}$ determined from Ly $\alpha$ profile fits. Red points are the 26 stars lacking Ly $\alpha$ fits for H I (Tables 2 and 4), where $N_{\mathrm{HI}}$ was scaled from $E(B-V)$. The mean ratio for the 112 stars is $\left\langle N_{H} / E(B-V)\right\rangle=(6.07 \pm 1.01) \times 10^{21} \mathrm{~cm}^{-2} \mathrm{mag}^{-1}$. The mean and (rms) deviations are shown as horizontal lines and blue wash. 

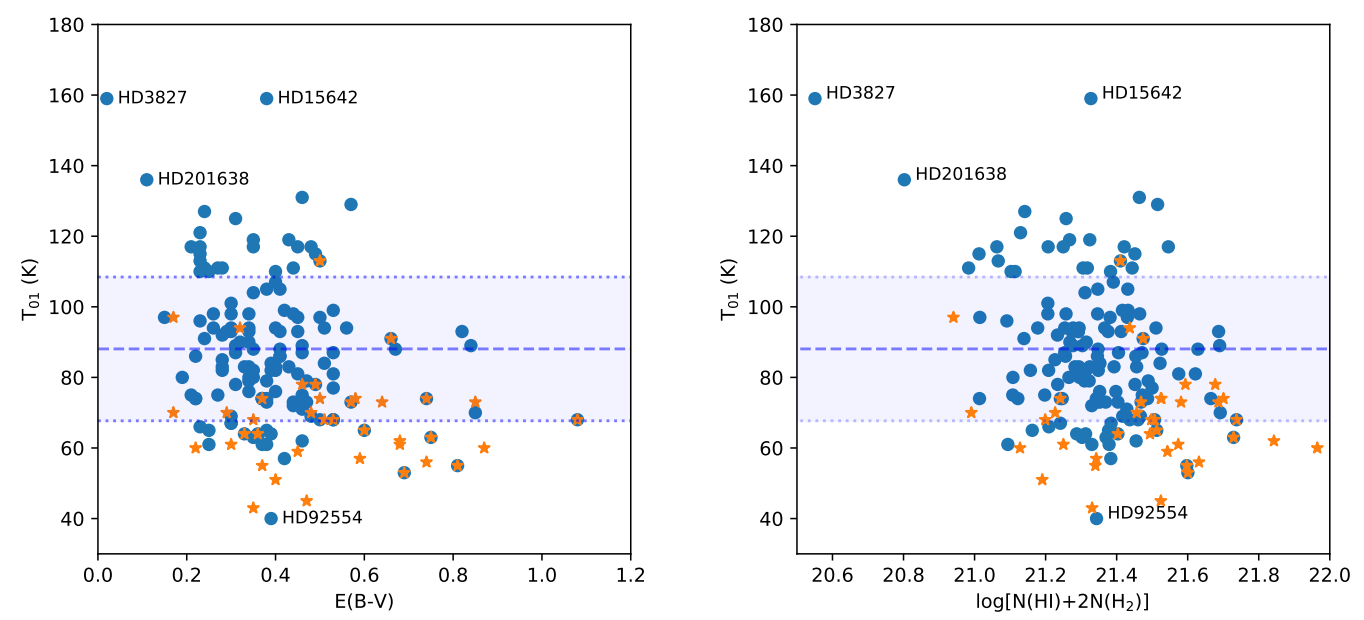

Fig. 7.- Rotational temperature $T_{01}$ vs. color excess (left panel) and total hydrogen column density (right panel). The mean value $\left\langle T_{01}\right\rangle=88 \pm 20 \mathrm{~K}$ (horizontal dashed line with $1 \sigma$ dispersions) should track the gas kinetic temperature in high-density clouds. Lower temperatures appear in translucent clouds at $E(B-V) \gtrsim 0.5$ and $\log N_{\mathrm{H}} \gtrsim 21.5$. Symbols are color-coded as in Figure 3 with four outlier targets labeled.
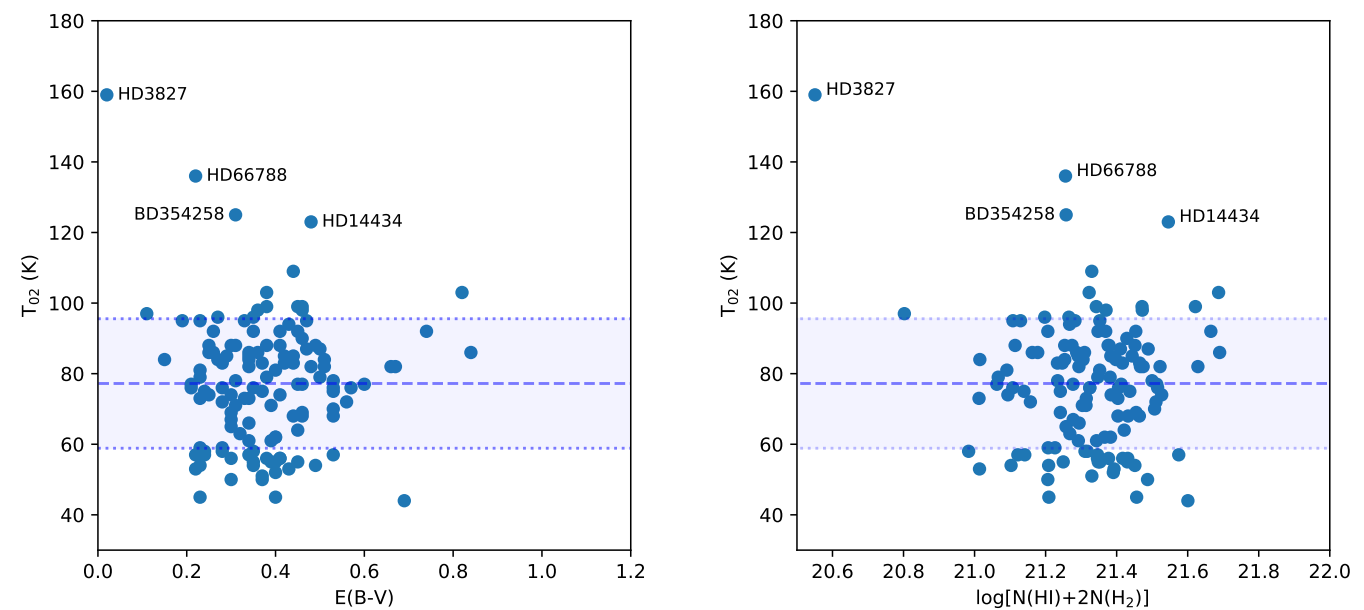

Fig. 8. - Rotational temperature $T_{02}$ vs. color excess (left panel) and total hydrogen column density (right panel). Four outlier targets are labeled. The other 128 stars have a mean value $\left\langle T_{02}\right\rangle=77 \pm 18 \mathrm{~K}$ (horizontal dashed lines with mean and $1 \sigma$ dispersions). Within individual sight-line errors (Section 3.4) and spreads of the distributions, $\left\langle T_{02}\right\rangle$ is similar to $\left\langle T_{01}\right\rangle=88 \pm 20 \mathrm{~K}$. The lowest three rotational levels $(J=0,1,2)$ are likely coupled to the gas kinetic temperature. 


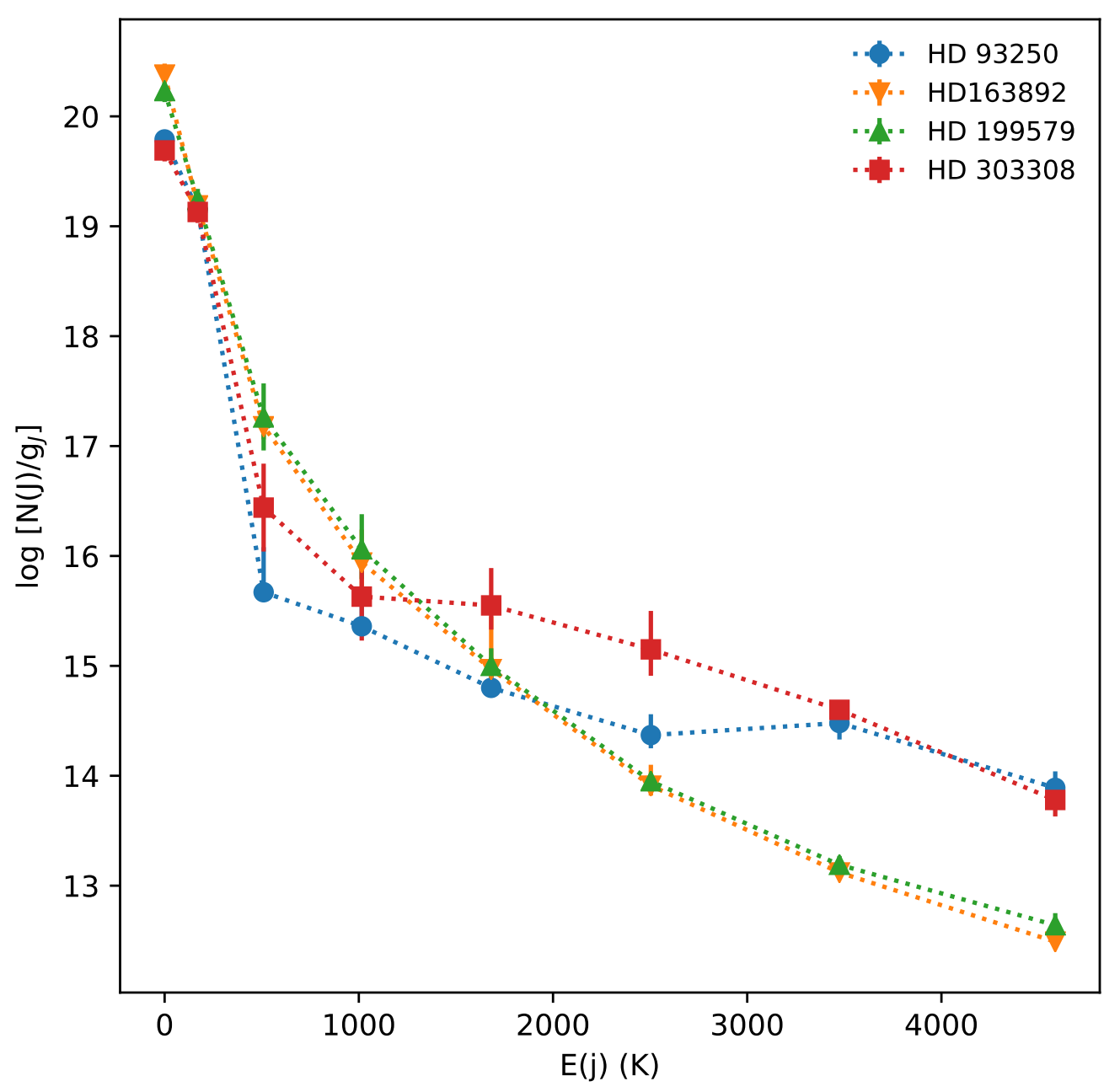

Fig. 9.- Populations of $\mathrm{H}_{2}$ rotational states, $\log \left[N(J) / g_{J}\right]$, vs. their excitation energies, $E(J) / k$, expressed as temperatures. The level statistical weights are $g_{J}=(2 S+1)(2 J+1)$, where $S=0$ (even- $\left.J\right)$ and $S=1$ (odd- $J$ ). We show distributions $(J=0-7)$ for four sight lines with different excitation temperatures of low- $J$ and high- $J$ states. The lowest levels $(J=0,1,2)$ appear thermally coupled. For HD $199579(\mathrm{O} 6.5 \mathrm{~V})$ we find $T_{01}=74 \mathrm{~K}$ and $T_{02}=75 \mathrm{~K}$, but $T_{24}=225 \mathrm{~K}$ and $T_{35}=307 \mathrm{~K}$. For HD $163892(\mathrm{O} 9 \mathrm{IV})$ we find $T_{01}=62 \mathrm{~K}$ and $T_{02}=69 \mathrm{~K}$, but $T_{24}=230 \mathrm{~K}$ and $T_{35}=319 \mathrm{~K}$. Two sight lines toward hotter stars show even higher excitation $\left(T_{24}=582 \mathrm{~K}, T_{35}=686 \mathrm{~K}\right)$ for HD $93250(\mathrm{O} 4 \mathrm{III})$ and $\left(T_{24}=569 \mathrm{~K}, T_{35}=1358 \mathrm{~K}\right)$ for HD $303308(\mathrm{O} 4.5 \mathrm{~V})$. 

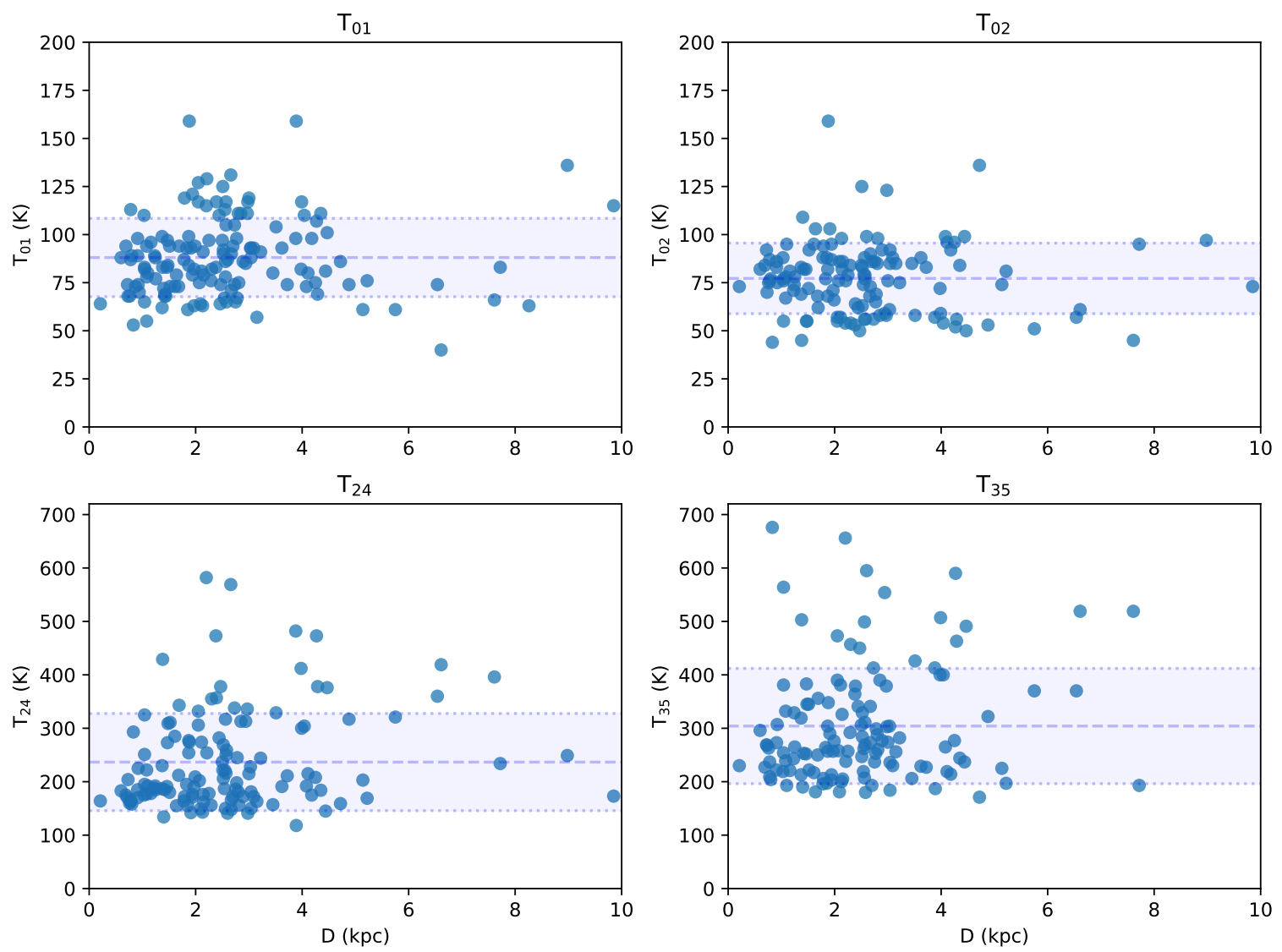

Fig. 10.- Excitation temperatures, $T_{01}$ and $T_{02}$, of the lowest rotational levels and $T_{24}$ and $T_{35}$ for higher excited levels, versus photometric distance to the target stars. Several sight lines with missing or uncertain values of $T_{02}, T_{24}, T_{35}$, have been omitted. Horizontal lines show means and $1 \sigma$ dispersions. A significant fraction (10-15\%) in the lower two panels have high values of $T_{24}$ and $T_{35}$, lying above the (rms) dispersions. 


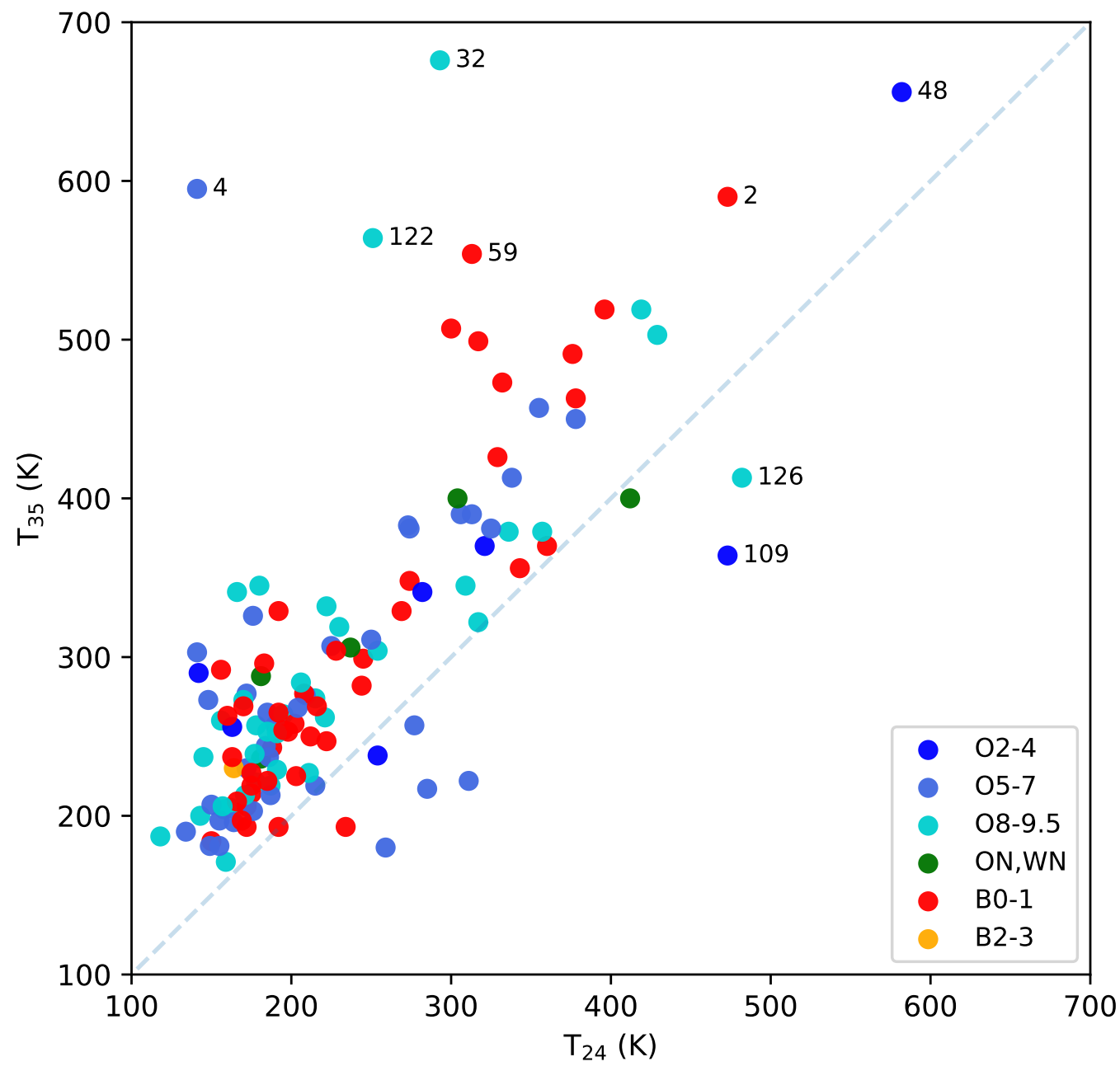

Fig. 11.- Relation between pairwise rotational excitation temperatures, $T_{35}$ and $T_{24}$, connecting ortho (odd$J$ ) and para (even- $J$ ) states. These two temperatures are correlated above $300 \mathrm{~K}$, usually with $T_{35}>T_{24}$. Most data points lie above the dashed line of slope unity. Stars of similar SpT are color-coded as follows: dark blue (O2-O4); cornflower blue (O5-O7); cyan (O8-O9.5); dark green (ON and WN); red (B0-B1); and orange (one B3 star). Using internal ID numbers, we label stars with high excitation temperatures, plus several that lie off the trend-line (see Section 3.5). Star \#137 (HD 303308, O4.5 Vfc) is not shown, with its off-scale excitation temperatures, $T_{35}=1358 \mathrm{~K}$ and $T_{24}=569 \mathrm{~K}$. 
Table 1. Stellar Parameters ${ }^{\mathrm{a}}$ and Distances ${ }^{\mathrm{b}}$

\begin{tabular}{|c|c|c|c|c|c|c|c|c|c|c|c|}
\hline ID & Target & $\begin{array}{c}\ell \\
(\mathrm{deg})\end{array}$ & $\begin{array}{c}b \\
(\mathrm{deg})\end{array}$ & $\begin{array}{c}B \\
(\mathrm{mag})\end{array}$ & $\begin{array}{c}V \\
(\mathrm{mag})\end{array}$ & $\begin{array}{c}E(B-V) \\
\quad(\mathrm{mag})\end{array}$ & $\mathrm{SpT}$ & $\begin{array}{l}D_{\text {phot }}{ }^{\mathrm{b}} \\
(\mathrm{kpc})\end{array}$ & $\begin{array}{l}D_{\mathrm{Gaia}}{ }^{\mathrm{b}} \\
(\mathrm{kpc})\end{array}$ & $\begin{array}{c}\text { Program } \\
\text { (FUSE ID) }\end{array}$ & $\begin{array}{c}t_{\exp } \\
(\mathrm{ksec})\end{array}$ \\
\hline 1 & BD $35^{\circ} 4258$ & 77.19 & -4.74 & 9.42 & 9.41 & 0.31 & B0.5 Vn & 2.51 & $1.89[1.71,2.10]$ & P1017902 & 5.673 \\
\hline 2 & $\mathrm{BD} 53^{\circ} 2820$ & 101.24 & -1.69 & 10.05 & 9.95 & 0.40 & Bo IVn & 4.27 & $3.18[2.82,3.65]$ & $\mathrm{P} 1223203$ & 5.147 \\
\hline 3 & $\mathrm{CPD}-59^{\circ} 2600$ & 287.60 & -0.74 & 8.82 & 8.61 & 0.53 & O6 Vf & 2.11 & $5.11[4.28,6.36]$ & P1221403 & 5.307 \\
\hline 4 & $\mathrm{CPD}-59^{\circ} 2603$ & 287.59 & -0.69 & 8.91 & 8.81 & 0.46 & $\mathrm{O} 7.5 \mathrm{~V}$ & 2.60 & $3.66[3.22,4.22]$ & S3040502 & 31.599 \\
\hline 5 & $\mathrm{CPD}-69^{\circ} 1743$ & 303.71 & -7.35 & 9.40 & 9.38 & 0.30 & B0.5 IIIn & 4.47 & $3.43[3.04,3.93]$ & P1013701 & 3.548 \\
\hline 6 & $\mathrm{CPD}-72^{\circ} 1184$ & 299.15 & -10.94 & 10.61 & 10.68 & 0.23 & B0 III & 9.85 & $6.88[5.46,9.32]$ & S5140103 & 9.172 \\
\hline 7 & HD 3827 & 120.79 & -23.23 & 7.76 & 8.01 & 0.02 & $\mathrm{~B} 0.7 \mathrm{~V}$ & 1.88 & $2.15[1.85,2.59]$ & $\mathrm{P} 1010302$ & 2.885 \\
\hline 8 & HD 5005A & 123.12 & -6.24 & 8.19 & 8.10 & 0.41 & O6.5 Vf & 2.89 & $2.71[2.39,3.14]$ & P1020102 & 6.372 \\
\hline 9 & HD 12323 & 132.91 & -5.87 & 8.88 & 8.90 & 0.29 & ON9 V & 3.04 & $2.59[2.33,2.93]$ & P1020201 & 3.865 \\
\hline 10 & HD 13268 & 133.96 & -4.99 & 8.31 & 8.18 & 0.44 & ON8.5 III & 2.80 & $1.61[1.50,1.74]$ & P1020304 & 4.430 \\
\hline 11 & HD 13745 & 134.58 & -4.96 & 7.99 & 7.83 & 0.46 & O9.7 IIn & 2.81 & $2.13[1.91,2.39]$ & P1020404 & 4.483 \\
\hline 12 & HD 14434 & 135.08 & -3.82 & 8.65 & 8.49 & 0.48 & O5.5 Vnfp & 2.98 & $2.37[2.13,2.67]$ & P1020504 & 4.426 \\
\hline 13 & HD 15137 & 137.46 & -7.58 & 7.91 & 7.86 & 0.35 & O9.5 II-IIIn & 3.00 & $3.33[2.86,4.00]$ & P1020602 & 2.282 \\
\hline 14 & HD $15558 \mathrm{~A}$ & 134.72 & +0.92 & 8.41 & 7.91 & 0.82 & O4.5 IIIf & 1.91 & $2.02[1.72,2.44]$ & P1170101 & 2.472 \\
\hline 15 & HD 15642 & 137.09 & -4.73 & 8.61 & 8.53 & 0.38 & O9.5 II-IIIn & 3.89 & $3.69[3.20,4.34]$ & P1020702 & 4.837 \\
\hline 16 & HD 34656 & 170.04 & +0.27 & 6.81 & 6.79 & 0.34 & O7.5 IIf & 1.99 & $2.32[1.92,2.93]$ & P1011301 & 4.179 \\
\hline 17 & HD 39680 & 194.07 & -5.88 & 8.01 & 7.99 & 0.34 & O6 Vnep & 2.67 & $3.07[2.57,3.79]$ & P1020901 & 3.866 \\
\hline 18 & HD 41161 & 164.97 & +12.89 & 6.68 & 6.76 & 0.23 & O8 Vn & 1.16 & $1.52[1.40,1.66]$ & $\mathrm{P} 1021002$ & 6.520 \\
\hline 19 & HD 42088 & 190.04 & +0.48 & 7.62 & 7.55 & 0.39 & O6 Vfz & 1.97 & $1.65[1.50,1.83]$ & P1021101 & 4.137 \\
\hline 20 & HD 45314 & 196.96 & +1.52 & 6.79 & 6.64 & 0.46 & O9 npe & 0.80 & $0.80[0.78,0.84]$ & P1021301 & 5.515 \\
\hline 21 & HD 46150 & 206.31 & -2.07 & 6.89 & 6.76 & 0.45 & $\mathrm{O} 5 \mathrm{Vf}$ & 1.47 & $1.52[1.36 .1 .73]$ & P1021401 & 4.858 \\
\hline 22 & HD 47360 & 207.33 & -0.79 & 8.32 & 8.19 & 0.41 & $B 0.5 \mathrm{~V}$ & 1.24 & $1.32[1.17,1.51]$ & P1021504 & 4.993 \\
\hline 23 & HD 47417 & 205.35 & +0.35 & 6.98 & 6.97 & 0.31 & B0 IV & 1.23 & $1.49[1.35,1.66]$ & P1021602 & 4.079 \\
\hline 24 & HD 60369 & 242.68 & -4.30 & 8.14 & 8.15 & 0.30 & O9 IV & 2.54 & $3.10[2.97,4.03]$ & P1050201 & 7.099 \\
\hline 25 & HD 61347 & 230.60 & +3.80 & 8.60 & 8.43 & 0.45 & O9 Ib & 4.44 & $8.16[6.11,12.3]$ & $\mathrm{P} 1022002$ & 7.290 \\
\hline 26 & HD 62866 & 237.48 & +1.80 & 9.08 & 9.01 & 0.35 & B0.5 IIIn & 3.51 & $3.81[3.28,4.54]$ & P1221004 & 4.614 \\
\hline 27 & HD 63005 & 242.50 & -0.93 & 9.08 & 9.13 & 0.27 & O7 Vf & 4.35 & $9.70[6.38,20.1]$ & P1022101 & 5.311 \\
\hline 28 & HD 64568 & 243.14 & +0.71 & 9.43 & 9.38 & 0.37 & O3 $\mathrm{Vf}^{*} \mathrm{z}$ & 5.75 & $6.00[4.87,7.80]$ & $\mathrm{P} 1221104$ & 4.154 \\
\hline 29 & HD 66695 & 245.01 & +2.21 & 9.77 & 9.78 & 0.27 & B0.5 IV & 4.25 & $3.21[2.72,3.92]$ & P1221201 & 8.080 \\
\hline 30 & HD 66788 & 245.43 & +2.05 & 9.36 & 9.45 & 0.22 & $\mathrm{O} 8 \mathrm{~V}$ & 4.72 & $4.90[3.85,6.74]$ & P1011801 & 4.209 \\
\hline 31 & HD 69106 & 254.52 & -1.33 & 7.03 & 7.13 & 0.19 & B $0.2 \mathrm{~V}$ & 1.10 & $1.24[1.17,1.33]$ & P1022302 & 0.404 \\
\hline 32 & HD 73882 & 260.18 & +0.64 & 7.60 & 7.22 & 0.69 & O8.5 IV & 0.83 & $0.34[0.30,0.41]$ & $\mathrm{P} 1161302$ & 13.627 \\
\hline 33 & HD 74194 & 264.04 & -1.95 & 7.78 & 7.57 & 0.50 & O8.5 Ib-II & 2.25 & $2.20[2.07,2.36]$ & P1022404 & 12.397 \\
\hline 34 & HD 74920 & 265.29 & -1.95 & 7.56 & 7.53 & 0.35 & O7.5 IV & 2.05 & $2.65[2.42,2.92]$ & P1022601 & 4.672 \\
\hline 35 & HD 89137 & 279.69 & +4.45 & 7.91 & 7.98 & 0.23 & ON9.7 IIn & 4.04 & $3.46[3.05,4.00]$ & P1022801 & 4.628 \\
\hline 36 & HD 90087 & 285.16 & -2.13 & 7.74 & 7.76 & 0.28 & O9.2 III & 2.52 & $2.93[2.62,3.32]$ & P1022901 & 3.934 \\
\hline 37 & HD 91597 & 286.86 & -2.37 & 9.88 & 9.84 & 0.30 & B1 IIIne & 4.29 & $6.89[5.61,8.90]$ & P1023004 & 6.228 \\
\hline 38 & HD 91651 & 286.55 & -1.72 & 8.82 & 8.84 & 0.28 & ON9.5 IIIn & 3.98 & $1.83[1.69,2.00]$ & P1023102 & 8.137 \\
\hline 39 & HD 91824 & 285.70 & +0.07 & 8.07 & 8.14 & 0.25 & $\mathrm{O} 7 \mathrm{Vfz}$ & 2.75 & $2.18[1.99,2.41]$ & A1180802 & 4.649 \\
\hline 40 & HD 92554 & 287.60 & -2.02 & 9.56 & 9.47 & 0.39 & O9.5 IIn & 6.61 & $4.04[3.53,4.72]$ & P1023202 & 6.051 \\
\hline 41 & HD 93028 & 287.64 & -1.19 & 8.24 & 8.30 & 0.24 & O9 IV & 2.97 & $3.18[2.72,3.83]$ & A1180902 & 4.446 \\
\hline 42 & HD $93129 A$ & 287.41 & -0.57 & 7.51 & 7.26 & 0.57 & $\mathrm{O} 2$ If* $^{*}+\mathrm{O} 3$ IIIf** & 2.21 & $2.83[2.60,3.11]$ & $\mathrm{P} 1170202$ & 7.361 \\
\hline 43 & HD 93146A & 287.67 & -1.05 & 8.48 & 8.45 & 0.35 & $\mathrm{O} 7 \mathrm{Vfz}$ & 2.30 & $3.14[2.80,3.58]$ & P1023301 & 4.140 \\
\hline 44 & HD 93204 & 287.57 & -0.71 & 8.57 & 8.48 & 0.41 & O5.5 Vf & 2.73 & $2.09[1.94,2.27]$ & P1023501 & 4.664 \\
\hline 45 & HD 93205 & 287.57 & -0.71 & 7.84 & 7.76 & 0.40 & $03.5 \mathrm{~V}$ & 2.44 & $2.49[2.27,2.76]$ & P1023601 & 4.693 \\
\hline 46 & HD 93206 & 287.67 & -0.94 & 6.40 & 6.31 & 0.39 & O9.7 Ibn & 1.48 & $1.07[0.96,1.20]$ & P1023401 & 4.140 \\
\hline 47 & HD 93222 & 287.74 & -1.02 & 8.15 & 8.10 & 0.37 & O7 IIIf & 2.47 & $2.70[2.47,2.99]$ & P1023701 & 3.920 \\
\hline 48 & HD 93250 & 287.51 & -0.54 & 7.58 & 7.41 & 0.49 & O4 IIIfc & 2.20 & $2.54[2.36,2.75]$ & P1023801 & 4.140 \\
\hline 49 & HD 93843 & 288.24 & -0.90 & 7.26 & 7.30 & 0.28 & O5 IIIf & 2.85 & $2.43[2.24,2.66]$ & P1024001 & 4.140 \\
\hline 50 & HD 96670 & 290.20 & +0.40 & 7.57 & 7.43 & 0.46 & O8 Ibf & 2.67 & $3.59[3.19,4.09]$ & P1024201 & 4.273 \\
\hline 51 & HD 96715 & 290.27 & +0.33 & 8.37 & 8.27 & 0.42 & $\mathrm{O} 4 \mathrm{Vf}$ & 3.15 & $3.25[2.94,3.63]$ & P1024301 & 4.597 \\
\hline 52 & HD 96917 & 289.28 & +3.06 & 7.15 & 7.07 & 0.39 & O8.5 Ibf & 2.46 & $4.41[3.72,5.43]$ & P1024401 & 7.982 \\
\hline 53 & HD 97471 & 290.36 & +1.62 & 9.55 & 9.30 & 0.30 & B0 V & 2.78 & $4.86[3.85,6.58]$ & A1180404 & 4.194 \\
\hline 54 & HD 97913 & 290.84 & +1.41 & 8.84 & 8.80 & 0.32 & B0.5 IVn & 2.52 & $3.40[2.97,3.97]$ & $\mathrm{P} 1221702$ & 9.961 \\
\hline
\end{tabular}


Table 1-Continued

\begin{tabular}{|c|c|c|c|c|c|c|c|c|c|c|c|}
\hline ID & Target & $\begin{array}{c}\ell \\
(\operatorname{deg})\end{array}$ & $\begin{array}{c}b \\
\text { (deg) }\end{array}$ & $\begin{array}{c}B \\
(\mathrm{mag})\end{array}$ & $\begin{array}{c}V \\
(\mathrm{mag})\end{array}$ & $\begin{array}{c}E(B-V) \\
\quad(\mathrm{mag})\end{array}$ & $\mathrm{SpT}$ & $\begin{array}{l}D_{\text {phot }}{ }^{\mathrm{b}} \\
(\mathrm{kpc})\end{array}$ & $\begin{array}{l}D_{\mathrm{Gaia}}{ }^{\mathrm{b}} \\
(\mathrm{kpc})\end{array}$ & $\begin{array}{c}\text { Program } \\
\text { (FUSE ID) }\end{array}$ & $\begin{array}{c}t_{\exp } \\
(\mathrm{ksec})\end{array}$ \\
\hline 55 & HD 99857 & 294.78 & -4.94 & 7.56 & 7.45 & 0.35 & B0.5 Ib & 3.04 & $2.17[2.04,2.33]$ & P1024501 & 4.266 \\
\hline 56 & HD 99890 & 291.75 & +4.43 & 8.22 & 8.26 & 0.24 & B0 IIIn & 3.22 & $1.85[1.70,2.03]$ & P1024601 & 4.584 \\
\hline 57 & HD 100199 & 293.94 & -1.49 & 8.14 & 8.14 & 0.30 & B0 IIIne & 2.77 & $1.25[1.15,1.37]$ & P1221801 & 4.623 \\
\hline 58 & HD 100213 & 294.81 & -4.14 & 8.25 & 8.22 & 0.34 & $\mathrm{O} 8 \mathrm{Vn}$ & 2.15 & $2.21[2.03,2.42]$ & P1024701 & 4.561 \\
\hline 59 & HD 100276 & 293.31 & +0.77 & 7.20 & 7.16 & 0.28 & B0.5 Ib & 2.94 & $2.62[2.40,2.89]$ & P1024801 & 4.125 \\
\hline 60 & HD 101131 & 294.78 & -1.62 & 7.14 & 7.15 & 0.31 & O5.5 Vf & 1.78 & $2.44[2.07,2.97]$ & P1024901 & 4.302 \\
\hline 61 & HD 101190 & 294.78 & -1.49 & 7.31 & 7.27 & 0.36 & O6 IVf & 2.09 & $3.06[2.73,3.47]$ & P1025001 & 3.392 \\
\hline 62 & HD 101205 & 294.85 & -1.65 & 6.48 & 6.42 & 0.38 & O7 II: & 1.64 & $2.63[1.94,4.08]$ & P1025101 & 0.059 \\
\hline 63 & HD 101298 & 294.94 & -1.69 & 8.11 & 8.05 & 0.38 & O6 IVf & 2.58 & $2.50[2.31,2.74]$ & P1025201 & 4.565 \\
\hline 64 & HD 101413 & 295.03 & -1.71 & 8.40 & 8.35 & 0.36 & $\mathrm{O} 8 \mathrm{~V}$ & 2.13 & $1.78[1.65,1.94]$ & P1025301 & 4.161 \\
\hline 65 & HD 101436 & 295.04 & -1.71 & 7.62 & 7.56 & 0.38 & $06.5 \mathrm{~V}$ & 1.85 & $2.81[2.18,3.95]$ & P1025401 & 4.152 \\
\hline 66 & HD 103779 & 296.85 & -1.02 & 7.19 & 7.20 & 0.23 & B0.5 Iab & 3.99 & $2.22[2.06,2.41]$ & P1025601 & 4.336 \\
\hline 67 & HD 104705 & 297.45 & -0.34 & 7.74 & 7.76 & 0.26 & B0 Ib & 4.18 & $2.16[1.99,2.36]$ & P1025701 & 4.482 \\
\hline 68 & HD 115071 & 305.76 & +0.15 & 8.15 & 7.94 & 0.51 & O9.5 III & 1.87 & $1.98[1.84,2.14]$ & P1025901 & 4.347 \\
\hline 69 & HD 116781 & 307.05 & -0.07 & 7.73 & 7.60 & 0.43 & B0 IIIne & 1.79 & $1.93[1.77,2.12]$ & P1026101 & 2.961 \\
\hline 70 & HD 116852 & 304.88 & -16.13 & 8.38 & 8.47 & 0.22 & O8.5 II-IIIf & 4.88 & $13[7.7,18]$ & P1013801 & 7.212 \\
\hline 71 & HD 118571 & 308.70 & +1.35 & 8.74 & 8.76 & 0.26 & B0.5 IVn & 2.70 & $2.32[2.09,2.60]$ & P1222001 & 4.434 \\
\hline 72 & HD $124314 \mathrm{~A}$ & 312.67 & -0.42 & 6.85 & 6.64 & 0.53 & O6 IVnf & 1.25 & $1.72[1.62,1.83]$ & P1026201 & 4.364 \\
\hline 73 & HD 124979 & 316.40 & +9.08 & 8.62 & 8.53 & 0.41 & O7.5 IVn & 3.09 & $3.10[2.71,3.63]$ & P1026301 & 5.105 \\
\hline 74 & HD 148422 & 329.92 & -5.60 & 8.69 & 8.60 & 0.35 & B1 Ia & 8.26 & $5.74[4.53,7.84]$ & P1015003 & 3.242 \\
\hline 75 & HD 152218 & 343.53 & +1.28 & 7.78 & 7.61 & 0.48 & O9 IVn & 1.42 & $1.89[1.73,2.08]$ & P1015402 & 9.486 \\
\hline 76 & HD 152233 & 343.48 & +1.22 & 6.72 & 6.59 & 0.45 & O6 IIf & 1.52 & $1.70[1.57,1.86]$ & P1026702 & 4.104 \\
\hline 77 & HD 152248 & 343.46 & +1.18 & 6.25 & 6.10 & 0.47 & O7 Iabf & 1.61 & $1.62[1.48,1.78]$ & P1026801 & 4.445 \\
\hline 78 & HD 152314 & 343.52 & +1.14 & 8.05 & 7.86 & 0.50 & O9 IV & 1.44 & $1.54[1.42,1.67]$ & P1026901 & 4.040 \\
\hline 79 & HD 152623 & 344.62 & +1.61 & 6.75 & 6.67 & 0.40 & O7 Vnf & 1.04 & data problem & P1027001 & 6.056 \\
\hline 80 & HD 152723 & 344.81 & +1.61 & 7.31 & 7.16 & 0.47 & O6.5 III & 1.94 & data problem & P1027102 & 5.019 \\
\hline 81 & HD 153426 & 347.14 & +2.38 & 7.71 & 7.47 & 0.45 & O8.5 III & 1.83 & $2.03[1.84,2.27]$ & P1027202 & 5.413 \\
\hline 82 & HD 154368 & 349.97 & +3.22 & 6.65 & 6.13 & 0.81 & O9.2 Iab & 1.08 & $1.17[1.11,1.25]$ & P1161901 & 12.320 \\
\hline 83 & HD 156292 & 345.35 & -3.08 & 7.75 & 7.49 & 0.56 & O9.7 III & 1.51 & $1.74[1.55,1.98]$ & P1027403 & 6.648 \\
\hline 84 & HD 157857 & 12.97 & +13.5 & 7.95 & 7.78 & 0.49 & O6.5 IIIf & 2.56 & $3.55[2.89,4.61]$ & P1027501 & 4.022 \\
\hline 85 & HD 158661 & 8.29 & +9.05 & 8.32 & 8.18 & 0.38 & B0.5 Ib & 4.08 & $2.55[2.23,2.99]$ & P1222201 & 6.597 \\
\hline 86 & HD 161807 & 351.78 & -5.85 & 6.92 & 6.99 & 0.23 & O9.7 III:n & 1.94 & $1.27[1.18,1.37]$ & P1222302 & 1.906 \\
\hline 87 & HD 163758 & 355.36 & -6.10 & 7.35 & 7.32 & 0.35 & O6.5 Iafp & 4.11 & $3.47[2.82,4.50]$ & P1015901 & 5.383 \\
\hline 88 & HD 163892 & 7.15 & +0.62 & 7.60 & 7.44 & 0.46 & O9 IVn & 1.37 & $1.39[1.28,1.52]$ & P1027602 & 6.583 \\
\hline 89 & HD 164816 & 6.06 & -1.20 & 7.09 & 7.08 & 0.31 & $09.5 \mathrm{~V}$ & 1.08 & $1.14[1.06,1.24]$ & P1016001 & 5.043 \\
\hline 90 & HD 165052 & 6.12 & -1.48 & 6.96 & 6.86 & 0.42 & O5.5 Vz & 1.37 & $1.23[1.16,1.30]$ & P1027801 & 4.874 \\
\hline 91 & HD 165246 & 6.40 & -1.56 & 7.80 & 7.71 & 0.40 & $\mathrm{O} 8 \mathrm{Vn}$ & 1.38 & $1.88[1.55,2.39]$ & P1050301 & 7.782 \\
\hline 92 & HD 166546 & 10.36 & -0.92 & 7.26 & 7.22 & 0.34 & O9.5 IV & 1.46 & $1.56[1.35,1.84]$ & P1222501 & 3.444 \\
\hline 93 & HD 166716 & 14.85 & +1.39 & 7.87 & 7.95 & 0.38 & B0 II-III & 2.73 & $1.69[1.50,1.92]$ & P1050401 & 5.788 \\
\hline 94 & HD 167402 & 2.26 & -6.39 & 8.94 & 8.95 & 0.23 & B0 Ib & 7.61 & $<14 \mathrm{kpc}$ & P1016201 & 3.856 \\
\hline 95 & HD 167659 & 12.20 & -1.27 & 7.60 & 7.39 & 0.53 & O7 II-IIIf & 1.87 & $1.58[1.40,1.80]$ & P1028001 & 5.816 \\
\hline 96 & HD 167771 & 12.70 & -1.13 & 6.66 & 6.54 & 0.44 & O7 IIInf & 1.40 & $1.82[1.67,2.00]$ & P1028101 & 3.836 \\
\hline 97 & HD 167971 & 18.25 & +1.68 & 8.27 & 7.50 & 1.08 & O8 Iafn & 1.42 & $1.92[1.58,2.43]$ & P1162101 & 9.450 \\
\hline 98 & HD 168076 & 16.84 & +0.84 & 8.61 & 8.18 & 0.75 & O4 IIIf & 1.97 & data problem & P1162201 & 6.601 \\
\hline 99 & HD 168941 & 5.82 & -6.31 & 9.41 & 9.34 & 0.37 & O9.5 IVp & 3.72 & $2.32[1.96,2.84]$ & P1016502 & 3.983 \\
\hline 100 & HD 172140 & 5.28 & -10.61 & 9.90 & 9.96 & 0.22 & B0.5 III & 6.54 & $<9.6 \mathrm{kpc}$ & P1016603 & 5.244 \\
\hline 101 & HD 175754 & 16.39 & -9.92 & 6.93 & 7.01 & 0.23 & O8 IInfp & 2.55 & $2.07[1.85,2.35]$ & P1016802 & 1.932 \\
\hline 102 & HD 175876 & 15.28 & -10.58 & 6.81 & 6.92 & 0.21 & O6.5 IIInf & 2.57 & $2.54[2.15,3.10]$ & P1016902 & 1.932 \\
\hline 103 & HD 177989 & 17.81 & -11.88 & 9.28 & 9.33 & 0.25 & B0 III & 5.14 & $2.48[2.17,2.87]$ & P1017101 & 10.289 \\
\hline 104 & HD 178487 & 25.78 & -8.56 & 8.82 & 8.66 & 0.40 & B0 Ib & 5.22 & $3.35[2.87,4.04]$ & P1017201 & 8.902 \\
\hline 105 & HD 179406 & 28.23 & -8.31 & 5.47 & 5.34 & 0.33 & B3 IV & 0.21 & $0.28[0.27,0.29]$ & E0610301 & 21.228 \\
\hline 106 & HD 179407 & 24.02 & -10.40 & 9.50 & 9.41 & 0.33 & B0.5 Ib & 7.72 & $3.09[2.69,3.63]$ & P1017301 & 13.858 \\
\hline 107 & HD 185418 & 53.60 & -2.17 & 7.67 & 7.45 & 0.50 & B $0.5 \mathrm{~V}$ & 0.78 & $0.74[0.72,0.76]$ & P1162301 & 4.399 \\
\hline 108 & HD 187459 & 68.81 & +3.85 & 6.64 & 6.49 & 0.44 & B0.5 Ib & 1.68 & $1.39[1.31,1.48]$ & P1028201 & 4.504 \\
\hline
\end{tabular}


Table 1 - Continued

\begin{tabular}{|c|c|c|c|c|c|c|c|c|c|c|c|}
\hline ID & Target & $\begin{array}{c}\ell \\
(\mathrm{deg})\end{array}$ & $\begin{array}{c}b \\
(\mathrm{deg})\end{array}$ & $\begin{array}{c}B \\
(\mathrm{mag})\end{array}$ & $\begin{array}{c}V \\
(\mathrm{mag})\end{array}$ & $\begin{array}{c}E(B-V) \\
(\mathrm{mag})\end{array}$ & $\mathrm{SpT}$ & $\begin{array}{l}D_{\text {phot }}{ }^{\mathrm{b}} \\
(\mathrm{kpc})\end{array}$ & $\begin{array}{l}D_{\mathrm{Gaia}}^{\mathrm{b}} \\
(\mathrm{kpc})\end{array}$ & $\begin{array}{c}\text { Program } \\
\text { (FUSE ID) }\end{array}$ & $\begin{array}{c}t_{\exp } \\
(\mathrm{ksec})\end{array}$ \\
\hline 109 & HD 190429A & 72.59 & +2.61 & 7.20 & 7.09 & 0.43 & O4 If & 2.38 & $2.04[1.91,2.20]$ & P1028401 & 5.390 \\
\hline 110 & HD 190918 & 72.65 & +2.07 & 6.88 & 6.75 & 0.45 & O9.5Iab+WN4 & 2.39 & $1.85[1.74,1.97]$ & P1028501 & 5.510 \\
\hline 111 & HD 191495 & 72.74 & +1.41 & 8.51 & 8.41 & 0.40 & Bo IV-V & 1.69 & $1.60[1.48,1.74]$ & P1222901 & 6.329 \\
\hline 112 & HD 191877 & 61.57 & -6.45 & 6.27 & 6.28 & 0.21 & $\mathrm{~B} 1 \mathrm{Ib}$ & 2.07 & $1.34[1.23,1.48]$ & P1028701 & 6.132 \\
\hline 113 & HD 192035 & 83.33 & +7.76 & 8.26 & 8.22 & 0.34 & B0 III-IVn & 2.29 & $2.11[1.94,2.31]$ & P1028603 & 6.624 \\
\hline 114 & HD 192639 & 74.90 & +1.48 & 7.46 & 7.11 & 0.66 & O7.5 Iab & 2.14 & $2.41[2.23,2.62]$ & P1162401 & 4.834 \\
\hline 115 & HD 195965 & 85.71 & +5.00 & 6.93 & 6.98 & 0.25 & B0 V & 1.03 & $0.84[0.81,0.87]$ & P1028803 & 14.047 \\
\hline 116 & HD 199579 & 85.70 & -0.30 & 6.01 & 5.96 & 0.37 & O6.5 Vfz & 0.92 & $0.91[0.87,0.97]$ & P1162501 & 4.307 \\
\hline 117 & HD 201345 & 78.44 & -9.54 & 7.61 & 7.75 & 0.15 & ON9.2 IV & 2.50 & $2.91[2.51,3.46]$ & P1223001 & 5.104 \\
\hline 118 & HD 201638 & 80.29 & -8.45 & 8.92 & 9.05 & 0.11 & $\mathrm{~B} 0.5 \mathrm{Ib}$ & 8.98 & $3.32[2.81,4.06]$ & P1018001 & 4.920 \\
\hline 119 & HD $203374 \mathrm{~A}$ & 100.51 & +8.62 & 6.91 & 6.67 & 0.53 & B0 IVpe & 0.78 & uncertain & B0300102 & 10.170 \\
\hline 120 & HD 206267 & 99.29 & +3.74 & 5.82 & 5.62 & 0.53 & O6 Vf & 0.73 & $1.08[0.86,1.45]$ & P1162701 & 4.872 \\
\hline 121 & HD 206773 & 99.80 & +3.62 & 7.12 & 6.91 & 0.51 & B0 Vpe & 0.69 & $0.93[0.91,0.96]$ & B0710901 & 4.399 \\
\hline 122 & HD 207198 & 103.14 & +6.99 & 6.27 & 5.96 & 0.60 & O8.5 II & 1.04 & $0.99[0.94,1.05]$ & P1162801 & 13.177 \\
\hline 123 & HD 207308 & 103.11 & +6.82 & 7.74 & 7.49 & 0.53 & $\mathrm{~B} 0.5 \mathrm{~V}$ & 0.76 & $0.99[0.96,1.03]$ & B0300301 & 22.382 \\
\hline 124 & HD 208440 & 104.03 & +6.44 & 7.93 & 7.91 & 0.28 & $\mathrm{~B} 1 \mathrm{~V}$ & 1.04 & $0.81[0.79,0.83]$ & B0300401 & 9.777 \\
\hline 125 & HD 209339 & 104.58 & +5.87 & 6.73 & 6.73 & 0.30 & O9.7 IV & 1.08 & $0.82[0.80,0.85]$ & B0300501 & 2.022 \\
\hline 126 & HD 210809 & 99.85 & -3.13 & 7.59 & 7.54 & 0.34 & O9 Iab & 3.88 & $3.83[3.37,4.42]$ & P1223103 & 10.065 \\
\hline 127 & HD 210839 & 103.83 & +2.61 & 5.30 & 5.05 & 0.57 & O6.5 Infp & 0.87 & $0.61[0.56,0.66]$ & P1163101 & 6.045 \\
\hline 128 & HD 216044 & 105.93 & -3.64 & 8.59 & 8.51 & 0.38 & B0 III-IV & 2.57 & $2.84[2.53,3.25]$ & P1223801 & 5.612 \\
\hline 129 & HD 216532 & 109.65 & +2.68 & 8.54 & 8.00 & 0.85 & O8.5 Vn & 0.94 & $0.73[0.72,0.75]$ & A0510202 & 22.736 \\
\hline 130 & HD 216898 & 109.93 & +2.39 & 8.53 & 8.00 & 0.84 & O9 V & 0.91 & $0.82[0.80,0.84]$ & A0510303 & 19.543 \\
\hline 131 & HD 217035 & 110.25 & +2.86 & 8.20 & 7.74 & 0.74 & B0.5 V & 0.72 & $0.81[0.79,0.83]$ & A0510404 & 10.187 \\
\hline 132 & HD 217312 & 110.56 & +2.95 & 7.81 & 7.42 & 0.67 & B0.5 V & 0.60 & $1.56[1.15,2.36]$ & P1930501 & 10.814 \\
\hline 133 & HD 218915 & 108.06 & -6.89 & 7.22 & 7.20 & 0.30 & O9.2 Iab & 3.62 & $6.5[5.0,9.4]$ & P1018801 & 5.325 \\
\hline 134 & HD 224151 & 115.44 & -4.64 & 6.01 & 6.19 & 0.44 & B0.5 II-III & 0.91 & $1.80[1.63,2.01]$ & P1224103 & 6.145 \\
\hline 135 & HD 224257 & 115.25 & -6.06 & 7.92 & 7.98 & 0.24 & B0.2 IV & 2.05 & $1.90[1.75,2.08]$ & P1050601 & 7.032 \\
\hline 136 & HD 224868 & 116.87 & -1.44 & 7.39 & 7.29 & 0.34 & B0 Ib & 3.03 & $0.78[0.73,0.83]$ & P1220201 & 5.105 \\
\hline 137 & HD 303308 & 287.59 & -0.61 & 8.33 & 8.19 & 0.46 & $\mathrm{O} 4.5 \mathrm{Vfc}$ & 2.66 & $2.29[2.10,2.51]$ & P1221602 & 7.679 \\
\hline 138 & HD 308813 & 294.79 & -1.61 & 9.32 & 9.28 & 0.34 & O9.7 IVn & 3.45 & $4.56[3.87,5.53]$ & P1221903 & 5.667 \\
\hline 139 & HD 332407 & 64.28 & +3.11 & 8.63 & 8.50 & 0.41 & B0.5 III & 2.56 & $2.64[2.39,2.95]$ & P1222802 & 4.277 \\
\hline
\end{tabular}

${ }^{a}$ Updated stellar parameters, photometry, spectral types, and distances are from a survey of 139 OB-type stars (Shull \& Danforth 2019) with their internal target ID (column 1). These parameters are based on optical and near-IR digital photometry and extinction corrections from the Galactic O-Star Spectroscopic Survey (Maíz Apellániz et al. 2004) and new spectral types (Sota et al. 2011, 2014). Columns 3 and 4 list the Galactic longitude and latitude. Later columns list the stellar photometry $[B, V, E(B-V)]$, spectral types (SpT), photometric distances $\left(D_{\text {phot }}\right)$, and parallax distances $\left(D_{\text {Gaia }}\right)$ listed in Tables 1 and 2 of Shull \& Danforth (2019). The last two columns give the Program ID and exposure time of the primary FUSE spectroscopic observations.

${ }^{\mathrm{b}}$ Photometric distances $\left(D_{\text {phot }}\right.$ ) are based on GOS photometry, extinctions, and SpTs and a new set of absolute magnitudes (see Shull \& Danforth 2019). When GOS data were not available, we based photometric distances (shown in boldface) on photometry and SpT in the literature. Parallax distances $\left(D_{\text {Gaia }}\right)$ and error ranges are based on parallaxes and errors from the Gaia-DR2 archive, after adding a constant parallax offset of 0.03 mas. 
Table 2. H I Column Density Measurements ${ }^{\mathrm{a}}$

\begin{tabular}{|c|c|c|c|c|c|c|c|}
\hline ID & $\begin{array}{l}\text { Target } \\
\text { (Star) }\end{array}$ & $\begin{array}{l}E_{B-V^{\mathrm{b}}} \\
(\mathrm{mag})\end{array}$ & $\begin{array}{c}\log N_{\mathrm{HI}}^{\mathrm{c}} \\
\text { (Adopted) }\end{array}$ & $\begin{array}{l}\log N_{\mathrm{HI}} \\
(\mathrm{SVS} 85)\end{array}$ & $\begin{array}{c}\log N_{\mathrm{HI}} \\
(\mathrm{DS} 94)\end{array}$ & $\begin{array}{c}\log N_{\mathrm{HI}} \\
(\mathrm{J} 19)\end{array}$ & $\begin{array}{l}\log N_{\mathrm{HI}}{ }^{c} \\
\text { (Other) }\end{array}$ \\
\hline 1 & BD $35^{\circ} 4258$ & 0.31 & 21.24 & & & $21.24_{-0.07}^{+0.03}$ & \\
\hline 2 & $\mathrm{BD} 53^{\circ} 2820$ & 0.40 & 21.35 & & & $21.35_{-0.07}^{+0.05}$ & \\
\hline 3 & $\mathrm{CPD}-59^{\circ} 2600$ & 0.53 & 21.54 & $21.48 \pm 0.10$ & $21.54 \pm 0.07$ & & $21.48 \pm 0.15(\mathrm{FM} 90)$ \\
\hline 4 & $\mathrm{CPD}-59^{\circ} 2603$ & 0.46 & 21.43 & $21.48 \pm 0.15$ & $21.46 \pm 0.07$ & $21.43_{-0.05}^{+0.04}$ & \\
\hline 5 & $\mathrm{CPD}-69^{\circ} 1743$ & 0.30 & 21.16 & & $21.12 \pm 0.11$ & $21.16_{-0.09}^{+0.04}$ & \\
\hline 6 & $\mathrm{CPD}-72^{\circ} 1184$ & 0.23 & 20.90 & & $20.90 \pm 0.08$ & & \\
\hline 7 & HD 3827 & 0.02 & 20.55 & & $20.56 \pm 0.09$ & $20.55_{-0.05}^{+0.07}$ & \\
\hline 8 & $\mathrm{HD} 5005 \mathrm{~A}$ & 0.41 & 21.40 & $21.48 \pm 0.15$ & $21.40 \pm 0.10$ & & \\
\hline 9 & HD 12323 & 0.29 & 21.19 & & & $21.19_{-0.05}^{+0.04}$ & \\
\hline 10 & HD 13268 & 0.44 & 21.34 & & $21.34 \pm 0.18$ & $21.34_{-0.07}^{+0.07}$ & \\
\hline 11 & HD 13745 & 0.46 & 21.34 & & $21.26 \pm 0.10$ & $21.34_{-0.05}^{+0.05}$ & \\
\hline 12 & HD 14434 & 0.48 & 21.45 & & $21.45 \pm 0.08$ & & \\
\hline 13 & HD 15137 & 0.35 & 21.24 & & $21.11 \pm 0.16$ & $21.24_{-0.08}^{+0.08}$ & \\
\hline 14 & HD $15558 \mathrm{~A}$ & 0.82 & 21.52 & & $21.52 \pm 0.18$ & & \\
\hline 15 & HD 15642 & 0.38 & 21.23 & $21.18 \pm 0.07$ & $21.23 \pm 0.08$ & & \\
\hline 16 & HD 34656 & 0.34 & 21.22 & & & & 21.22 (scaled) \\
\hline 17 & HD 39680 & 0.34 & 21.30 & & $21.30 \pm 0.08$ & & \\
\hline 18 & HD 41161 & 0.23 & 21.01 & $20.98 \pm 0.07$ & $21.01 \pm 0.08$ & $21.09_{-0.05}^{+0.05}$ & \\
\hline 19 & HD 42088 & 0.39 & 21.15 & & $21.15 \pm 0.08$ & & \\
\hline 20 & HD 45314 & 0.46 & 21.04 & & $21.04 \pm 0.09$ & & \\
\hline 21 & HD 46150 & 0.45 & 21.26 & $21.20 \pm 0.10$ & $21.26 \pm 0.12$ & & \\
\hline 22 & HD 47360 & 0.41 & 21.46 & & $21.46 \pm 0.08$ & & \\
\hline 23 & HD 47417 & 0.31 & 21.13 & $21.20 \pm 0.05$ & $21.13 \pm 0.09$ & & \\
\hline 24 & HD 60369 & 0.30 & 21.26 & & $21.26 \pm 0.10$ & & \\
\hline 25 & HD 61347 & 0.45 & 21.53 & & $21.53 \pm 0.13$ & & \\
\hline 26 & HD 62866 & 0.35 & 21.23 & & & & 21.23 (scaled) \\
\hline 27 & HD 63005 & 0.27 & 21.24 & & & $21.24_{-0.06}^{+0.03}$ & \\
\hline 28 & HD 64568 & 0.37 & 21.16 & & & & 21.16 (scaled) \\
\hline 29 & HD 66695 & 0.27 & 21.12 & & & & 21.12 (scaled) \\
\hline 30 & HD 66788 & 0.22 & 21.23 & & & $21.23_{-0.02}^{+0.04}$ & \\
\hline 31 & HD 69106 & 0.19 & 21.07 & & $21.08 \pm 0.06$ & $21.07_{-0.04}^{+0.02}$ & \\
\hline 32 & HD 73882 & 0.69 & 21.11 & & & & $21.11 \pm 0.15$ (FM90) \\
\hline 33 & HD 74194 & 0.50 & 21.23 & & & & 21.23 (scaled) \\
\hline 34 & HD 74920 & 0.35 & 21.15 & & $21.15 \pm 0.09$ & & \\
\hline 35 & HD 89137 & 0.23 & 21.03 & & & $21.03_{-0.02}^{+0.07}$ & \\
\hline 36 & HD 90087 & 0.28 & 21.19 & & $21.15 \pm 0.06$ & $21.19_{-0.09}^{+0.05}$ & \\
\hline 37 & HD 91597 & 0.30 & 21.40 & $21.34 \pm 0.10$ & $21.40 \pm 0.06$ & & \\
\hline 38 & HD 91651 & 0.28 & 21.15 & & $21.15 \pm 0.06$ & & \\
\hline 39 & HD 91824 & 0.25 & 21.12 & & & $21.12_{-0.04}^{+0.04}$ & $21.15 \pm 0.15($ FM90 $)$ \\
\hline 40 & HD 92554 & 0.39 & 21.34 & & $21.28 \pm 0.10$ & $21.34_{-0.11}^{+0.09}$ & \\
\hline 41 & HD 93028 & 0.24 & 20.95 & & & & $20.95 \pm 0.15$ (FM90) \\
\hline 42 & HD 93129A & 0.57 & 21.47 & & & $21.47_{-0.04}^{+0.07}$ & \\
\hline 43 & HD $93146 \mathrm{~A}$ & 0.35 & 21.18 & & $21.18 \pm 0.09$ & & \\
\hline 44 & HD 93204 & 0.41 & 21.41 & $21.40 \pm 0.10$ & $21.41 \pm 0.10$ & & \\
\hline 45 & HD 93205 & 0.40 & 21.36 & $21.34 \pm 0.05$ & $21.33 \pm 0.10$ & $21.36_{-0.05}^{+0.05}$ & \\
\hline 46 & HD 93206 & 0.39 & 21.34 & & $21.34 \pm 0.11$ & & \\
\hline 47 & HD 93222 & 0.37 & 21.47 & & & $21.47_{-0.04}^{+0.03}$ & $21.54 \pm 0.15(\mathrm{FM} 90)$ \\
\hline 48 & HD 93250 & 0.49 & 21.39 & $21.26 \pm 0.10$ & $21.39 \pm 0.15$ & & \\
\hline 49 & HD 93843 & 0.28 & 21.30 & $21.30 \pm 0.05$ & $21.33 \pm 0.08$ & $21.30_{-0.04}^{+0.05}$ & \\
\hline 50 & HD 96670 & 0.46 & 21.28 & & & & 21.28 (scaled) \\
\hline 51 & HD 96715 & 0.42 & 21.20 & $21.15 \pm 0.10$ & $21.20 \pm 0.16$ & & \\
\hline 52 & HD 96917 & 0.39 & 21.23 & & $21.23 \pm 0.11$ & & \\
\hline 53 & HD 97471 & 0.30 & 21.20 & & & & 21.20 (scaled) \\
\hline 54 & HD 97913 & 0.32 & 21.20 & & & & 21.20 (scaled) \\
\hline
\end{tabular}


Table 2-Continued

\begin{tabular}{|c|c|c|c|c|c|c|c|}
\hline ID & $\begin{array}{l}\text { Target } \\
\text { (Star) }\end{array}$ & $\begin{array}{l}E_{B-V}{ }^{\mathrm{b}} \\
(\mathrm{mag})\end{array}$ & $\begin{array}{c}\log N_{\mathrm{HI}}{ }^{\mathrm{c}} \\
\text { (Adopted) }\end{array}$ & $\begin{array}{l}\log N_{\mathrm{HI}} \\
(\mathrm{SVS} 85)\end{array}$ & $\begin{array}{c}\log N_{\mathrm{HI}} \\
(\mathrm{DS} 94)\end{array}$ & $\begin{array}{c}\log N_{\mathrm{HI}} \\
(\mathrm{J} 19)\end{array}$ & $\begin{array}{l}\log N_{\mathrm{HI}}{ }^{c} \\
\text { (Other) }\end{array}$ \\
\hline 55 & HD 99857 & 0.35 & 21.27 & & $21.31 \pm 0.12$ & $21.27_{-0.03}^{+0.07}$ & \\
\hline 56 & HD 99890 & 0.24 & 21.12 & & $20.93 \pm 0.13$ & $21.12_{-0.07}^{-0.03}$ & \\
\hline 57 & HD 100199 & 0.30 & 21.18 & & & $21.18_{-0.13}^{+0.07}$ & \\
\hline 58 & HD 100213 & 0.34 & 21.18 & & $21.18 \pm 0.07$ & & \\
\hline 59 & HD 100276 & 0.28 & 21.19 & & $21.19 \pm 0.09$ & & \\
\hline 60 & HD 101131 & 0.31 & 21.15 & & & & 21.15 (scaled) \\
\hline 61 & HD 101190 & 0.36 & 21.24 & $21.04 \pm 0.10$ & $21.15 \pm 0.11$ & $21.24_{-0.06}^{+0.03}$ & \\
\hline 62 & HD 101205 & 0.38 & 21.20 & & $21.20 \pm 0.07$ & & \\
\hline 63 & HD 101298 & 0.38 & 21.26 & & $21.26 \pm 0.11$ & & \\
\hline 64 & HD 101413 & 0.36 & 21.23 & & $21.23 \pm 0.13$ & & \\
\hline 65 & HD 101436 & 0.38 & 21.23 & & $21.23 \pm 0.08$ & & \\
\hline 66 & HD 103779 & 0.23 & 21.17 & & $21.16 \pm 0.10$ & $21.17_{-0.05}^{+0.05}$ & \\
\hline 67 & HD 104705 & 0.26 & 21.15 & & $21.11 \pm 0.07$ & $21.15_{-0.05}^{+0.05}$ & \\
\hline 68 & HD 115071 & 0.51 & 21.39 & & $21.38 \pm 0.10$ & $21.39_{-0.09}^{-0.05}$ & \\
\hline 69 & HD 116781 & 0.43 & 21.21 & & & $21.21_{-0.05}^{+0.05}$ & \\
\hline 70 & HD 116852 & 0.22 & 20.96 & $20.95 \pm 0.10$ & $20.96 \pm 0.08$ & $20.96_{-0.04}^{+0.05}$ & \\
\hline 71 & HD 118571 & 0.26 & 20.98 & & & & 20.98 (scaled) \\
\hline 72 & HD $124314 \mathrm{~A}$ & 0.53 & 21.41 & & $21.34 \pm 0.10$ & $21.41_{-0.04}^{+0.06}$ & \\
\hline 73 & HD 124979 & 0.41 & 21.27 & & $21.30 \pm 0.11$ & $21.27_{-0.09}^{+0.04}$ & \\
\hline 74 & HD 148422 & 0.35 & 21.24 & & $21.15 \pm 0.12$ & $21.24_{-0.06}^{+0.09}$ & \\
\hline 75 & HD 152218 & 0.48 & 21.34 & $21.34 \pm 0.10$ & $21.34 \pm 0.12$ & & \\
\hline 76 & HD 152233 & 0.45 & 21.29 & $21.35 \pm 0.10$ & $21.29 \pm 0.10$ & & \\
\hline 77 & HD 152248 & 0.47 & 21.37 & & & & 21.37 (scaled) \\
\hline 78 & HD 152314 & 0.50 & 21.35 & & & & 21.35 (scaled) \\
\hline 79 & HD 152623 & 0.40 & 21.28 & $21.11 \pm 0.05$ & $21.28 \pm 0.10$ & & \\
\hline 80 & HD 152723 & 0.47 & 21.43 & & $21.43 \pm 0.13$ & & \\
\hline 81 & HD 153426 & 0.45 & 21.34 & & $21.34 \pm 0.13$ & & \\
\hline 82 & HD 154368 & 0.81 & 21.00 & & & & $21.00 \pm 0.05(\mathrm{Sno} 96)$ \\
\hline 83 & HD 156292 & 0.56 & 21.29 & & & & 21.29 (scaled) \\
\hline 84 & HD 157857 & 0.49 & 21.30 & & $21.30 \pm 0.09$ & & \\
\hline 85 & HD 158661 & 0.38 & 21.28 & & & & 21.28 (scaled) \\
\hline 86 & HD 161807 & 0.23 & 21.08 & & & & 21.08 (scaled) \\
\hline 87 & HD 163758 & 0.35 & 21.23 & $21.20 \pm 0.10$ & $21.23 \pm 0.18$ & & \\
\hline 88 & HD 163892 & 0.46 & 21.32 & & $21.32 \pm 0.10$ & & \\
\hline 89 & HD 164816 & 0.31 & 21.18 & & $21.18 \pm 0.13$ & & \\
\hline 90 & HD 165052 & 0.42 & 21.36 & & $21.36 \pm 0.10$ & & \\
\hline 91 & HD 165246 & 0.40 & 21.41 & & & $21.41_{-0.04}^{+0.03}$ & \\
\hline 92 & HD 166546 & 0.34 & 21.19 & & & & 21.19 (scaled) \\
\hline 93 & HD 166716 & 0.38 & 21.27 & & & & 21.27 (scaled) \\
\hline 94 & HD 167402 & 0.23 & 21.13 & & $20.99 \pm 0.13$ & $21.13_{-0.04}^{+0.05}$ & \\
\hline 95 & HD 167659 & 0.53 & 21.30 & & $21.30 \pm 0.12$ & & \\
\hline 96 & HD 167771 & 0.44 & 21.08 & & $21.08 \pm 0.12$ & & $21.10 \pm 0.15($ FM90 $)$ \\
\hline 97 & HD 167971 & 1.08 & 21.60 & & & & $21.60 \pm 0.30($ Rat02 $)$ \\
\hline 98 & HD 168076 & 0.75 & 21.65 & & $21.65 \pm 0.23$ & & \\
\hline 99 & HD 168941 & 0.37 & 21.18 & & $21.11 \pm 0.09$ & $21.18_{-0.07}^{+0.05}$ & \\
\hline 100 & HD 172140 & 0.22 & 21.11 & & $21.11 \pm 0.08$ & & \\
\hline 101 & HD 175754 & 0.23 & 21.04 & $21.08 \pm 0.05$ & $21.04 \pm 0.10$ & & \\
\hline 102 & HD 175876 & 0.21 & 21.04 & & $21.04 \pm 0.11$ & & \\
\hline 103 & HD 177989 & 0.25 & 20.99 & & $20.95 \pm 0.09$ & $20.99_{-0.06}^{+0.05}$ & \\
\hline 104 & HD 178487 & 0.40 & 21.22 & & $21.15 \pm 0.10$ & $21.22_{-0.10}^{+0.04}$ & \\
\hline 105 & HD 179406 & 0.33 & 21.23 & & & & $21.23 \pm 0.30(\operatorname{Han} 92)$ \\
\hline 106 & HD 179407 & 0.33 & 21.20 & & $21.11 \pm 0.11$ & $21.20_{-0.10}^{+0.06}$ & \\
\hline 107 & HD 185418 & 0.50 & 21.19 & & & $21.19_{-0.04}^{-0.10}+0.05$ & $21.11 \pm 0.15$ (FM90) \\
\hline 108 & HD 187459 & 0.44 & 21.31 & & & & 21.31 (scaled) \\
\hline
\end{tabular}


Table 2 - Continued

\begin{tabular}{|c|c|c|c|c|c|c|c|}
\hline ID & $\begin{array}{l}\text { Target } \\
\text { (Star) }\end{array}$ & $\begin{array}{l}E_{B-V}{ }^{\mathrm{b}} \\
(\mathrm{mag})\end{array}$ & $\begin{array}{c}\log N_{\mathrm{HI}}{ }^{\mathrm{c}} \\
\text { (Adopted) }\end{array}$ & $\begin{array}{l}\log N_{\mathrm{HI}} \\
(\mathrm{SVS} 85)\end{array}$ & $\begin{array}{c}\log N_{\mathrm{HI}} \\
(\mathrm{DS} 94)\end{array}$ & $\begin{array}{c}\log N_{\mathrm{HI}} \\
(\mathrm{J} 19)\end{array}$ & $\begin{array}{l}\log N_{\mathrm{HI}}{ }^{\mathrm{c}} \\
\text { (Other) }\end{array}$ \\
\hline 109 & HD 190429A & 0.43 & 21.33 & & & & 21.33 (scaled) \\
\hline 110 & HD 190918 & 0.45 & 21.40 & & $21.40 \pm 0.10$ & & \\
\hline 111 & HD 191495 & 0.40 & 21.32 & & & & 21.32 (scaled) \\
\hline 112 & HD 191877 & 0.21 & 21.03 & & $20.90 \pm 0.10$ & $21.03_{-0.05}^{+0.05}$ & \\
\hline 113 & HD 192035 & 0.34 & 21.20 & & $21.09 \pm 0.19$ & $21.20_{-0.10}^{+0.04}$ & \\
\hline 114 & HD 192639 & 0.66 & 21.32 & & $21.32 \pm 0.12$ & & \\
\hline 115 & HD 195965 & 0.25 & 20.92 & $20.95 \pm 0.07$ & $20.90 \pm 0.09$ & $20.92_{-0.05}^{+0.05}$ & \\
\hline 116 & HD 199579 & 0.37 & 21.04 & $21.04 \pm 0.10$ & $21.04 \pm 0.11$ & & $21.08 \pm 0.15($ FM90) \\
\hline 117 & HD 201345 & 0.15 & 21.00 & & $20.88 \pm 0.10$ & $21.00_{-0.06}^{+0.05}$ & \\
\hline 118 & HD 201638 & 0.11 & 20.80 & & & & 20.80 (scaled) \\
\hline 119 & HD $203374 \mathrm{~A}$ & 0.53 & 21.20 & & & $21.20_{-0.04}^{+0.05}$ & \\
\hline 120 & HD 206267 & 0.53 & 21.22 & & & $21.22_{-0.04}^{+0.06}$ & $21.30 \pm 0.15($ Rat02 $)$ \\
\hline 121 & HD 206773 & 0.51 & 21.09 & & & $21.09_{-0.03}^{+0.07}$ & \\
\hline 122 & HD 207198 & 0.60 & 21.28 & $21.34 \pm 0.15$ & $21.34 \pm 0.17$ & $21.28_{-0.09}^{+0.07}$ & \\
\hline 123 & HD 207308 & 0.53 & 21.20 & & & $21.20_{-0.05}^{+0.06}$ & \\
\hline 124 & HD 208440 & 0.28 & 21.24 & & $21.23 \pm 0.07$ & $21.24_{-0.04}^{+0.06}$ & \\
\hline 125 & HD 209339 & 0.30 & 21.20 & & & $21.20_{-0.04}^{+0.04}$ & \\
\hline 126 & HD 210809 & 0.34 & 21.31 & & $21.26 \pm 0.07$ & $21.31_{-0.05}^{+0.06}$ & \\
\hline 127 & HD 210839 & 0.57 & 21.24 & $21.20 \pm 0.10$ & $21.15 \pm 0.12$ & $21.24_{-0.05}^{+0.05}$ & \\
\hline 128 & HD 216044 & 0.38 & 21.28 & & & & 21.28 (scaled) \\
\hline 129 & HD 216532 & 0.85 & 21.38 & & & & 21.38 (scaled) \\
\hline 130 & HD 216898 & 0.84 & 21.44 & & & & 21.44 (scaled) \\
\hline 131 & HD 217035 & 0.74 & 21.46 & & $21.46 \pm 0.12$ & & \\
\hline 132 & HD 217312 & 0.67 & 21.48 & & $21.48 \pm 0.09$ & & \\
\hline 133 & HD 218915 & 0.30 & 21.20 & $21.20 \pm 0.10$ & $21.11 \pm 0.13$ & $21.20_{-0.06}^{+0.07}$ & \\
\hline 134 & HD 224151 & 0.44 & 21.35 & & $21.32 \pm 0.10$ & $21.35_{-0.08}^{+0.05}$ & \\
\hline 135 & HD 224257 & 0.24 & 21.08 & & & & 21.08 (scaled) \\
\hline 136 & HD 224868 & 0.34 & 21.16 & & & & 21.16 (scaled) \\
\hline 137 & HD 303308 & 0.46 & 21.41 & $21.40 \pm 0.10$ & $21.45 \pm 0.09$ & $21.41_{-0.08}^{+0.03}$ & \\
\hline 138 & HD 308813 & 0.34 & 21.20 & & $21.15 \pm 0.10$ & $21.20_{-0.04}^{+0.06}$ & \\
\hline 139 & HD 332407 & 0.41 & 21.24 & & $21.24 \pm 0.14$ & & \\
\hline
\end{tabular}

${ }^{\text {a }}$ The adopted column densities $N_{\mathrm{HI}}\left(\right.$ in $\mathrm{cm}^{-2}$ ) in column 4 (SD20) are compared to three previous Ly $\alpha$-fitting surveys in columns 5-7: SVS85 (Shull \& Van Steenberg 1985); DS94 (Diplas \& Savage 1994); and J19 (Jenkins 2019). Column 8 lists individual measurements from: FM90 (Fitzpatrick \& Massa 1990); Rat02 (Rachford et al. 2002); Han92 (Hanson et al. 1992); Sno96 (Snow et al. 1996); and scaled estimates of $N_{\text {Hi }}$ from $E(B-V)$. Footnote (c) to Table 3 provides further details.

${ }^{\mathrm{b}}$ Updated values of color excess (Shull \& Danforth 2019) were derived using digital photometry, visual extinction, and new SpTs from the Galactic O-star Spectroscopic Survey (Maíz Apellániz et al. 2004; Sota et al. 2011, 2014).

${ }^{\mathrm{c}}$ The adopted source for $N_{\mathrm{HI}}$ was selected in priority order of: J19 (57 stars), DS94 (51 stars), FM90 (2 stars), and three other sources ( 3 stars). For 26 stars that lack fits to Ly $\alpha$ profiles, we estimated $N_{\mathrm{HI}}$ by scaling with color excess $E(B-V)$; see Section 2.1 . 
Table 3. Column Densities ${ }^{\mathrm{a}}$ and Doppler Parameters ${ }^{\mathrm{a}}$

\begin{tabular}{|c|c|c|c|c|c|c|c|c|c|}
\hline ID & Target & $\log \mathrm{N}(0)$ & $\log N(1)$ & $\log \mathrm{N}(2)$ & $\log N(3)$ & $\log N(4)$ & $\log N(5)$ & $\log \mathrm{N}(6)$ & $b\left(\mathrm{~km} \mathrm{~s}^{-1}\right)$ \\
\hline 1 & BD $35^{\circ} 4258$ & $19.03_{-0.10}^{+0.10}$ & $19.39_{-0.10}^{+0.10}$ & $17.72_{-0.30}^{+0.21}$ & $17.78_{-0.32}^{+0.23}$ & $15.68_{-0.14}^{+0.20}$ & $15.36_{-0.12}^{+0.17}$ & $14.14_{-0.18}^{+0.18}$ & $8.4_{-1.0}^{+0.9}$ \\
\hline 2 & $\mathrm{BD} 53^{\circ} 2820$ & $19.59_{-0.10}^{+0.10}$ & $19.85_{-0.10}^{+0.10}$ & $16.07_{-0.40}^{+0.40}$ & $15.85_{-0.4}^{+0.4}$ & $15.25_{-0.02}^{+0.04}$ & $14.95_{-0.02}^{+0.03}$ & $<14.15$ & $20_{-1.0}^{+1.0}$ \\
\hline 3 & $\mathrm{CPD}-59^{\circ} 2600$ & $19.84_{-0.10}^{+0.10}$ & $19.88_{-0.10}^{+0.10}$ & $16.66_{-0.32}^{+0.40}$ & $16.39_{-0.20}^{+0.46}$ & $15.06_{-0.07}^{+0.02}$ & $14.89_{-0.06}^{+0.08}$ & $14.04_{-0.10}^{+0.10}$ & $8.4_{-1.0}^{+0.9}$ \\
\hline 4 & $\mathrm{CPD}-59^{\circ} 2603$ & $19.77_{-0.05}^{+0.05}$ & $19.87_{-0.10}^{+0.10}$ & $18.23_{-0.50}^{+0.52}$ & $15.65_{-0.73}^{+0.20}$ & $14.88_{-0.05}^{+0.05}$ & $14.76_{-0.06}^{+0.06}$ & $13.94_{-0.18}^{+0.10}$ & $11.3_{-1.9}^{+0.9}$ \\
\hline 5 & $\mathrm{CPD}-69^{\circ} 1743$ & $19.49_{-0.10}^{+0.10}$ & $19.71_{-0.10}^{+0.10}$ & $15.78_{-0.08}^{+0.50}$ & $15.51_{-0.08}^{+0.10}$ & $14.68_{-0.09}^{+0.09}$ & $14.39_{-0.04}^{+0.06}$ & $<13.85$ & $13.3_{-1.0}^{+0.9}$ \\
\hline 6 & $\mathrm{CPD}-72^{\circ} 1184$ & $19.59_{-0.10}^{+0.10}$ & $19.90_{-0.10}^{+0.10}$ & $17.24_{-0.50}^{+0.70}$ & $16.04_{-0.20}^{+1.48}$ & $14.56_{-0.03}^{+1.09}$ & $<14.30$ & $<14.10$ & $\ldots{ }^{-1.0}$ \\
\hline 7 & HD 3827 & $16.84_{-0.05}^{+0.05}$ & $17.33_{-0.05}^{+0.05}$ & $16.15_{-0.13}^{+0.20}$ & $15.82_{-0.10}^{+0.14}$ & $14.55_{-0.06}^{+0.06}$ & $14.16_{-0.09}^{+0.09}$ & $<13.85$ & $12.8_{-1.0}^{+0.9}$ \\
\hline 8 & HD $5005 \mathrm{~A}$ & $19.87_{-0.05}^{+0.05}$ & $19.96_{-0.05}^{+0.05}$ & $18.16_{-0.50}^{+0.50}$ & $17.14_{-0.28}^{+0.27}$ & $15.46_{-0.09}^{+0.04}$ & $15.00_{-0.07}^{+0.07}$ & $14.08_{-010}^{+0.10}$ & $15.0_{-1.6}^{+3.6}$ \\
\hline 9 & HD 12323 & $19.92_{-0.10}^{+0.10}$ & $20.08_{-0.10}^{+0.10}$ & $18.01_{-0.25}^{+0.18}$ & $17.35_{-0.45}^{+0.68}$ & $15.45_{-0.15}^{+0.23}$ & $14.81_{-0.06}^{+0.08}$ & $<14.00$ & $7.4_{-10}^{+1.0}$ \\
\hline \multirow[t]{3}{*}{10} & HD $13268^{\mathrm{b}}$ & $20.00_{-0.10}^{+0.10}$ & $20.29_{-0.10}^{+0.10}$ & $18.08_{-0.50}^{+0.50}$ & $17.23_{-0.50}^{+0.50}$ & $15.53_{-0.03}^{+0.03}$ & $15.18_{-0.03}^{+0.03}$ & $<14.00$ & $\ldots$ \\
\hline & (blue) & $\ldots$ & $\ldots$ & $\ldots$ & $\ldots$ & $14.75_{-0.04}^{+0.04}$ & $14.52_{-0.04}^{+0.04}$ & $\ldots$ & $15.6_{+10}^{+4.4}$ \\
\hline & (red) & $\ldots$ & $\cdots$ & 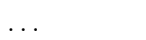 & 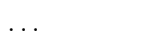 & $15.45_{-0.04}^{+0.04}$ & $15.07_{-0.04}^{+0.04}$ & $\ldots$ & $13.2_{-1.0}^{+0.9}$ \\
\hline \multirow[t]{3}{*}{11} & HD $13745^{\mathrm{b}}$ & $20.30_{-0.10}^{+0.10}$ & $20.27_{-0.10}^{+0.10}$ & $18.74_{-0.50}^{+0.50}$ & $17.38_{-0.33}^{+0.35}$ & $15.74_{-0.06}^{+1.77}$ & $\begin{array}{l}15.09_{-0.03}^{+0.09} \\
+0.03\end{array}$ & $<14.00$ & $\ldots$ \\
\hline & (blue) & $\ldots$ & $\ldots$ & $\ldots{ }^{-0.00}$ & $17.27_{-0.46}^{+0.43}$ & $15.07_{-0.16}^{+1.80}$ & $14.29_{-0.04}^{+0.03}$ & $\ldots$ & $3.9_{-2.9}^{+1.0}$ \\
\hline & (red) & $\ldots$ & $\ldots$ & $\ldots$ & $16.74_{-0.28}^{+0.38}$ & $15.63_{-0.06}^{+0.08}$ & $15.02_{-0.04}^{+0.04}$ & ... & $9.6_{-1.0}^{+\overline{0} .9}$ \\
\hline \multirow[t]{3}{*}{12} & HD $14434^{\mathrm{b}}$ & $20.04_{-0.10}^{+0.10}$ & $20.36_{-0.10}^{+0.10}$ & $18.94_{-0.50}^{+0.50}$ & $17.06_{-0.20}^{+0.27}$ & $15.59_{-0.03}^{+0.05}$ & $15.12_{-0.03}^{+0.03}$ & $<14.00$ & $\ldots$ \\
\hline & (blue) & $\ldots$ & $\cdots$ & $\ldots$ & $16.93_{-0.27}^{+0.35}$ & $15.17_{-0.04}^{+0.04}$ & $14.88_{-0.04}^{+0.04}$ & $\ldots$ & $\ldots$ \\
\hline & (red) & $\ldots$ & $\ldots$ & $\ldots$ & $16.50_{-0.24}^{+0.37}$ & $15.38_{-0.05}^{+0.08}$ & $14.74_{-0.04}^{+0.04}$ & $\ldots$ & $\ldots$ \\
\hline 13 & HD 15137 & $19.77_{-0.05}^{+0.05}$ & $20.10_{-0.05}^{+0.05}$ & $17.55_{-0.35}^{+0.33}$ & $16.74_{-0.30}^{+0.40}$ & $15.44_{-0.08}^{+0.11}$ & $14.58_{-0.04}^{+0.05}$ & $\ldots$ & $9.5_{-1.0}^{+0.9}$ \\
\hline 14 & HD $15558 \mathrm{~A}$ & $20.50_{-0.15}^{+0.15}$ & $20.66_{-0.10}^{+0.10}$ & $19.04_{-0.50}^{+0.50}$ & $17.39_{-0.26}^{+0.29}$ & $15.70_{-0.07}^{+0.09}$ & $15.36_{-0.05}^{+0.06}$ & $<14.60$ & $12.6_{-1.0}^{+0.9}$ \\
\hline \multirow[t]{3}{*}{15} & HD $15642^{\mathrm{b}}$ & $19.61_{-0.10}^{+0.10}$ & $20.10_{-0.10}^{+0.10}$ & $19.64_{-0.50}^{+0.50}$ & $18.14_{-0.50}^{+0.50}$ & $15.58_{-0.12}^{+0.21}$ & $14.88_{-0.04}^{+0.04}$ & $<14.00$ & $\cdots$ \\
\hline & (blue) & $\ldots$ & $\cdots$ & $\ldots$ & $\ldots$ & $14.46_{-0.04}^{+0.04}$ & $13.88_{-0.04}^{+0.04}$ & $\cdots$ & $\cdots$ \\
\hline & (red) & $\ldots$ & $\ldots$ & $\ldots$ & $\ldots$ & $15.58_{-0.12}^{+0.21}$ & $14.88_{-0.04}^{+0.04}$ & $\ldots$ & $\ldots$ \\
\hline 16 & HD 34656 & $19.80_{-0.05}^{+0.05}$ & $19.97_{-0.05}^{+0.05}$ & $17.16_{-0.50}^{+0.50}$ & $16.86_{-0.28}^{+0.37}$ & $15.28_{-0.06}^{+0.08}$ & $14.71_{-0.04}^{+0.04}$ & $<13.95$ & $10.8_{-1.0}^{+0.9}$ \\
\hline 17 & HD 39680 & $19.16_{-0.05}^{+0.05}$ & $19.29_{-0.05}^{+0.05}$ & $16.82_{-0.47}^{+0.54}$ & $16.37_{-0.24}^{+0.48}$ & $14.83_{-0.05}^{+0.10}$ & $14.20_{-0.04}^{+0.04}$ & $<14.15$ & $6.3_{-1.0}^{+1.0}$ \\
\hline 18 & HD 41161 & $19.62_{-0.15}^{+0.15}$ & $19.80_{-0.15}^{+0.15}$ & $17.58_{-0.50}^{+0.50}$ & $17.01_{-0.36}^{+0.40}$ & $14.97_{-0.06}^{+0.09}$ & $14.28_{-0.05}^{+0.04}$ & $<14.15$ & $7.7_{-1.0}^{+0.9}$ \\
\hline 19 & HD 42088 & $20.18_{-0.05}^{+0.05}$ & $20.23_{-0.10}^{+0.10}$ & $17.77_{-0.25}^{+0.25}$ & $17.22_{-0.39}^{+0.39}$ & $15.34_{-0.12}^{+0.12}$ & $14.90_{-0.06}^{+0.05}$ & $<13.70$ & $7.5_{-1.9}^{+0.9}$ \\
\hline 20 & HD 45314 & $20.23_{-0.05}^{+0.05}$ & $20.35_{-0.05}^{+0.05}$ & $18.05_{-0.18}^{+0.11}$ & $17.28_{-0.46}^{+0.31}$ & $15.09_{-0.08}^{+0.10}$ & $14.30_{-0.04}^{+0.04}$ & $<14.08$ & $6.2_{-1.0}^{+1.0}$ \\
\hline 21 & HD 46150 & $20.23_{-0.05}^{+0.05}$ & $20.42_{-0.05}^{+0.05}$ & $16.91_{-028}^{+0.39}$ & $16.36_{-0.30}^{+0.30}$ & $15.30_{-0.07}^{+0.07}$ & $14.87_{-0.05}^{+0.05}$ & $13.76_{-015}^{+0.15}$ & $10.8_{-10}^{+0.9}$ \\
\hline 22 & HD 47360 & $20.02_{-0.10}^{+0.10}$ & $20.13_{-0.10}^{+0.10}$ & $17.71_{-0.32}^{+0.21}$ & $16.55_{-044}^{+0.54}$ & $15.31_{-0.16}^{+0.16}$ & $14.78_{-0.08}^{+0.08}$ & $<14.00$ & $6.8_{-1.0}^{+1.0}$ \\
\hline 23 & HD 47417 & $20.15_{-0.05}^{+0.05}$ & $20.27_{-0.05}^{+0.05}$ & $17.74_{-022}^{+0.22}$ & $17.20_{-0.32}^{+0.35}$ & $15.29_{-0.06}^{+0.06}$ & $14.73_{-0.05}^{+0.05}$ & $<14.08$ & $9.0_{-10}^{+0.9}$ \\
\hline 24 & HD 60369 & $20.25_{-0.05}^{+0.05}$ & $20.10_{-0.05}^{+0.05}$ & $17.96_{-015}^{+0.15}$ & $17.66_{-029}^{+0.23}$ & $15.50_{-022}^{+0.57}$ & $14.90_{-0.05}^{+0.10}$ & $<13.85$ & $5.3_{-11}^{+0.9}$ \\
\hline \multirow[t]{3}{*}{25} & HD $61347^{\mathrm{b}}$ & $20.27_{-0.10}^{+0.10}$ & $20.31_{-0.10}^{+0.10}$ & $18.74_{-0.50}^{+0.50}$ & $17.41_{-0.40}^{+0.62}$ & $15.48_{-0.06}^{+0.50}$ & $14.88_{-0.03}^{+0.75}$ & $<14.20$ & $\cdots$ \\
\hline & (blue) & $\ldots$ & $\cdots$ & .. & $15.53_{-0.24}^{+1.80}$ & $14.96_{-0.09}^{+0.40}$ & $14.25_{-0.04}^{+1.0}$ & .. & $\ldots$ \\
\hline & (red) & $\ldots$ & $\ldots$ & $\ldots$ & $17.41_{-0.40}^{+0.62}$ & $15.48_{-0.06}^{+0.50}$ & $14.88_{-0.04}^{+0.75}$ & $\ldots$ & $\ldots$ \\
\hline 26 & HD 62866 & $19.80_{-0.10}^{+0.10}$ & $20.04_{-0.10}^{+0.10}$ & $16.65_{-0.40}^{+0.40}$ & $16.27_{-0.17}^{+0.13}$ & $15.36_{-0.07}^{+0.05}$ & $14.95_{-0}^{+0}$ & $<14.30$ & $17.7_{-1.0}^{+2.2}$ \\
\hline 27 & HD 63005 & $19.68_{-0.10}^{+0.10}$ & $19.97_{-0.10}^{+0.10}$ & $17.73_{-0.25}^{+0.15}$ & $17.04_{-0.48}^{+0.36}$ & $15.22_{-0.11}^{+0.17}$ & $14.59_{-0.05}^{+0.06}$ & $<14.01$ & $5.4_{-0.8}^{+0.9}$ \\
\hline 28 & HD 64568 & $20.35_{-0.10}^{+0.10}$ & $20.10_{-0.10}^{+0.10}$ & $16.67_{-0.40}^{+0.40}$ & $16.51_{-0.21}^{+0.15}$ & $15.34_{-0 .}^{+0.6}$ & $14.96_{-0.04}^{+0.04}$ & $<14.10$ & $15.9_{-1.0}^{+2.7}$ \\
\hline 29 & HD 66695 & $19.82_{-0.10}^{+0.10}$ & $19.79_{-0.10}^{+0.10}$ & $18.21_{-0.11}^{+0.11}$ & $17.96_{-0.20}^{+0.16}$ & $16.02_{-0.2}^{+0.4}$ & $15.82_{-0.29}^{+0.48}$ & $<14.00$ & $6.8_{-1.0}^{+1.0}$ \\
\hline 30 & HD 66788 & $19.33_{-0.05}^{+0.05}$ & $19.42_{-0.05}^{+0.05}$ & $18.40_{-0.09}^{+0.06}$ & $18.24_{-0.14}^{+0.09}$ & $15.46_{-0.13}^{+0.20}$ & $14.65_{-0.04}^{+0.04}$ & $<14.18$ & $6.4_{-0.9}^{+1.0}$ \\
\hline 31 & HD 69106 & $19.41_{-0.10}^{+0.10}$ & $19.44_{-0.10}^{+0.10}$ & $17.77_{-0.50}^{+0.50}$ & $17.61_{-0.33}^{+0.28}$ & $15.38_{-0.1}^{+0.1}$ & $14.46_{-0.04}^{+0.04}$ & $<14.11$ & $9.6_{-1.0}^{+0.9}$ \\
\hline 32 & HD 73882 & $21.00_{-0.15}^{+0.15}$ & $20.55_{-0.15}^{+0.15}$ & $16.61_{-0.40}^{+0.40}$ & $16.04_{-0.0}^{+0.0}$ & $15.13_{-0.02}^{+0.02}$ & $15.28_{-0.20}^{+0.20}$ & $<14.40$ & $18.6_{-1.0}^{+1.3}$ \\
\hline 33 & HD 74194 & $20.14_{-0.10}^{+0.10}$ & $20.33_{-0.10}^{+0.10}$ & $18.05_{-0.15}^{+0.12}$ & $17.25_{-0.47}^{+0.40}$ & $15.45_{-0.1}^{+0.3}$ & $14.93_{-0.05}^{+0.07}$ & $<14.00$ & $5.7_{-1.0}^{+1.0}$ \\
\hline 34 & HD 74920 & $19.77_{-0.05}^{+0.05}$ & $20.09_{-0.05}^{+0.05}$ & $16.42_{-0.24}^{+0.37}$ & $16.06_{-0.16}^{+0.26}$ & $15.01_{-0}^{+0}$. & $14.60_{-0.09}^{+0.09}$ & $13.85_{-020}^{+0.20}$ & $8.9_{-10}^{+0.9}$ \\
\hline 35 & HD 89137 & $19.53_{-0.05}^{+0.05}$ & $19.81_{-0.05}^{+0.05}$ & $16.12_{-0.15}^{+0.25}$ & $15.70_{-0.10}^{+0.15}$ & $14.70_{-0.04}^{+0.04}$ & $14.28_{-0.12}^{+0.12}$ & $<13.85$ & $9.6_{-1.0}^{+0.9}$ \\
\hline 36 & HD 90087 & $19.53_{-0.05}^{+0.05}$ & $19.68_{-0.05}^{+0.05}$ & $17.57_{-0.37}^{+0.23}$ & $16.87_{-0.39}^{+0.41}$ & $15.36_{-0.09}^{+0.12}$ & $14.79_{-0.04}^{+0.05}$ & $13.63_{-0.04}^{+0.04}$ & $7.3_{-0.9}^{+1.0}$ \\
\hline 37 & HD 91597 & $19.45_{-0.10}^{+0.10}$ & $19.33_{-0.10}^{+0.10}$ & $16.20_{-0.17}^{+0.27}$ & $16.03_{-0.15}^{+0.23}$ & $15.11_{-0.05}^{+0.05}$ & $14.83_{-0.04}^{+0.04}$ & $<14.00$ & $10.9_{-1.0}^{+0.9}$ \\
\hline 38 & HD 91651 & $18.74_{-0.05}^{+0.05}$ & $18.79_{-0.05}^{+0.05}$ & $16.35_{-0.19}^{+0.28}$ & $16.53_{-0.24}^{+0.33}$ & $15.37_{-0.06}^{+0.08}$ & $15.11_{-0.05}^{+0.06}$ & $14.06_{-0.08}^{+0.08}$ & $10.9_{-1.0}^{+0.9}$ \\
\hline 39 & HD 91824 & $19.61_{-0.05}^{+0.05}$ & $19.43_{-0.05}^{+0.05}$ & $17.72_{-0.50}^{+0.50}$ & $17.17_{-0.42}^{+0.38}$ & $15.24_{-0.07}^{+0.09}$ & $14.64_{-0}^{+0 .}$ & $<14.00$ & $7.4_{-1.0}^{+1.0}$ \\
\hline 40 & HD 92554 & $18.88_{-0.10}^{+0.10}$ & $17.98_{-0.10}^{+0.10}$ & $15.94_{-0.10}^{+0.10}$ & $15.91_{-0.10}^{+0.13}$ & $14.98_{-0.04}^{+0.04}$ & $14.86_{-0.04}^{+0.04}$ & $14.27_{-0.08}^{+0.08}$ & $13.4_{-1.0}^{+0.9}$ \\
\hline 41 & HD 93028 & $19.08_{-0.10}^{+0.10}$ & $19.37_{-0.10}^{+0.10}$ & $15.98_{-0.11}^{+0.20}$ & $15.73_{-0.11}^{+0.19}$ & $14.72_{-0.04}^{+0.04}$ & $14.22_{-0.09}^{+0.09}$ & $<14.00$ & $9.1_{-1.0}^{+0.9}$ \\
\hline 42 & HD 93129A & $19.68_{-0.10}^{+0.10}$ & $20.06_{-0.10}^{+0.10}$ & $17.47_{-0.39}^{+0.36}$ & $17.88_{-0.30}^{+0.24}$ & $15.72_{-0.16}^{+0.29}$ & $15.36_{-0.09}^{+0.14}$ & $14.05_{-0.08}^{+0.08}$ & $8.7_{-1.0}^{+0.9}$ \\
\hline 43 & HD 93146A & $19.38_{-0.10}^{+0.10}$ & $19.43_{-0.10}^{+0.10}$ & $15.97_{-0.16}^{+0.24}$ & $15.78_{-0.13}^{+0.21}$ & $14.79_{-0.0}^{+0.0}$ & $14.56_{-0.04}^{+0.04}$ & $14.14_{-0.15}^{+0.15}$ & $9.1_{-1.0}^{+0.9}$ \\
\hline 44 & HD 93204 & $19.37_{-}^{+c}$ & $19.62_{-}^{+c}$ & $16.11_{-}^{+c}$ & $15.88_{-}^{+}$ & $14.86_{-}^{+}$ & $14.51_{-}^{+}$ & $<13.85$ & $9.2_{-1.0}^{+0.9}$ \\
\hline 45 & HD $93205^{\mathrm{b}}$ & $19.33_{-0.05}^{+0.05}$ & $19.61_{-0.05}^{+0.05}$ & $16.47_{-0.28}^{+0.44}$ & $16.29_{-0.18}^{+0.37}$ & $14.92_{-0.04}^{+0.06}$ & $14.59_{-0.04}^{+0.06}$ & $<13.85$ & \\
\hline
\end{tabular}


Table 3 - Continued

\begin{tabular}{|c|c|c|c|c|c|c|c|c|c|}
\hline ID & Target & $\log \mathrm{N}(0)$ & $\log N(1)$ & $\log \mathrm{N}(2)$ & $\log N(3)$ & $\log N(4)$ & $\log N(5)$ & $\log N(6)$ & $b\left(\mathrm{~km} \mathrm{~s}^{-1}\right)$ \\
\hline & (blue) & $\ldots$ & $\cdots$ & $14.36_{-0.12}^{+0.55}$ & $14.93_{-0.36}^{+1.10}$ & $13.95_{-0.06}^{+0.23}$ & $13.96_{-0.05}^{+0.17}$ & $\cdots$ & $\cdot$ \\
\hline & (red) & $\ldots$ & $\ldots$ & $16.47_{-0.28}^{+0.44}$ & $16.27_{-0.18}^{+0.36}$ & $\begin{array}{r}14.87_{-0.04}^{+0.05}\end{array}$ & $14.48_{-0.04}^{+0.05}$ & $\ldots$ & $\ldots$ \\
\hline 46 & HD 93206 & $19.18_{-0.05}^{+0.05}$ & $19.25_{-0.05}^{+0.05}$ & $15.87_{-0.13}^{+0.24}$ & $15.78_{-0.13}^{+0.10}$ & $14.48_{-0.04}^{+0.04}$ & $14.10_{-0.04}^{+0.04}$ & $<13.85$ & $8.2_{-10}^{+0.9}$ \\
\hline 47 & HD 93222 & $19.49_{-0.05}^{+0.05}$ & $19.44_{-0.05}^{+0.05}$ & $15.80_{-0.06}^{+0.08}$ & $15.70_{-0.07}^{+0.09}$ & $14.71_{-0.04}^{+0.04}$ & $14.46_{-0.04}^{+0.04}$ & $<14.15$ & $12.9_{-1.0}^{+0.9}$ \\
\hline 48 & HD 93250 & $19.79_{-0.05}^{+0.05}$ & $20.10_{-0.05}^{+0.05}$ & $16.37_{-0.07}^{+0.41}$ & $16.68_{-0.07}^{+0.24}$ & $15.75_{-0.07}^{+0.19}$ & $15.89_{-0.12}^{+0.19}$ & $15.59_{-0.15}^{+0.15}$ & $15.4_{-1.0}^{+0.9}$ \\
\hline 49 & HD 93843 & $19.14_{-0.05}^{+0.05}$ & $19.43_{-0.05}^{+0.05}$ & $16.04_{-0.08}^{+0.010}$ & $15.86_{-0.05}^{+0.06}$ & $14.67_{-0.04}^{+0.04}$ & $14.40_{-0.04}^{+0.04}$ & $<13.78$ & $14.6_{-1.0}^{+0.9}$ \\
\hline 50 & HD 96670 & $20.33_{-0.10}^{+0.10}$ & $20.24_{-0.10}^{+0.05}$ & $18.56_{-0.50}^{+0.50}$ & $16.70_{-0.33}^{+0.29}$ & $\begin{array}{l}15.74_{-0.12}^{+0.18} \\
\end{array}$ & $15.00_{-0.06}^{+0.08}$ & $<14.00$ & $9.1_{-1.0}^{+0.9}$ \\
\hline 51 & HD 96715 & $20.45_{-0.10}^{+0.10}$ & $20.11_{-0.10}^{+0.10}$ & $18.53_{-0.50}^{+0.50}$ & $17.15_{-0.29}^{+0.29}$ & $15.66_{-0.07}^{+0.07}$ & $14.82_{-0.04}^{+0.04}$ & $<14.10$ & $10.1_{-0.9}^{+0.9}$ \\
\hline 52 & HD $96917^{\mathrm{c}}$ & $20.04_{-0.10}^{+0.10}$ & $19.83_{-0.10}^{+0.10}$ & $\ldots$ & $\ldots$ & $16.18_{-0.12}^{+0.12}$ & $15.43_{-0.15}^{+0.15}$ & $14.43_{-0.05}^{+0.05}$ & $12.0_{-10}^{+3.0}$ \\
\hline 53 & HD 97471 & $19.67_{-0.10}^{+0.10}$ & $19.52_{-0.10}^{+0.10}$ & $17.15_{-0.31}^{+0.36}$ & $16.74_{-0.27}^{+0.37}$ & $15.33_{-0.09}^{+0.11}$ & $14.77_{-0.04}^{+0.04}$ & $<13.95$ & $10.6_{-1.0}^{+0.9}$ \\
\hline 54 & HD 97913 & $19.76_{-0.10}^{+0.10}$ & $19.89_{-0.10}^{+0.10}$ & $16.96_{-0.30}^{+0.31}$ & $16.46_{-0.23}^{+0.33}$ & $15.32_{-0.06}^{+0.09}$ & $14.69_{-0.04}^{+0.04}$ & $13.90_{-0.10}^{+0.10}$ & $10.5_{-1.0}^{+0.9}$ \\
\hline 55 & HD 99857 & $19.90_{-0.10}^{+0.10}$ & $20.01_{-0.10}^{+0.10}$ & $18.19_{-0.11}^{+0.08}$ & $17.78_{-0.26}^{+0.15}$ & $15.05_{-0.07}^{+0.09}$ & $14.46_{-0.04}^{+0.04}$ & $<14.04$ & $5.7_{-0.8}^{+1.0}$ \\
\hline 56 & HD 99890 & $19.09_{-0.10}^{+0.10}$ & $19.23_{-0.10}^{+0.10}$ & $16.84_{-0.27}^{+0.40}$ & $16.66_{-0.25}^{+0.38}$ & $15.01_{-0.06}^{+0.08}$ & $14.56_{-0.04}^{+0.04}$ & $<13.85$ & $9.4_{-1}^{+0}$ \\
\hline 57 & HD 100199 & $19.76_{-0.10}^{+0.10}$ & $19.96_{-0.10}^{+0.10}$ & $17.66_{-0.34}^{+0.26}$ & $17.11_{-0.35}^{+0.39}$ & $15.35_{-0.09}^{+0.11}$ & $14.75_{-0.04}^{+0.05}$ & $<14.08$ & $8.4_{-1}^{+c}$ \\
\hline 58 & HD 100213 & $20.11_{-0.10}^{+0.10}$ & $20.13_{-0.10}^{+0.10}$ & $18.22_{-0.17}^{+0.34}$ & $17.61_{-0.41}^{+0.25}$ & $15.33_{-0.10}^{+0.09}$ & $14.65_{-0.05}^{+0.05}$ & $<13.98$ & $7.2_{-1}^{+1}$ \\
\hline 59 & HD 100276 & $19.49_{-0.10}^{+0.10}$ & $19.57_{-0.10}^{+0.10}$ & $16.44_{-0.15}^{+0.15}$ & $16.02_{-0.12}^{+0.41}$ & $15.07_{-0.04}^{+0.04}$ & $15.05_{-0.04}^{+0.04}$ & $<14.10$ & $17.0_{-1.0}^{+2.0}$ \\
\hline 60 & HD 101131 & $19.92_{-0.05}^{+0.05}$ & $20.02_{-0.05}^{+0.05}$ & $18.10_{-0.21}^{+0.08}$ & $17.70_{-0.38}^{+0.12}$ & $15.27_{-0.10}^{-0.04}$ & $14.59_{-0.06}^{-0.04}$ & $<14.18$ & $7.1_{-0}^{+1}$ \\
\hline 61 & HD 101190 & $20.21_{-0.05}^{+0.05}$ & $20.00_{-0.05}^{+0.05}$ & $18.33_{-0.07}^{+0.03}$ & $17.81_{-0.18}^{+0.08}$ & $15.16_{-0.07}^{+0.05}$ & $14.44_{-0.04}^{+0.00}$ & $<13.95$ & $4.9_{-0.4}^{+0.0}$ \\
\hline 62 & HD 101205 & $19.94_{-0.15}^{+0.05}$ & $19.96_{-0.15}^{+0.15}$ & $18.48_{-0.10}^{+0.07}$ & $17.98_{-0.23}^{+0.16}$ & $15.45_{-0.17}^{+0.33}$ & $14.60_{-0.05}^{+0.04}$ & $<14.18$ & $6.2_{-1.0}^{+0.0}$ \\
\hline 63 & HD 101298 & $20.23_{-0.10}^{+0.10}$ & $20.04_{-0.10}^{+0.10}$ & $16.99_{-0.40}^{+0.40}$ & $17.94_{-0.16}^{+0.11}$ & $15.28_{-0.06}^{+0.09}$ & $14.54_{-0.04}^{+0.05}$ & $<13.95$ & $5.4_{-0.7}^{+0.9}$ \\
\hline 64 & HD 101413 & $20.30_{-0.10}^{+0.10}$ & $20.08_{-0.10}^{+0.10}$ & $18.74_{-0.50}^{+0.50}$ & $\begin{array}{r}17.35_{-0.32}^{+0.30}\end{array}$ & $14.95_{-0.15}^{+0.15}$ & $14.32_{-0.08}^{+0.08}$ & $<14.22$ & $4.0_{-0.7}^{+1.0}$ \\
\hline 65 & HD $101436^{\mathrm{b}}$ & $20.34_{-0.05}^{+0.05}$ & $20.09_{-0.05}^{+0.05}$ & $18.53_{-0.50}^{+0.50}$ & $17.50_{-0.55}^{+0.70}$ & $15.51_{-0.10}^{+0.14}$ & $14.41_{-0.03}^{+0.03}$ & $<13.90$ & $\ldots$ \\
\hline & (blue) & $\ldots$ & $\ldots$ & $\ldots$ & $15.49_{-0.18}^{+1.80}$ & $14.29_{-0.54}^{+0.56}$ & -0.03 & -20 & $5.3^{+1.0}$ \\
\hline & (red) & $\ldots$ & $\ldots$ & $\ldots$ & $17.50^{+0.09}$ & $15.48^{+0.14}$ & $14.41^{+0.04}$ & $\ldots$ & $3.3^{+0.5}$ \\
\hline 66 & HD 103779 & $19.33_{-010}^{+0.10}$ & $19.65_{-010}^{+0.10}$ & $16.25_{-009}^{+0.15}$ & $15.83_{-0.07}^{+0.16}$ & $14.81_{-0.04}^{+0.10}$ & $14.75^{+0.10}+10$ & $<13.88$ & $13^{+0.9}$ \\
\hline 67 & HD $104705^{b}$ & $19.57_{-0.05}^{+0.05}$ & $19.77_{-0.05}^{+0.05}$ & $17.87_{-0.06}^{+0.09}$ & $17.28_{-0.12}^{+0.07}$ & $15.21_{-0.08}^{+0.04}$ & $14.46_{-0.03}^{+0.105}$ & $<13.95$ & $\ldots^{-1.0}$ \\
\hline & (blue) & -0.05 & -0.05 & $15.85_{-0.14}^{+0.06}$ & $15.30_{-0.08}^{+0.15}$ & $14.01_{-0.04}^{+0.04}$ & -0.03 & $\ldots$ & $7.1_{-1.0}^{+1.0}$ \\
\hline & (red) & $\ldots$ & $\ldots$ & $17.87_{-0.06}^{+0.08}$ & $17.28_{-0.13}^{+0.08}$ & $15.18_{-0.31}^{+0.03}$ & $14.46_{-0.05}^{+0.05}$ & $\ldots$ & $3.4_{-0}^{+0}$ \\
\hline 68 & HD 115071 & $20.30_{-0.10}^{+0.10}$ & $20.37_{-0.10}^{+0.10}$ & $18.30_{-0.22}^{+0.18}$ & $16.86_{-0.29}^{+0.38}$ & $15.55_{-0.08}^{+0.10}$ & $14.93_{-0.04}^{+0.05}$ & $<13.90$ & $10.2_{-1.0}^{+0.6}$ \\
\hline 69 & HD 116781 & $19.56_{-0.10}^{+0.10}$ & $19.89_{-0.10}^{+0.10}$ & $17.90_{-0.25}^{+0.19}$ & $17.55_{-0.37}^{+0.29}$ & $15.19_{-0.08}^{+0.11}$ & $14.61_{-0.04}^{+0.04}$ & $<14.00$ & $8.5_{-0.9}^{+0.10}$ \\
\hline 70 & HD 116852 & $19.50_{-0.05}^{+0.05}$ & $19.46_{-0.05}^{+0.05}$ & $15.99_{-0.20}^{+0.37}$ & $15.81_{-0.18}^{+0.32}$ & $14.64_{-0.04}^{-0.08}$ & $14.00_{-0.18}^{+0.04}$ & $<13.85$ & $\begin{array}{l}-1.0 \\
7.1_{-1.0}^{+1.0}\end{array}$ \\
\hline 71 & HD 118571 & $19.86_{-0.05}^{+0.05}$ & $20.03_{-0.05}^{+0.05}$ & $17.97_{-0.30}^{+0.16}$ & $17.65_{-0.39}^{+0.23}$ & $15.27_{-0.11}^{+0.04}$ & $14.50_{-0.04}^{+0.18}$ & $<14.04$ & $7.5_{-0.9}^{+1.0}$ \\
\hline 72 & HD $124314 \mathrm{~A}$ & $20.17_{-0.05}^{+0.05}$ & $20.16_{-0.05}^{+0.05}$ & $18.03_{-0.22}^{+0.30}$ & $16.99_{-0.34}^{+0.39}$ & $15.53_{-0.10}^{+0.13}$ & $14.75_{-0.04}^{+0.04}$ & $<13.64$ & $9.1_{-1}^{+0}$ \\
\hline 73 & HD 124979 & $20.02_{-0.10}^{+0.10}$ & $20.18_{-0.10}^{+0.10}$ & $18.21_{-0.20}^{+0.22}$ & $17.46_{-0.33}^{+0.32}$ & $15.51_{-0.08}^{+0.09}$ & $14.84_{-0.04}^{+0.04}$ & $<13.90$ & $10.0_{-1 .}^{+0.0}$ \\
\hline 74 & HD $148422^{\mathrm{c}}$ & $19.93_{-0.15}^{+0.15}$ & $19.70_{-0.15}^{+0.15}$ & $\ldots$ & $\ldots$ & $15.04_{-0.12}^{+0.12}$ & $14.30_{-0.20}^{+0.20}$ & $<14.30$ & $\ldots$ \\
\hline 75 & HD 152218 & $20.32_{-0.10}^{+0.10}$ & $20.20_{-0.10}^{+0.10}$ & $18.33_{-0.14}^{+0.11}$ & $17.50_{-0.42}^{+0.31}$ & $15.83_{-0.46}^{+0.12}$ & $15.14_{-0.09}^{+0.20}$ & $13.93_{-0.15}^{+0.15}$ & $7.0_{-1.0}^{+1.0}$ \\
\hline 76 & HD 152233 & $20.01_{-0.10}^{+0.10}$ & $19.95_{-0.10}^{+0.10}$ & $18.31_{-0.08}^{+0.06}$ & $18.11_{-0.10}^{+0.07}$ & $16.93_{-0.57}^{+0.40}$ & $15.39_{-0.21}^{-0.09}$ & $14.02_{-0.08}^{+0.08}$ & $4.3_{-0.9}^{-1.0}$ \\
\hline 77 & HD 152248 & $20.01_{-0.10}^{+0.10}$ & $19.95_{-0.10}^{+0.10}$ & $18.37_{-0.08}^{+0.08}$ & $18.14_{-0.14}^{+0.10}$ & $16.84_{-0.71}^{+0.51}$ & $15.36_{-0.44}^{+0.21}$ & $<13.90$ & $4.9_{-?}^{+c}$ \\
\hline 78 & HD $152314^{\mathrm{c}}$ & $20.27_{-0.10}^{+0.10}$ & $20.14_{-0.10}^{+0.10}$ & $\ldots=0.00$ & $\ldots$ & $15.70_{-0.18}^{+0.39}$ & $15.00_{-0.08}^{+0.08}$ & $<13.90$ & $7.0_{-1}^{+1}$ \\
\hline 79 & HD 152623 & $19.88_{-0.10}^{+0.10}$ & $19.93_{-0.10}^{+0.10}$ & $16.58_{-0.26}^{+0.33}$ & $16.34_{-0.23}^{+0.29}$ & $15.27_{-0.06}^{+0.08}$ & $14.84_{-0.04}^{+0.08}$ & $13.65_{-0.10}^{+0.10}$ & $10.4_{-10}^{+0.9}$ \\
\hline 80 & HD 152723 & $19.97_{-0.10}^{+0.10}$ & $19.99_{-0.10}^{+0.10}$ & $18.12_{-0.16}^{+0.20}$ & $17.83_{-0.26}^{+0.23}$ & $15.49_{-0.15}^{+0.30}$ & $14.84_{-0.06}^{+0.04}$ & $13.70_{-0.10}^{+0.10}$ & $5.9_{-0.9}^{+0.9}$ \\
\hline 81 & HD 153426 & $19.91_{-0.10}^{+0.10}$ & $20.07_{-0.10}^{+0.10}$ & $17.74_{-0.22}^{+0.18}$ & $17.11_{-0.40}^{+0.30}$ & $15.39_{-0.19}^{+0.69}$ & $14.86_{-0.09}^{+0.06}$ & $<13.81$ & $6.5_{-1.4}^{+0.9}$ \\
\hline 82 & HD $154368^{\mathrm{c}}$ & $21.02_{-0.15}^{+0.15}$ & $20.62_{-0.10}^{+0.10}$ & $\ldots{ }^{-0.22}$ & -0.40 & $15.41_{-0.18}^{+0.18}$ & $14.86_{-0.08}^{+0.09}$ & $\ldots$ & $\ldots^{-1.4}$ \\
\hline 83 & HD 156292 & $20.41_{-0.10}^{+0.10}$ & $20.58_{-0.10}^{+0.10}$ & $18.04_{-0.24}^{+0.21}$ & $16.56_{-0.28}^{+0.39}$ & $15.47_{-0.07}^{+0.10}$ & $14.88_{-0.05}^{+0.08}$ & $<13.78$ & $9.4_{-10}^{+0.9}$ \\
\hline 84 & HD 157857 & $20.31_{-0.10}^{+0.10}$ & $20.32_{-0.10}^{+0.10}$ & $18.50_{-0.16}^{+0.24}$ & $17.73_{-0.36}^{+0.28}$ & $15.36_{-0.09}^{+0.07}$ & $14.80_{-0.06}^{+0.05}$ & $<14.11$ & $7.1_{-}^{+}$ \\
\hline 85 & HD 158661 & $19.89_{-0.10}^{+0.10}$ & $19.83_{-0.10}^{+0.10}$ & $18.35_{-0.15}^{+0.16}$ & $17.75_{-0.29}^{+0.36}$ & $15.96_{-0.24}^{+0.09}$ & $15.51_{-0.19}^{+0.06}$ & $<14.10$ & $8.2_{-}^{+}$ \\
\hline 86 & HD 161807 & $19.35_{-0.05}^{+0.05}$ & $19.69_{-0.05}^{+0.05}$ & $17.73_{-0.17}^{+0.17}$ & $17.27_{-049}^{+0.30}$ & $15.00_{-0.08}^{+0.08}$ & $14.43_{-0.04}^{+0.04}$ & $<13.90$ & $6.4_{-0.8}^{+1.0}$ \\
\hline 87 & HD 163758 & $19.53_{-0.10}^{+0.10}$ & $19.56_{-0.10}^{+0.10}$ & $17.93_{-026}^{+0.19}$ & $18.06_{-0.25}^{-0.49}$ & $15.82_{-0.19}^{+0.31}$ & $15.31_{-0.06}^{+0.07}$ & $14.05_{-0.07}^{+0.07}$ & $9.0_{-}^{+}$ \\
\hline 88 & HD 163892 & $20.38_{-0.10}^{+0.10}$ & $20.14_{-0.10}^{+0.10}$ & $17.88_{-012}^{+0.12}$ & $17.26_{-0.30}^{+0.30}$ & $15.92_{-0.22}^{+0.48}$ & $15.43_{-0.09}^{+0.06}$ & $14.23_{-0.04}^{+0.07}$ & $5.2_{-1}^{+}$ \\
\hline 89 & HD 164816 & $19.69_{-0.10}^{+0.10}$ & $19.70_{-0.10}^{+0.10}$ & $17.55_{-0.09}^{+0.07}$ & $17.11_{-0.22}^{+0.16}$ & $14.93_{-0.14}^{+0.24}$ & $14.60_{-0.06}^{+0.09}$ & $<14.30$ & $2.8_{-6}^{+0}$ \\
\hline 90 & HD 165052 & $19.78_{-0.05}^{+0.05}$ & $19.99_{-0.05}^{+0.05}$ & $17.82_{-0.29}^{+0.19}$ & $17.43_{-0.39}^{+0.32}$ & $15.36_{-0.12}^{+0.16}$ & $14.59_{-0.04}^{+0.06}$ & $<14.00$ & $7.7_{-0.9}^{+0.4}$ \\
\hline 91 & HD 165246 & $19.83_{-0.10}^{+0.10}$ & $19.89_{-010}^{+0.10}$ & $15.59_{-0.08}^{+0.13}$ & $15.44_{-0.06}^{+0.10}$ & $14.66_{-0.05}^{+0.05}$ & $14.35_{-0.13}^{+0.04}$ & $<14.08$ & $8.7_{-10}^{+0.9}$ \\
\hline \multirow[t]{3}{*}{92} & HD $166546^{\mathrm{b}}$ & $19.99_{-0.10}^{+0.10}$ & $20.05_{-0.10}^{+0.10}$ & $\begin{array}{l}18.01_{-0.23}^{+0.08} \\
+0.19\end{array}$ & $17.54_{-0.37}^{+0.00}$ & $15.60_{-0.07}^{+0.06}$ & $15.17_{-0.03}^{+0.107}$ & $14.20_{-0.20}^{+0.10}$ & -1.0 \\
\hline & (blue) & $\ldots$ & $\ldots$ & 18.01 & 17.54 & 15.41 & 14.83 & $13.90_{-0.20}^{+0.10}$ & \\
\hline & (red) & $\ldots$ & $\ldots$ & 15.32 & 15.34 & 15.16 & 14.90 & $13.90_{-0.20}^{+0.10}$ & $\ldots$ \\
\hline
\end{tabular}


Table 3-Continued

\begin{tabular}{|c|c|c|c|c|c|c|c|c|c|}
\hline ID & Target & $\log \mathrm{N}(0)$ & $\log N(1)$ & $\log \mathrm{N}(2)$ & $\log \mathrm{N}(3)$ & $\log \mathrm{N}(4)$ & $\log \mathrm{N}(5)$ & $\log N(6)$ & $b\left(\mathrm{~km} \mathrm{~s}^{-1}\right)$ \\
\hline 93 & HD $166716^{c}$ & $19.95_{-0.10}^{+0.10}$ & $19.90_{-0.10}^{+0.10}$ & & & $15.65_{-0.13}^{+0.13}$ & $15.18_{-0.08}^{+0.08}$ & $<14.10$ & \\
\hline 94 & HD 167402 & $19.90_{-}^{+}$ & $19.74_{-0.10}^{+0.10}$ & $15.63_{-0.06}^{+0.04}$ & $15.42_{-0.06}^{+0.04}$ & $14.60_{-0.04}^{+0.13}$ & $14.37_{-0.08}^{+0.08}$ & $<14.00$ & $15.7_{-1.0}^{+2.5}$ \\
\hline \multirow[t]{3}{*}{95} & HD $167659^{b}$ & $20.13_{-0.10}^{+0.10}$ & $20.34_{-0.10}^{+0.10}$ & $17.59_{-0.37}^{+0.44}$ & $17.62_{-0.34}^{+0.27}$ & $16.01_{-0.11}^{+0.22}$ & $15.30_{-0.07}^{+0.15}$ & $13.98_{-0.10}^{+0.10}$ & \\
\hline & (blue) & $\ldots$ & $\ldots$ & $15.73_{-0.04}^{+0.07}$ & $15.48_{-0.09}^{+0.17}$ & $14.64_{-0.04}^{+0.04}$ & $14.28_{-0.04}^{+0.04}$ & $\ldots-0.10$ & $8.4^{+0.9}$ \\
\hline & (red) & $\ldots$ & $\ldots$ & $17.58_{-0.38}^{+0.44}$ & $17.62_{-0.34}^{+0.09}$ & $15.99_{-0.12}^{+0.04}$ & $15.26_{-0.08}^{+0.04}$ & . & $7.6_{-1.0}^{+0.0}$ \\
\hline 96 & HD 167771 & $20.39_{-0.05}^{+0.05}$ & $20.32_{-0.05}^{+0.05}$ & $19.05_{-0.50}^{+0.50}$ & $18.04_{-0.11}^{+0.07}$ & $15.50_{-0.06}^{+0.07}$ & $14.83_{-0.04}^{+0.05}$ & $<13.96$ & $6.1_{-0.6}^{+0.7}$ \\
\hline 97 & HD $167971^{\mathrm{c}}$ & $20.63_{-0.15}^{+0.15}$ & $20.49_{-0.10}^{+0.10}$ & $18.10_{-0.50}^{+0.50}$ & $16.20_{-0.30}^{+0.30}$ & $16.00_{-0.20}^{+0.30}$ & $<15.31$ & $\ldots$ & $15.0_{-2.0}^{+2.0}$ \\
\hline 98 & HD $168076^{c}$ & $20.45_{-0.15}^{+0.15}$ & $20.22_{-0.15}^{+0.15}$ & & & $16.50_{-0.20}^{+0.20}$ & $15.64_{-0.08}^{+0.08}$ & $<14.40$ & \\
\hline 99 & HD 168941 & $19.82_{-0.10}^{+0.10}$ & $19.77_{-0.10}^{+0.10}$ & $17.85_{-0.15}^{+0.13}$ & $17.29_{-0.46}^{+0.33}$ & $15.69_{-04}^{+1.1}$ & $14.64_{-0.41}^{+0.41}$ & $<14.08$ & $4.0_{-14}^{+1.0}$ \\
\hline 100 & HD 172140 & $18.97_{-0.10}^{+0.10}$ & $18.92_{-0.10}^{+0.10}$ & $15.78_{-0.29}^{+1.45}$ & $15.68_{-0.28}^{+1.48}$ & $14.62_{-0.0}^{+0.5}$ & $14.13_{-0.10}^{+0.10}$ & $<13.90$ & $5.1_{-2.0}^{+1.4}$ \\
\hline 101 & HD 175754 & $19.06_{-0.10}^{+0.10}$ & $19.36_{-0.10}^{+0.10}$ & $16.96_{-0.35}^{+0.43}$ & $16.57_{-0.27}^{+0.44}$ & $14.91_{-0.0}^{+0.0}$ & $14.30_{-0.04}^{+0.04}$ & $<13.95$ & $8.4_{-1.9}^{+2.9}$ \\
\hline \multirow[t]{3}{*}{102} & HD $175876^{\mathrm{b}}$ & $18.98_{-0.05}^{+0.05}$ & $19.30_{-0.05}^{+0.05}$ & $16.79_{-0.30}^{+0.35}$ & $16.46_{-0.13}^{+0.26}$ & $15.01_{-0.03}^{+0.05}$ & $14.58_{-0.03}^{+0.04}$ & $<14.06$ & $\ldots^{-1.0}$ \\
\hline & (blue) & $\ldots-0.00$ & $\ldots$ & 16.03 & $16.15^{-0.13}$ & $14.73^{-0.03}$ & $14.38^{-0.03}$ & $<13.75$ & $\ldots$ \\
\hline & (red) & & & 16.71 & 16.16 & 14.69 & 14.16 & $<13.75$ & \\
\hline 103 & HD 177989 & $19.93_{-0.10}^{+0.10}$ & $19.67_{-0.10}^{+0.10}$ & $17.64_{-0.20}^{+0.13}$ & $17.26_{-0.37}^{+0.25}$ & $15.39_{-0.17}^{+0.31}$ & $14.58_{-0.05}^{+0.06}$ & $<13.85$ & $4.4_{-0.6}^{+0.8}$ \\
\hline 104 & HD 178487 & $20.18_{-0.10}^{+0.10}$ & $20.16_{-0.10}^{+0.10}$ & $18.16_{-0.16}^{+0.11}$ & $17.75_{-0.28}^{+0.19}$ & $15.41_{-0.12}^{+0.20}$ & $14.66_{-0.10}^{+0.10}$ & $<14.06$ & $7.2_{-1.0}^{+1.0}$ \\
\hline 105 & HD 179406 & $20.41_{-0.10}^{+0.10}$ & $20.21_{-0.10}^{+0.10}$ & $18.09_{-0.14}^{+0.10}$ & $17.12_{-0.46}^{+0.55}$ & $15.25_{-0.10}^{+0.11}$ & $14.50_{-0.05}^{+0.09}$ & $<14.10$ & $5.5_{-1.5}^{+1.0}$ \\
\hline 106 & HD 179407 & $19.88_{-0.10}^{+0.10}$ & $19.94_{-0.10}^{+0.10}$ & $18.25_{-0.10}^{+0.10}$ & $17.98_{-0.18}^{+0.40}$ & $16.33_{-0.41}^{+0.93}$ & $14.83_{-0.08}^{+0.05}$ & $<14.00$ & $5.5_{-1.6}^{+1.0}$ \\
\hline 107 & HD 185418 & $20.23^{\top}$ & $20.53_{-0.10}^{+0.10}$ & $18.37_{-0.08}^{+0.07}$ & $17.43_{-0.41}^{+0.18}$ & $15.56_{-0}^{+1 .}$ & $14.53_{-0.07}^{+0.08}$ & $<13.90$ & $4.1_{-1.5}^{+0.9}$ \\
\hline 108 & HD 187459 & $20.12_{-0.10}^{+0.10}$ & $20.06_{-0.10}^{+0.10}$ & $17.56_{-0.34}^{+0.08}$ & $17.23_{-0.34}^{+0.31}$ & $15.41_{-0 .}^{+0 .}$ & $14.84_{-0.04}^{+0.05}$ & $<13.78$ & $9.7_{-1.0}^{+0.9}$ \\
\hline 109 & HD $190429 A$ & $19.89^{-}$ & $19.95_{-0}^{+0}$ & $16.39_{-0.40}^{+0.40}$ & $16.66_{-0.20}^{+0.27}$ & $15.57_{-0.07}^{+0.08}$ & $15.08_{-0.04}^{+0.04}$ & $13.99_{-0.15}^{+0.15}$ & $13.3_{-1.0}^{+0.9}$ \\
\hline 110 & HD 190918 & $19.31_{-0.10}^{+0.10}$ & $19.63_{-0.10}^{+0.10}$ & $16.56_{-0.27}^{+0.20}$ & $16.57_{-0.31}^{+0.20}$ & $15.39_{-0.06}^{+0.06}$ & $15.06_{-0.0}^{+0.0}$ & $13.76_{-0.15}^{+0.15}$ & $15.5_{-1.0}^{+2.4}$ \\
\hline 111 & HD 191495 & 19.68 & $19.85_{-0}^{+0}$ & $16.79_{-0.24}^{+0.32}$ & $16.67_{-0.24}^{+0.31}$ & $15.56_{-0.0}^{+0.1}$ & $15.05_{-0}^{+0}$ & $14.05_{-0.11}^{+0.15}$ & $11.8_{-1.0}^{+0.0}$ \\
\hline 112 & HD 191877 & $19.73_{-0}^{+0}$ & $19.70_{-0.10}^{+0.10}$ & $17.52_{-0.36}^{+0.17}$ & $17.06_{-0.4}^{+0.2}$ & $15.26_{-0.0}^{+0.0}$ & $14.75_{-0}^{+0}$ & $13.53_{-0.10}^{+0.11}$ & $6.1_{-0.6}^{+0.9}$ \\
\hline 113 & HD 192035 & $20.37_{-0}^{+0}$ & $20.35_{-0}^{+0}$ & $18.43_{-0.5}^{+0.5}$ & $16.69_{-0}^{+0}$ & $15.43_{-0}^{+0}$ & $14.67_{-0}^{+0}$ & $<14.08$ & $6.8_{-1}^{+1}$ \\
\hline 114 & HD 192639 & $20.27_{-0}^{+0}$ & $20.41_{-0}^{+0}$ & $18.28_{-0}^{+0}$ & $17.06_{-0}^{+0}$ & $15.64_{-0}^{+0}$ & $15.27_{-0}^{+0}$ & $14.08_{-0.11}^{+0.11}$ & $7.3_{-}^{+}$ \\
\hline 115 & HD 195965 & $19.90_{-0}^{+0}$ & $20.18_{-0.05}^{+0.05}$ & $18.09_{-0 .}^{+0.0}$ & $17.31_{-0.4}^{+0.2}$ & $15.43_{-0}^{+0}$ & $14.55_{-0}^{+0}$ & $<13.90$ & $6.4_{-0.7}^{+0.9}$ \\
\hline 116 & HD 199579 & $20.23_{-0.10}^{+0.10}$ & $20.19_{-0.10}^{+0.10}$ & $17.96_{-0.30}^{+0.13}$ & $17.38_{-0.27}^{+0.41}$ & $15.95_{-0}^{+0}$ & $15.47_{-0.05}^{+0.06}$ & $14.30_{-0.08}^{+0.08}$ & $13.2_{-1.0}^{+0.9}$ \\
\hline 117 & HD 201345 & $18.83_{-0.10}^{+0.10}$ & $19.02_{-0.10}^{+0.10}$ & $16.89_{-0.39}^{+0.52}$ & $16.49_{-0.27}^{+0.51}$ & $15.00_{-0}^{+0}$ & $14.57_{-0.04}^{+0.06}$ & $<13.60$ & $7.4_{-1.1}^{+0.9}$ \\
\hline 118 & HD 201638 & $17.67_{-0}^{+0 .}$ & $18.08_{-0}^{+0}$ & $16.09_{-0}^{+0}$ & $15.62_{-0}^{+0}$ & $14.30_{-0}^{+0}$ & $<14.30$ & $<14.30$ & $6.5_{-0.9}^{+0.1}$ \\
\hline 119 & HD 203374A & $20.32_{-0}^{+0}$ & $20.42_{-0}^{+0 .}$ & $18.09_{-0}^{+0}$ & $17.24_{-0}^{+0}$ & $15.22_{-0}^{+0}$ & $14.71_{-0.04}^{+0.04}$ & $<14.00$ & $8.0_{-}^{+\ell}$ \\
\hline 120 & HD 206267 & $20.65_{-0.10}^{+0.10}$ & $20.52_{-0.10}^{+0.10}$ & $18.17_{-0.20}^{+0.25}$ & $17.43_{-0.4}^{+0.6}$ & $15.93_{-0}^{+1}$ & $15.21_{-0.14}^{+1.10}$ & $14.03_{-0.11}^{+0.11}$ & $5.7_{-2.4}^{+1.0}$ \\
\hline 121 & HD 206773 & $20.05_{-0.05}^{+0.05}$ & $20.22_{-0.05}^{+0.05}$ & $18.10_{-0.12}^{+0.07}$ & $17.35_{-0.33}^{+0.18}$ & $15.44_{-0}^{+0}$ & $14.70_{-0.03}^{+0.04}$ & $13.62_{-0.15}^{+0.15}$ & $5.7_{-0.5}^{+0.7}$ \\
\hline 122 & HD 207198 & $20.61_{-0}^{+0}$ & $20.43_{-0.10}^{+0.10}$ & $18.42_{-0.08}^{+0.06}$ & $17.63_{-0.29}^{+0.20}$ & $16.65_{-0}^{+0}$ & $16.68_{-0.17}^{+0.41}$ & $14.04_{-0.11}^{+0.11}$ & $3.8_{-}^{+1}$ \\
\hline 123 & HD 207308 & $20.52_{-0}^{+0}$ & $20.38_{-0.10}^{+0.10}$ & $18.28_{-0.13}^{+0.13}$ & $17.03_{-0.55}^{+0.73}$ & $15.35_{-0}^{+0}$ & $14.77_{-0.05}^{+0.11}$ & $<14.30$ & $5.2_{-}^{+}$ \\
\hline 124 & HD 208440 & $19.94_{-0.05}^{+0.05}$ & $20.00_{-0.05}^{+0.05}$ & $17.73_{-0}^{+0}$ & $17.26_{-0}^{+0}$ & $15.37_{-0}^{+0}$ & $14.91_{-0}^{+0}$ & $<14.30$ & $8.1_{-1}^{+1} \quad r$ \\
\hline 125 & HD 209339 & $19.80_{-0.05}^{+0.05}$ & $19.97_{-0.05}^{+0.05}$ & $17.19_{-0}^{+0}$ & $16.44_{-0.37}^{+0.54}$ & $15.15_{-0}^{+0}$ & $14.69_{-0}^{+0}$ & $<14.10$ & $6.8_{-}^{+}-x-$ \\
\hline \multirow[t]{3}{*}{126} & HD $210809^{\mathrm{b}}$ & $19.54_{-0.05}^{+0.05}$ & $19.74_{-0.05}^{+0.05}$ & $16.36_{-0.40}^{+0.40}$ & $16.46_{-0.40}^{+0.40}$ & $15.56_{-0.03}^{+0.03}$ & $15.09_{-0.03}^{+0.03}$ & $14.15_{-0.06}^{+0.06}$ & $5.0_{-1.0}^{+1.0}$ \\
\hline & (blue) & $\ldots$ & $\ldots$ & $\ldots$ & $\ldots$ & $14.72_{-0.04}^{+0.06}$ & $14.33_{-0.04}^{+0.04}$ & 0.0 .00 & $\cdots$ \\
\hline & (red) & $\ldots$ & $\ldots$ & $\ldots$ & $\ldots$ & $15.50_{-c}^{+c}$ & $15.01_{-c}^{+c}$ & $\ldots$ & \\
\hline 127 & HD 210839 & $20.51_{-0.10}^{+0.10}$ & $20.45_{-0.10}^{+0.10}$ & $18.06_{-0.43}^{+0.45}$ & $16.85_{-0.22}^{+0.08}$ & $15.78_{-c}^{+C}$ & $15.30_{-0}^{+0}$ & $14.31_{-0.06}^{+0.06}$ & $10.0_{-1.0}^{+1.0}$ \\
\hline 128 & HD 216044 & $19.75_{-}^{+}$ & $20.00_{-0}^{+0}$ & $17.66_{-0.31}^{+0.26}$ & $17.36_{-0.35}^{+0.35}$ & $15.56_{-}^{+}$ & $15.15_{-0}^{+0}$ & $13.99_{-}^{+}$ & $8.7_{-1.0}^{+0.9}$ \\
\hline 129 & HD $216532^{\mathrm{c}}$ & $20.85_{-0}^{+0 .}$ & $20.74_{-0.15}^{+0.15}$ & $\ldots$ & $\ldots$ & $15.78_{-0}^{+0}$ & $15.48_{-0.08}^{+0.18}$ & $14.46_{-0.08}^{+0.08}$ & \\
\hline 130 & HD 216898 & $20.66_{-0.10}^{+0.10}$ & $20.78_{-0.10}^{+0.10}$ & $18.77_{-0.36}^{+0.36}$ & $17.69_{-0.31}^{+0.28}$ & $16.03_{-0}^{+0}$. & $15.52_{-0.06}^{+0.08}$ & $14.20_{-0.11}^{+0.11}$ & $9.8_{-1.0}^{+0.9}$ \\
\hline 131 & HD 217305 & $20.65_{-0.10}^{+0.10}$ & $20.61_{-0}^{+0}$ & $18.93_{-0}^{+0}$ & $17.67_{-0}^{+0}$ & $16.19_{-c}^{+c}$ & $15.46_{-0.07}^{+0.00}$ & $14.29_{-0.10}^{+0.11}$ & \\
\hline 132 & HD 217312 & $20.43_{-0}^{+0}$ & $20.54_{-0}^{+0}$ & $18.44_{-0.14}^{+0.13}$ & $17.26_{-0}^{+0}$ & $15.91_{-}^{+}$ & $15.27_{-0.05}^{+0.06}$ & $14.16_{-0.08}^{+0.08}$ & $9.4_{-1.0}^{+0.9}$ \\
\hline 133 & HD 218915 & $19.78_{-0}^{+0 .}$ & $19.94_{-0.10}^{+0.10}$ & $17.95_{-0.30}^{+0.18}$ & $17.50_{-0}^{+0}$ & $15.54_{-0.10}^{+0.11}$ & $14.87_{-0.05}^{+0.05}$ & $13.83_{-0.10}^{+0.08}$ & $8.2_{-0.9}^{+0.9}$ \\
\hline \multirow[t]{3}{*}{134} & HD $224151^{\mathrm{b}}$ & $20.12_{-0.10}^{+0.10}$ & $20.32_{-0.10}^{+0.10}$ & $18.15_{-0.22}^{+0.13}$ & $17.66_{-0.35}^{+0.22}$ & $15.66_{-0.16}^{+0.30}$ & $14.94_{-0.03}^{+0.03}$ & $14.06_{-0.16}^{+0.16}$ & $\cdots$ \\
\hline & (blue) & $\ldots$ & $\ldots$ & $15.69_{-}^{+c}$ & $15.36_{-0}^{+0}$ & $14.59_{-}^{+}$ & $14.32_{-0.04}^{+0.03}$ & $13.84_{-0.15}^{+0.10}$ & $e_{0}+2$ \\
\hline & (red) & $\ldots$ & $\ldots$ & $18.15_{-0.22}^{+0.13}$ & $17.66_{-0.35}^{+0.22}$ & $15.62_{-0.19}^{+0.34}$ & $14.82_{-0.04}^{+0.09}$ & $13.64_{-0.18}^{+0.18}$ & $\cdots$ \\
\hline \multirow[t]{3}{*}{135} & HD $224257^{\mathrm{b}}$ & $19.44_{-0.10}^{+0.10}$ & $19.81_{-0.10}^{+0.10}$ & $16.22_{-0.20}^{+0.44}$ & $15.94_{-0.19}^{+0.41}$ & $14.94_{-0.03}^{+0.03}$ & $14.77_{-0.03}^{+0.04}$ & $<14.20$ & - \\
\hline & (blue) & $\ldots$ & $\ldots$ & $16.12_{-0.30}^{+0.48}$ & $15.83_{-0.29}^{+0.45}$ & $14.46_{-}^{+}$ & $14.31_{-0.04}^{+0.04}$ & $<13.90$ & $6.4_{-1.0}^{+1.0}$ \\
\hline & (red) & $\ldots$ & $\ldots$ & $15.53_{-0.04}^{+0.04}$ & $15.28_{-0.04}^{+0.04}$ & $14.76_{-0.04}^{+0.04}$ & $14.59_{-0.04}^{+0.04}$ & $<13.90$ & $19.5_{-1.0}^{+0.5}$ \\
\hline 136 & HD 224868 & $20.02_{-0.10}^{+0.10}$ & $20.18_{-0.10}^{+0.10}$ & $17.09_{-0.33}^{+0.41}$ & $16.56_{-0.26}^{+0.37}$ & $15.11_{-0.06}^{+0.08}$ & $14.63_{-0.13}^{+0.13}$ & $<13.95$ & $10.0_{-1.0}^{+0.9}$ \\
\hline 137 & HD $303308^{b}$ & $19.69_{-0.10}^{+0.10}$ & $20.08_{-0.10}^{+0.10}$ & $17.14_{-0.40}^{+0.40}$ & $16.95_{-0.40}^{+0.40}$ & $16.50_{-0.22}^{+0.34}$ & $16.67_{-0.24}^{+0.35}$ & $15.71_{-0.09}^{+0.08}$ & \\
\hline
\end{tabular}


Table 3-Continued

\begin{tabular}{clllllllll}
\hline \hline ID & Target & $\log \mathrm{N}(0)$ & $\log \mathrm{N}(1)$ & $\log \mathrm{N}(2)$ & $\log \mathrm{N}(3)$ & $\log \mathrm{N}(4)$ & $\log \mathrm{N}(5)$ & $\log \mathrm{N}(6)$ & $b(\mathrm{~km} \mathrm{~s})$ \\
\hline & (blue) & $\ldots$ & $\ldots$ & $\ldots$ & $\ldots$ & $16.39_{-0.32}^{+0.40}$ & $16.63_{-0.28}^{+0.37}$ & $15.55_{-0.08}^{+0.10}$ & $7.7_{-0.9}^{+0.9}$ \\
& (red) & $\ldots$ & $\ldots$ & $\ldots$ & $\ldots$ & $15.86_{-0.12}^{+0.19}$ & $15.63_{-0.04}^{+0.07}$ & $15.21_{-0.04}^{+0.04}$ & $11.5_{-1.0}^{+1.0}$ \\
138 & HD 308813 & $19.97_{-0.10}^{+0.10}$ & $20.00_{-0.10}^{+0.10}$ & $18.05_{-0.17}^{+0.16}$ & $17.42_{-0.36}^{+0.31}$ & $15.07_{-0.06}^{+0.13}$ & $14.48_{-0.13}^{+0.13}$ & $<13.95$ & $7.8_{-1.0}^{+0.9}$ \\
139 & HD 332407 & $20.03_{-0.10}^{+0.10}$ & $20.12_{-0.10}^{+0.10}$ & $16.80_{-0.40}^{+0.40}$ & $16.20_{-0.14}^{+0.22}$ & $15.45_{-0.06}^{+0.07}$ & $15.10_{-0.04}^{+0.05}$ & $14.20_{-0.08}^{+0.08}$ & $12.7_{-1.0}^{+0.9}$ \\
\hline
\end{tabular}

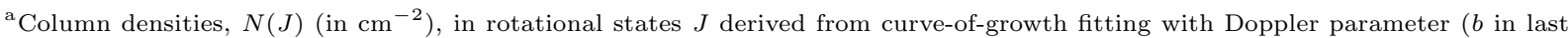
column) and damping wings for lines from $J=0$ and $J=1$. No value is listed when $b$ was poorly determined. Error bars on low- $J$ states $(J=0,1$ and sometimes $J=2,3)$ depend on data quality (see footnote in Table 3). In damping-wing fits, errors on log $N$ typically range from \pm 0.03 to \pm 0.10 . Six targets had detectable column densities in $J=7$ : \#48 with $\log N(7)=15.54 \pm 0.15$, \#52 with $\log N(7)=14.47 \pm 0.05, \# 88$ with $\log N(7)=14.14 \pm 0.06$, \#116 with $\log N(7)=14.29 \pm 0.11, \# 127$ with $\log N(7)=14.25 \pm 0.05$, and \#137 with $\log N(7)=15.43 \pm 0.15$ (15.32 in blue component and 14.80 in red component).

${ }^{\mathrm{b}}$ In 15 sight lines, multiple velocity components were observable and measured in high- $J$ states. We report individual column densities, denoted as "blue and red" components, and sum them to find total column densities.

${ }^{\mathrm{c}} \mathrm{A}$ few targets have no listed column densities $N(2)$ or $N(3)$. In several cases this was a result of poor data quality, so that lines from $J \geq 2$ could not be fitted accurately. In other cases, the accessible (unblocked) lines from $J=2$ and $J=3$ were highly saturated, with equivalent widths of 200-300 $\mathrm{m} \AA$ and large effective Doppler parameters $\left(b \geq 10 \mathrm{~km} \mathrm{~s}^{-1}\right)$ likely produced by unresolved velocity components. When equivalent widths of all the accessible lines lie on the "flat portion" of the curve of growth, errors can exceed \pm 0.40 for $\log N=16-17$ and \pm 0.50 for $\log N=17-19$. 
Table 4. Column Densities ${ }^{\mathrm{a}}$ and Rotational Temperatures ${ }^{\mathrm{b}}$

\begin{tabular}{|c|c|c|c|c|c|c|c|c|c|c|c|c|}
\hline ID & Target & $\log N_{\mathrm{H} 2}$ & $\begin{array}{l}\mathrm{T}_{01} \\
(\mathrm{~K})\end{array}$ & $\begin{array}{l}\mathrm{T}_{02} \\
(\mathrm{~K})\end{array}$ & $\begin{array}{l}\mathrm{T}_{24} \\
(\mathrm{~K})\end{array}$ & $\begin{array}{l}\mathrm{T}_{35} \\
(\mathrm{~K})\end{array}$ & $\log N_{\mathrm{HI}}{ }^{\mathrm{c}}$ & $\log N_{\mathrm{H}}{ }^{\mathrm{c}}$ & $\begin{array}{l}D_{\text {phot }}{ }^{\mathrm{d}} \\
(\mathrm{kpc})\end{array}$ & $\begin{array}{c}\left\langle n_{\mathrm{H}}\right\rangle^{\mathrm{d}} \\
\left(\mathrm{cm}^{-3}\right)\end{array}$ & $f_{\mathrm{H} 2}{ }^{\mathrm{d}}$ & Notes $^{a}$ \\
\hline 1 & $\mathrm{BD} 35^{\circ} 4258$ & 19.56 & 125 & 125 & 222 & 247 & 21.24 & 21.26 & 2.51 & 0.22 & 0.040 & 2 \\
\hline 2 & $\mathrm{BD} 53^{\circ} 2820$ & 20.04 & 107 & 52 & 473 & 590 & 21.35 & 21.39 & 4.27 & 0.17 & 0.089 & 2 \\
\hline 3 & $\mathrm{CPD}-59^{\circ} 2600$ & 20.16 & 81 & 57 & 274 & 381 & 21.54 & 21.57 & 2.11 & 0.57 & 0.077 & 2 \\
\hline 4 & $\mathrm{CPD}-59^{\circ} 2603$ & 20.13 & 87 & 99 & 141 & 595 & 21.43 & 21.47 & 2.60 & 0.37 & 0.091 & 1 \\
\hline 5 & $\mathrm{CPD}-69^{\circ} 1743$ & 19.91 & 101 & 50 & 376 & 491 & 21.16 & 21.21 & 4.47 & 0.36 & 0.101 & 2 \\
\hline 6 & $\mathrm{CPD}-72^{\circ} 1184$ & 20.07 & 115 & 73 & 173 & & 20.90 & 21.01 & 9.85 & 0.034 & 0.228 & 2 \\
\hline 7 & HD 3827 & 17.48 & 159 & 159 & 274 & 348 & 20.55 & 20.55 & 1.88 & 0.061 & 0.0017 & 1 \\
\hline 8 & HD 5005A & 20.22 & 86 & 92 & 172 & 277 & 21.40 & 21.45 & 2.89 & 1.03 & 0.117 & 1 \\
\hline 9 & HD 12323 & 20.31 & 93 & 85 & 181 & 236 & 21.19 & 21.29 & 3.04 & 0.21 & 0.209 & 2 \\
\hline 10 & HD 13268 & 20.47 & 111 & 85 & 181 & 288 & 21.34 & 21.44 & 2.80 & 0.32 & 0.212 & 2 \\
\hline 11 & HD 13745 & 20.59 & 75 & 98 & 156 & 260 & 21.34 & 21.47 & 2.81 & 0.34 & 0.262 & 2 \\
\hline 12 & HD 14434 & 20.54 & 117 & 123 & 141 & 303 & 21.45 & 21.55 & 2.98 & 0.39 & 0.197 & 2 \\
\hline 13 & HD 15137 & 20.27 & 119 & 76 & 215 & 274 & 21.24 & 21.32 & 3.00 & 0.23 & 0.176 & 1 \\
\hline 14 & HD $15558 \mathrm{~A}$ & 20.89 & 93 & 103 & 142 & 290 & 21.52 & 21.69 & 1.91 & 0.83 & 0.319 & 3 \\
\hline 15 & HD 15642 & 20.33 & 159 & 331 & 118 & 187 & 21.23 & 21.33 & 3.89 & 0.18 & 0.201 & 2 \\
\hline 16 & HD 34656 & 20.19 & 94 & 66 & 209 & 276 & $21.22^{*}$ & 21.29 & 1.99 & 0.32 & 0.157 & 1 \\
\hline 17 & HD 39680 & 19.53 & 90 & 73 & 148 & 273 & 21.30 & 21.31 & 2.67 & 0.25 & 0.033 & 1 \\
\hline 18 & HD 41161 & 20.02 & 96 & 81 & 178 & 221 & 21.01 & 21.09 & 1.16 & 0.34 & 0.170 & 3 \\
\hline 19 & HD 42088 & 20.51 & 82 & 71 & 190 & 257 & 21.15 & 21.31 & 1.97 & 0.34 & 0.314 & 1 \\
\hline 20 & HD 45314 & 20.60 & 89 & 77 & 158 & 204 & 21.04 & 21.28 & 0.80 & 0.77 & 0.421 & 1 \\
\hline 21 & HD 46150 & 20.64 & 97 & 55 & 273 & 383 & 21.26 & 21.43 & 1.47 & 0.59 & 0.324 & 1 \\
\hline 22 & HD 47360 & 20.38 & 88 & 74 & 192 & 329 & 21.46 & 21.53 & 1.24 & 0.89 & 0.143 & 2 \\
\hline 23 & HD 47417 & 20.52 & 89 & 71 & 188 & 243 & 21.13 & 21.30 & 1.23 & 0.53 & 0.329 & 1 \\
\hline 24 & HD 60369 & 20.48 & 67 & 74 & 187 & 219 & 21.26 & 21.38 & 2.54 & 0.31 & 0.249 & 1 \\
\hline 25 & HD 61347 & 20.60 & 81 & 99 & 145 & 237 & 21.53 & 21.62 & 4.44 & 0.30 & 0.190 & 2 \\
\hline 26 & HD 62866 & 20.24 & 104 & 58 & 329 & 426 & $21.23^{*}$ & 21.31 & 3.51 & 0.19 & 0.170 & 2 \\
\hline 27 & HD 63005 & 20.15 & 111 & 84 & 184 & 244 & 21.24 & 21.31 & 4.35 & 0.15 & 0.140 & 2 \\
\hline 28 & HD 64568 & 20.54 & 61 & 51 & 321 & 370 & $21.16^{*}$ & 21.33 & 5.75 & 0.12 & 0.324 & 2 \\
\hline 29 & HD 66695 & 20.11 & 75 & 96 & 208 & 277 & $21.12^{*}$ & 21.20 & 4.25 & 0.12 & 0.163 & 2 \\
\hline 30 & HD 66788 & 19.72 & 86 & 136 & 159 & 171 & 21.23 & 21.26 & 4.72 & 0.12 & 0.058 & 1 \\
\hline 31 & HD 69106 & 19.73 & 80 & 95 & 192 & 193 & 21.07 & 21.11 & 1.10 & 0.38 & 0.084 & 2 \\
\hline 32 & HD 73882 & 21.13 & 53 & 44 & 293 & 676 & 21.11 & 21.60 & 0.83 & 1.55 & 0.677 & 3 \\
\hline 33 & HD 74194 & 20.55 & 97 & 79 & 178 & 257 & $21.23^{*}$ & 21.38 & 2.25 & 0.35 & 0.295 & 2 \\
\hline 34 & HD 74920 & 20.26 & 117 & 55 & 306 & 390 & 21.15 & 21.25 & 2.05 & 0.28 & 0.205 & 1 \\
\hline 35 & HD 89137 & 19.99 & 110 & 54 & 304 & 400 & 21.03 & 21.10 & 4.04 & 0.10 & 0.154 & 1 \\
\hline 36 & HD 90087 & 19.91 & 92 & 83 & 206 & 284 & 21.19 & 21.23 & 2.52 & 0.22 & 0.095 & 1 \\
\hline 37 & HD 91597 & 19.70 & 69 & 56 & 378 & 463 & 21.40 & 21.42 & 4.29 & 0.20 & 0.038 & 2 \\
\hline 38 & HD 91651 & 19.07 & 82 & 72 & 412 & 400 & 21.15 & 21.16 & 3.98 & 0.12 & 0.016 & 1 \\
\hline 39 & HD 91824 & 19.83 & 65 & 86 & 186 & 237 & 21.12 & 21.22 & 2.75 & 0.20 & 0.204 & 1 \\
\hline 40 & HD 92554 & 18.93 & 40 & 61 & 419 & 519 & 21.34 & 21.34 & 6.61 & 0.11 & 0.0077 & 2 \\
\hline 41 & HD 93028 & 19.55 & 111 & 58 & 336 & 379 & 20.95 & 20.98 & 2.97 & 0.10 & 0.074 & 2 \\
\hline 42 & HD 93129A & 20.21 & 129 & 76 & 254 & 238 & 21.47 & 21.52 & 2.21 & 0.49 & 0.099 & 2 \\
\hline 43 & HD 93146A & 19.71 & 82 & 54 & 355 & 457 & 21.18 & 21.21 & 2.30 & 0.23 & 0.063 & 2 \\
\hline 44 & HD 93204 & 19.81 & 105 & 56 & 338 & 413 & 21.41 & 21.43 & 2.73 & 0.32 & 0.048 & 1 \\
\hline 45 & HD 93205 & 19.79 & 110 & 62 & 282 & 341 & 21.36 & 21.38 & 2.44 & 0.32 & 0.051 & 1 \\
\hline 46 & HD 93206 & 19.52 & 84 & 55 & 309 & 345 & 21.34 & 21.35 & 1.48 & 0.49 & 0.029 & 1 \\
\hline 47 & HD 93222 & 19.77 & 74 & 50 & 378 & 450 & 21.47 & 21.49 & 2.47 & 0.41 & 0.038 & 1 \\
\hline 48 & HD 93250 & 20.27 & 115 & 54 & 582 & 656 & 21.39 & 21.45 & 2.20 & 0.41 & 0.132 & 1 \\
\hline 49 & HD 93843 & 19.61 & 111 & 58 & 313 & 390 & 21.30 & 21.32 & 2.85 & 0.24 & 0.039 & 1 \\
\hline 50 & HD 96670 & 20.59 & 71 & 90 & 166 & 341 & $21.28^{*}$ & 21.43 & 2.67 & 0.33 & 0.290 & 2 \\
\hline 51 & HD 96715 & 20.62 & 57 & 85 & 163 & 256 & 21.20 & 21.38 & 3.15 & 0.25 & 0.345 & 2 \\
\hline 52 & HD 96917 & 20.25 & 64 & $\ldots$ & $\ldots$ & $\ldots$ & 21.23 & 21.31 & 2.46 & 0.27 & 0.173 & 2 \\
\hline 53 & HD 97471 & 19.90 & 67 & 69 & 245 & 299 & $21.20^{*}$ & 21.24 & 2.78 & 0.20 & 0.091 & 2 \\
\hline 54 & HD 97913 & 20.13 & 90 & 63 & 269 & 329 & $21.20^{*}$ & 21.27 & 2.52 & 0.24 & 0.145 & 2 \\
\hline
\end{tabular}


Table 4-Continued

\begin{tabular}{|c|c|c|c|c|c|c|c|c|c|c|c|c|}
\hline ID & Target & $\log N_{\mathrm{H} 2}$ & $\begin{array}{l}\mathrm{T}_{01} \\
(\mathrm{~K})\end{array}$ & $\begin{array}{l}\mathrm{T}_{02} \\
(\mathrm{~K})\end{array}$ & $\begin{array}{l}\mathrm{T}_{24} \\
(\mathrm{~K})\end{array}$ & $\begin{array}{l}\mathrm{T}_{35} \\
(\mathrm{~K})\end{array}$ & $\log N_{\mathrm{HI}}{ }^{\mathrm{c}}$ & $\log N_{\mathrm{H}}{ }^{\mathrm{c}}$ & $\begin{array}{l}D_{\text {phot }}{ }^{\mathrm{d}} \\
(\mathrm{kpc})\end{array}$ & $\begin{array}{c}\left\langle n_{\mathrm{H}}\right\rangle^{\mathrm{d}} \\
\left(\mathrm{cm}^{-3}\right)\end{array}$ & $f_{\mathrm{H} 2}{ }^{\mathrm{d}}$ & Notes $^{\mathrm{a}}$ \\
\hline 55 & HD 99857 & 20.26 & 88 & 92 & 150 & 184 & 21.27 & 21.35 & 3.04 & 0.24 & 0.163 & 2 \\
\hline 56 & HD 99890 & 19.47 & 91 & 75 & 244 & 282 & 21.12 & 21.14 & 3.22 & 0.14 & 0.043 & 2 \\
\hline 57 & HD 100199 & 20.17 & 98 & 65 & 198 & 253 & 21.18 & 21.26 & 2.77 & 0.21 & 0.163 & 2 \\
\hline 58 & HD 100213 & 20.42 & 79 & 86 & 162 & 205 & 21.18 & 21.33 & 2.15 & 0.32 & 0.285 & 2 \\
\hline 59 & HD 100276 & 19.83 & 85 & 59 & 313 & 554 & 21.19 & 21.23 & 2.94 & 0.19 & 0.080 & 2 \\
\hline 60 & HD 101131 & 20.28 & 87 & 88 & 164 & 196 & $21.15^{*}$ & 21.25 & 1.78 & 0.32 & 0.212 & 1 \\
\hline 61 & HD 101190 & 20.42 & 64 & 86 & 149 & 181 & 21.15 & 21.35 & 2.09 & 0.35 & 0.232 & 1 \\
\hline 62 & HD 101205 & 20.26 & 79 & 103 & 155 & 181 & 21.24 & 21.29 & 1.64 & 0.39 & 0.187 & 3 \\
\hline 63 & HD 101298 & 20.45 & 65 & 56 & 259 & 180 & 21.26 & 21.28 & 2.58 & 0.24 & 0.237 & 2 \\
\hline 64 & HD 101413 & 20.51 & 63 & 98 & 143 & 200 & 21.23 & 21.37 & 2.13 & 0.36 & 0.276 & 2 \\
\hline 65 & HD 101436 & 20.54 & 61 & 88 & 155 & 197 & 21.23 & 21.28 & 1.85 & 0.33 & 0.290 & 1 \\
\hline 66 & HD 103779 & 19.82 & 117 & 59 & 300 & 507 & 21.17 & 21.21 & 3.99 & 0.13 & 0.082 & 2 \\
\hline 67 & HD 104705 & 19.99 & 98 & 92 & 175 & 214 & 21.15 & 21.21 & 4.18 & 0.13 & 0.122 & 1 \\
\hline 68 & HD 115071 & 20.64 & 84 & 82 & 254 & 304 & 21.39 & 21.42 & 1.87 & 0.46 & 0.262 & 2 \\
\hline 69 & HD 116781 & 20.06 & 119 & 94 & 172 & 206 & 21.21 & 21.27 & 1.79 & 0.34 & 0.124 & 2 \\
\hline 70 & HD 116852 & 19.78 & 74 & 53 & 317 & 322 & 20.96 & 21.01 & 4.88 & 0.068 & 0.117 & 1 \\
\hline 71 & HD 118571 & 20.44 & 94 & 86 & 172 & 193 & $20.98^{*}$ & 21.18 & 2.70 & 0.18 & 0.366 & 1 \\
\hline 72 & HD $124314 \mathrm{~A}$ & 20.47 & 77 & 78 & 185 & 265 & 21.41 & 21.50 & 1.25 & 0.82 & 0.187 & 1 \\
\hline 73 & HD 124979 & 20.41 & 93 & 88 & 172 & 230 & 21.27 & 21.30 & 3.09 & 0.21 & 0.258 & 2 \\
\hline 74 & HD 148422 & 20.13 & 63 & $\ldots$ & $\ldots$ & & 21.24 & 21.30 & 8.26 & 0.078 & 0.134 & 3 \\
\hline 75 & HD 152218 & 20.57 & 69 & 82 & 185 & 253 & 21.34 & 21.47 & 1.42 & 0.67 & 0.254 & 2 \\
\hline 76 & HD 152233 & 20.29 & 73 & 92 & 311 & 222 & 21.29 & 21.37 & 1.52 & 0.50 & 0.167 & 2 \\
\hline 77 & HD 152248 & 20.29 & 73 & 95 & 285 & 217 & $21.27^{*}$ & 21.44 & 1.61 & 0.55 & 0.143 & 2 \\
\hline 78 & HD 152314 & 20.51 & 68 & $\ldots$ & $\ldots$ & $\ldots$ & $21.35^{*}$ & 21.46 & 1.44 & 0.65 & 0.224 & 2 \\
\hline 79 & HD 152623 & 20.21 & 82 & 55 & 325 & 381 & 21.28 & 21.35 & 1.04 & 0.70 & 0.145 & 2 \\
\hline 80 & HD 152723 & 20.29 & 79 & 87 & 176 & 203 & 21.43 & 21.49 & 1.94 & 0.52 & 0.127 & 2 \\
\hline 81 & HD 153426 & 20.30 & 93 & 77 & 195 & 264 & 21.34 & 21.41 & 1.83 & 0.46 & 0.154 & 2 \\
\hline 82 & HD 154368 & 21.17 & 55 & $\ldots$ & $\ldots$ & $\ldots$ & 21.00 & 21.60 & 1.08 & 1.20 & 0.747 & 3 \\
\hline 83 & HD 156292 & 20.81 & 94 & 72 & 180 & 345 & $21.29^{*}$ & 21.51 & 1.51 & 0.69 & 0.398 & 2 \\
\hline 84 & HD 157857 & 20.62 & 78 & 88 & 150 & 207 & 21.30 & 21.45 & 2.56 & 0.36 & 0.295 & 2 \\
\hline 85 & HD 158661 & 20.17 & 73 & 99 & 192 & 265 & $21.28^{*}$ & 21.34 & 4.08 & 0.17 & 0.134 & 2 \\
\hline 86 & HD 161807 & 19.86 & 121 & 95 & 171 & 213 & $21.08^{*}$ & 21.13 & 1.94 & 0.23 & 0.108 & 1 \\
\hline 87 & HD 163758 & 19.86 & 80 & 96 & 215 & 219 & 21.23 & 21.27 & 4.11 & 0.15 & 0.079 & 2 \\
\hline 88 & HD 163892 & 20.58 & 62 & 69 & 230 & 319 & 21.32 & 21.46 & 1.37 & 0.68 & 0.267 & 2 \\
\hline 89 & HD 164816 & 20.00 & 78 & 78 & 177 & 239 & 21.18 & 21.23 & 1.08 & 0.51 & 0.117 & 2 \\
\hline 90 & HD 165052 & 20.20 & 99 & 83 & 187 & 213 & 21.36 & 21.42 & 1.37 & 0.62 & 0.122 & 1 \\
\hline 91 & HD 165246 & 20.16 & 83 & 45 & 429 & 503 & 21.41 & 21.46 & 1.38 & 0.68 & 0.101 & 2 \\
\hline 92 & HD 166546 & 20.32 & 83 & 82 & 191 & 252 & $21.19^{*}$ & 21.29 & 1.46 & 0.43 & 0.212 & 2 \\
\hline 93 & HD 166716 & 20.23 & 74 & $\ldots$ & $\ldots$ & & $21.27^{*}$ & 21.34 & 2.73 & 0.26 & 0.154 & 2 \\
\hline 94 & HD 167402 & 20.13 & 66 & 45 & 396 & 519 & 21.13 & 21.21 & 7.61 & 0.069 & 0.167 & 2 \\
\hline 95 & HD 167659 & 20.55 & 99 & 68 & 277 & 257 & 21.30 & 21.43 & 1.87 & 0.47 & 0.262 & 2 \\
\hline 96 & HD 167771 & 20.67 & 72 & 109 & 134 & 190 & 21.08 & 21.33 & 1.40 & 0.49 & 0.438 & 1 \\
\hline 97 & HD 167971 & 20.87 & 68 & 69 & 152 & $\ldots$ & 21.60 & 21.74 & 1.42 & 1.25 & 0.271 & 3 \\
\hline 98 & HD 168076 & 20.65 & 63 & $\ldots$ & $\ldots$ & $\ldots$ & 21.65 & 21.73 & 1.97 & 0.88 & 0.167 & 3 \\
\hline 99 & HD 168941 & 20.10 & 74 & 83 & 211 & 227 & 21.18 & 21.25 & 3.72 & 0.15 & 0.143 & 2 \\
\hline 100 & HD 172140 & 19.25 & 74 & 57 & 360 & 370 & 21.11 & 21.12 & 6.54 & 0.065 & 0.027 & 2 \\
\hline 101 & HD 175754 & 19.54 & 113 & 79 & 221 & 262 & 21.04 & 21.07 & 2.55 & 0.15 & 0.059 & 2 \\
\hline 102 & HD 175876 & 19.47 & 117 & 77 & 250 & 311 & 21.04 & 21.06 & 2.57 & 0.14 & 0.051 & 1 \\
\hline 103 & HD 177989 & 20.12 & 61 & 74 & 203 & 225 & 20.99 & 21.09 & 5.14 & 0.078 & 0.202 & 2 \\
\hline 104 & HD 178487 & 20.47 & 76 & 81 & 169 & 197 & 21.22 & 21.35 & 5.22 & 0.14 & 0.262 & 2 \\
\hline 105 & HD 179406 & 20.62 & 64 & 73 & 164 & 230 & 21.23 & 21.40 & 0.21 & 3.88 & 0.329 & 2 \\
\hline 106 & HD 179407 & 20.22 & 83 & 95 & 234 & 193 & 21.20 & 21.28 & 7.72 & 0.080 & 0.173 & 2 \\
\hline 107 & HD 185418 & 20.71 & 113 & 87 & 166 & 209 & 21.19 & 21.41 & 0.78 & 1.07 & 0.398 & 2 \\
\hline 108 & HD 187459 & 20.39 & 73 & 68 & 212 & 250 & $21.31^{*}$ & 21.40 & 1.68 & 0.48 & 0.194 & 2 \\
\hline
\end{tabular}


Table 4 - Continued

\begin{tabular}{|c|c|c|c|c|c|c|c|c|c|c|c|c|}
\hline ID & Target & $\log N_{\mathrm{H} 2}$ & $\begin{array}{l}\mathrm{T}_{01} \\
(\mathrm{~K})\end{array}$ & $\begin{array}{l}\mathrm{T}_{02} \\
(\mathrm{~K})\end{array}$ & $\begin{array}{l}\mathrm{T}_{24} \\
(\mathrm{~K})\end{array}$ & $\begin{array}{l}\mathrm{T}_{35} \\
(\mathrm{~K})\end{array}$ & $\log N_{\mathrm{HI}}{ }^{\mathrm{c}}$ & $\log N_{\mathrm{H}}{ }^{\mathrm{c}}$ & $\begin{array}{l}D_{\text {phot }}{ }^{\mathrm{d}} \\
(\mathrm{kpc})\end{array}$ & $\begin{array}{c}\left\langle n_{\mathrm{H}}\right\rangle^{\mathrm{d}} \\
\left(\mathrm{cm}^{-3}\right)\end{array}$ & $f_{\mathrm{H} 2}{ }^{\mathrm{d}}$ & Notes $^{a}$ \\
\hline 109 & HD 190429A & 20.22 & 83 & 53 & 473 & 364 & $21.33^{*}$ & 21.39 & 2.38 & 0.33 & 0.134 & 2 \\
\hline 110 & HD 190918 & 19.80 & 117 & 64 & 357 & 379 & 21.40 & 21.42 & 2.39 & 0.36 & 0.048 & 2 \\
\hline 111 & HD 191495 & 20.07 & 94 & 62 & 343 & 356 & $21.32^{*}$ & 21.37 & 1.69 & 0.45 & 0.101 & 2 \\
\hline 112 & HD 191877 & 20.02 & 75 & 76 & 202 & 258 & 21.03 & 21.11 & 2.07 & 0.20 & 0.163 & 2 \\
\hline 113 & HD 192035 & 20.66 & 76 & 84 & 156 & 292 & 21.20 & 21.40 & 2.29 & 0.36 & 0.366 & 2 \\
\hline 114 & HD 192639 & 20.65 & 91 & 82 & 176 & 326 & 21.32 & 21.47 & 2.14 & 0.45 & 0.300 & 2 \\
\hline 115 & HD 195965 & 20.37 & 110 & 88 & 175 & 219 & 20.92 & 21.11 & 1.03 & 0.41 & 0.360 & 1 \\
\hline 116 & HD 199579 & 20.51 & 74 & 75 & 225 & 307 & 21.04 & 21.24 & 0.92 & 0.61 & 0.371 & 2 \\
\hline 117 & HD 201345 & 19.24 & 97 & 84 & 237 & 306 & 21.00 & 21.01 & 2.50 & 0.13 & 0.034 & 2 \\
\hline 118 & HD 201638 & 18.23 & 136 & 97 & 249 & $\ldots$ & $20.80^{*}$ & 20.80 & 8.98 & 0.023 & 0.0054 & 2 \\
\hline 119 & HD $203374 \mathrm{~A}$ & 20.68 & 87 & 76 & 163 & 237 & 21.20 & 21.41 & 0.78 & 1.07 & 0.377 & 2 \\
\hline 120 & HD 206267 & 20.89 & 68 & 70 & 204 & 268 & 21.22 & 21.51 & 0.73 & 1.44 & 0.483 & 2 \\
\hline 121 & HD 206773 & 20.45 & 94 & 84 & 175 & 227 & 21.09 & 21.24 & 0.69 & 0.82 & 0.314 & 1 \\
\hline 122 & HD 207198 & 20.83 & 65 & 77 & 251 & 564 & 21.28 & 21.51 & 1.04 & 1.01 & 0.415 & 2 \\
\hline 123 & HD 207308 & 20.76 & 68 & 75 & 160 & 263 & 21.20 & 21.44 & 0.76 & 1.17 & 0.421 & 2 \\
\hline 124 & HD 208440 & 20.27 & 83 & 76 & 195 & 254 & 21.24 & 21.32 & 1.04 & 0.65 & 0.176 & 1 \\
\hline 125 & HD 209339 & 20.19 & 94 & 67 & 222 & 332 & 21.20 & 21.28 & 1.08 & 0.57 & 0.163 & 1 \\
\hline 126 & HD 210809 & 19.95 & 98 & 57 & 482 & 413 & 21.31 & 21.35 & 3.88 & 0.18 & 0.080 & 1 \\
\hline 127 & HD 210839 & 20.78 & 73 & 70 & 201 & 370 & 21.24 & 21.47 & 0.87 & 1.21 & 0.409 & 2 \\
\hline 128 & HD 216044 & 20.20 & 105 & 79 & 216 & 269 & $21.28^{*}$ & 21.35 & 2.57 & 0.28 & 0.123 & 2 \\
\hline 129 & HD 216532 & 21.10 & 70 & $\ldots$ & & & $21.38^{*}$ & 21.69 & 0.94 & 1.69 & 0.512 & 3 \\
\hline 130 & HD 216898 & 21.03 & 89 & 86 & 170 & 273 & $21.44^{*}$ & 21.65 & 0.91 & 1.59 & 0.438 & 2 \\
\hline 131 & HD 217035 & 20.94 & 74 & 92 & 170 & 269 & 21.46 & 21.67 & 0.72 & 2.10 & 0.377 & 2 \\
\hline 132 & HD 217312 & 20.79 & 88 & 82 & 183 & 296 & 21.48 & 21.63 & 0.60 & 2.30 & 0.290 & 2 \\
\hline 133 & HD 218915 & 20.17 & 93 & 88 & 191 & 229 & 21.20 & 21.27 & 3.62 & 0.17 & 0.157 & 2 \\
\hline 134 & HD 224151 & 20.53 & 98 & 83 & 185 & 222 & 21.35 & 21.46 & 0.91 & 1.03 & 0.232 & 2 \\
\hline 135 & HD 224257 & 19.96 & 127 & 57 & 332 & 473 & $21.08^{*}$ & 21.14 & 2.05 & 0.22 & 0.132 & 2 \\
\hline 136 & HD 224868 & 20.41 & 93 & 61 & 228 & 304 & $21.16^{*}$ & 21.29 & 3.03 & 0.21 & 0.262 & 2 \\
\hline 137 & HD 303308 & 20.23 & 131 & 68 & 569 & 1358 & 21.41 & 21.46 & 2.66 & 0.35 & 0.117 & 2 \\
\hline 138 & HD 308813 & 20.29 & 80 & 85 & 157 & 206 & 21.20 & 21.30 & 3.45 & 0.19 & 0.197 & 2 \\
\hline 139 & HD 332407 & 20.38 & 86 & 56 & 317 & 499 & 21.24 & 21.35 & 2.56 & 0.28 & 0.216 & 2 \\
\hline
\end{tabular}

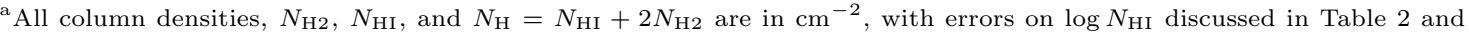
Section 3.2. Values of $N(0)$ and $N(1)$ come from fitting damping-wing Voigt profiles of $\mathrm{R}(0), \mathrm{R}(1)$, and $\mathrm{P}(1)$ lines. Their errors depend on data quality. Notes in last column refer to three levels of $\mathrm{S} / \mathrm{N}$ ratio: (1) $S / N \geq 15$ with errors of $\pm 0.03-0.05$ on $\log N ;(2) 5 \leq S / N \leq 15$ with errors of $\pm 0.05-0.10$; (3) $S / N \leq 5$, with errors of $\pm 0.10-0.20$.

${ }^{\mathrm{b}}$ The rotational excitation temperature $T_{01}$ is determined from the ratio $N_{1} / N_{0}$ of column densities of the $J=1$ and $J=0$ rotational states. Similar excitation temperatures $T_{02}, T_{24}$ and $T_{35}$ follow from ratios $N_{2} / N_{0}, N_{4} / N_{2}$, and $N_{5} / N_{3}$ of populations in levels $J=0,2,4$ (para- $\left.\mathrm{H}_{2}\right)$ and $J=3,5$ (ortho- $\left.\mathrm{H}_{2}\right)$ respectively, as described in Sections 3.4 and 3.5.

${ }^{\mathrm{c}}$ Neutral hydrogen column densities $N_{\mathrm{HI}}$ are taken from previous surveys by Diplas \& Savage (1994), Jenkins (2019), Fitzpatrick \& Massa (1990), and several individual papers. Details are discussed in Section 2.1 and Table 2. For 26 sight lines with no Ly $\alpha$ fits, labeled with asterisks after $\log N_{\mathrm{HI}}$, we use the relation, $N_{\mathrm{H}}=\left(5.8 \times 10^{21} \mathrm{~cm}^{-2} \mathrm{mag}^{-1}\right) E(B-V)$ from the Copernicus survey (Savage et al. 1977), with $N_{\mathrm{HI}}=N_{\mathrm{H}}-2 N_{\mathrm{H} 2}$. Updated values of color excess $E(B-V)$ for all 139 target stars are listed in Table 1, taken from Shull \& Danforth (2019).

${ }^{\mathrm{d}}$ Mean total hydrogen density along each sight line, $\left\langle n_{\mathrm{H}}\right\rangle=N_{\mathrm{H}} / D_{\mathrm{phot}}$, is estimated from stellar photometric distances $D_{\text {phot }}$ in Table 1. The molecular fraction is defined by $f_{\mathrm{H} 2}=2 N_{\mathrm{H} 2} /\left[N_{\mathrm{HI}}+2 N_{\mathrm{H} 2}\right]$. 
Table 5. Observational Averages from FUSE Survey ${ }^{\mathrm{a}}$

\begin{tabular}{llccc}
\hline \hline \multicolumn{1}{c}{ Quantity } & \multicolumn{1}{c}{ Units } & $D \leq 2 \mathrm{kpc}$ & $D \leq 5 \mathrm{kpc}$ & All \\
\hline Sample & \# Stars & 56 & 129 & 138 \\
$\langle D\rangle$ & $\mathrm{kpc}$ & 1.31 & 2.27 & 2.62 \\
$\left\langle n_{\mathrm{HI}}\right\rangle$ & $\mathrm{cm}^{-3}$ & 0.553 & 0.364 & 0.344 \\
$\left\langle n_{\mathrm{H} 2}\right\rangle$ & $\mathrm{cm}^{-3}$ & 0.261 & 0.136 & 0.129 \\
$\left\langle n_{\mathrm{H}}\right\rangle$ & $\mathrm{cm}^{-3}$ & 0.814 & 0.500 & 0.472 \\
$\left\langle f_{\mathrm{H} 2}\right\rangle$ & $\ldots$ & 0.268 & 0.202 & 0.200 \\
$\langle E(B-V) / D\rangle$ & $\mathrm{mag} \mathrm{kpc}^{-1}$ & 0.434 & 0.260 & 0.246 \\
$\left\langle N_{\mathrm{H}} / E(B-V)\right\rangle$ & $\mathrm{cm}^{-2} \mathrm{mag}^{-1}$ & $6.00 \times 10^{21}$ & $6.06 \times 10^{21}$ & $6.07 \times 10^{21}$ \\
\hline
\end{tabular}

${ }^{a}$ Mean values of total hydrogen density, $n_{\mathrm{H}}=N_{\mathrm{H}} / D_{\text {phot }}$, and molecular fraction $f_{\mathrm{H} 2}$ along the sightlines for stars in the full FUSE survey and for sub-samples within two photometric distance limits (Shull \& Danforth 2019). The averages are column density-weighted (see Section 3.1). Column 3 is for 56 stars with $D_{\text {phot }} \leq 2 \mathrm{kpc}$, Column 4 is for 129 stars with $D_{\text {phot }} \leq 5 \mathrm{kpc}$, and Column 5 is for the full sample, omitting HD 3827 (uncertain $E(B-V)$. Statistics for $N_{H} / E(B-V)$ omit 26 stars without Ly $\alpha$ profile fits for H I. 